\title{
Prenatal Lactation-Focused Motivational Interviewing for Enhancing Breastfeeding Initiation, Exclusivity, and Duration: Feasibility and Preliminary Outcomes
}

\author{
Sarah H. Addicks
}

Follow this and additional works at: https://researchrepository.wvu.edu/etd

\author{
Recommended Citation \\ Addicks, Sarah H., "Prenatal Lactation-Focused Motivational Interviewing for Enhancing Breastfeeding \\ Initiation, Exclusivity, and Duration: Feasibility and Preliminary Outcomes" (2018). Graduate Theses, \\ Dissertations, and Problem Reports. 5028. \\ https://researchrepository.wvu.edu/etd/5028
}

This Dissertation is protected by copyright and/or related rights. It has been brought to you by the The Research Repository @ WVU with permission from the rights-holder(s). You are free to use this Dissertation in any way that is permitted by the copyright and related rights legislation that applies to your use. For other uses you must obtain permission from the rights-holder(s) directly, unless additional rights are indicated by a Creative Commons license in the record and/ or on the work itself. This Dissertation has been accepted for inclusion in WVU Graduate Theses, Dissertations, and Problem Reports collection by an authorized administrator of The Research Repository @ WVU.

For more information, please contact researchrepository@mail.wvu.edu. 
Prenatal Lactation-Focused Motivational Interviewing for Enhancing Breastfeeding Initiation, Exclusivity, and Duration: Feasibility and Preliminary Outcomes

Sarah H. Addicks, MS, MPH

Dissertation submitted to the Eberly College of Arts and Sciences

at West Virginia University

in partial fulfillment of the requirements for the degree of

Doctor of Philosophy

in

Psychology

Daniel W. McNeil, PhD, Chair

Melissa D. Blank, PhD

Christina L. Duncan, $\mathrm{PhD}$

Robert Duval, $\mathrm{PhD}$

Kevin T. Larkin, $\mathrm{PhD}$

Department of Psychology

Morgantown, West Virginia

2018

Keywords: breastfeeding, Motivational Interviewing, lactation, theory of planned behavior Copyright 2018 Sarah H. Addicks, MS, MPH 


\begin{abstract}
Prenatal Lactation-Focused Motivational Interviewing for Enhancing Breastfeeding Initiation, Exclusivity, and Duration: Feasibility and Preliminary Outcomes
\end{abstract}

Sarah H. Addicks, MS, MPH

Breastfeeding promotes health and well-being for both mother and infant. A variety of environmental and individual factors, including psychological ones, affect infant feeding practices. The purpose of this study was to determine the feasibility and effectiveness of a single-session Motivational Interviewing (MI) intervention delivered during the third trimester of pregnancy for enhancing breastfeeding outcomes. The Theory of Planned Behavior provided a theoretical framework for the intervention and outcome measures. The sample consisted of predominantly rural participants living in the North Central Appalachian region. Women were recruited through social media, local clinics, and word of mouth. Participants $(N=81)$ completed one in-person session during the third trimester of pregnancy and one follow-up phone call at one month postpartum. All participants were randomly assigned to either the MI intervention or a psychoeducation intervention focused on infant developmental milestones. Prepost intervention outcome measures included infant feeding intentions, perceived behavioral control of breastfeeding, perceived importance of breastfeeding, infant feeding knowledge, breastfeeding attitudes and subjective norms, and knowledge of infant development. At one month postpartum, participants completed a phone interview which assessed breastfeeding initiation and current breastfeeding status. Directly following the intervention, there was a significant effect of the MI intervention on increasing breastfeeding attitudes among primiparous women only $(p<.05)$. In addition, at one month postpartum, women in the MI group were more likely to report any current breastfeeding than women in the psychoeducation group, $\chi^{2}(1, N=$ $79)=4.30, p=0.040, \Phi=.233$. There were no significant between-group differences on intentions, perceived behavioral control, perceived importance, subjective norms, infant feeding knowledge, knowledge of infant development, likelihood of exclusive breastfeeding at one month postpartum, total proportion of feedings that were breast milk at one month postpartum, or plans for continued breastfeeding at one month postpartum. Results of this study support the feasibility of a single-session, prenatal MI intervention. Preliminary findings demonstrate MI's effectiveness in increasing the likelihood of any breastfeeding at one month postpartum, and in enhancing breastfeeding attitudes among primiparous women. Future work in this area may benefit from implementing electronic communication to reinforce messages of MI interventions. From a public health perspective, future work in this area should target populations facing breastfeeding inequities and disparities. This trial was registered on clinicaltrials.gov NCT03033459 


\section{Acknowledgements}

This project would not have been possible without the 81 women who invited me into their hearts and (quite literally) their homes to participate in this project. It was a joy and privilege to know each of them. I owe countless thanks to my mentor and dissertation chair, Dr. Dan McNeil, whom I am honored to call a role model, colleague, and friend. Your endless optimism, patience, and warmth have been invaluable to my development as a clinical psychologist and human being. Thank you also to my committee members-Drs. Melissa Blank, Christina Duncan, Robert Duval, Kevin Larkin, and Kimberly Foley-your expertise and feedback made this project the best it could be. Chelsey Morgan was a "godsend" to study completion; she is a stellar clinician and loyal friend. Deja Clement, Carlie Enns, Morgan Simpson, Miriam Flatley, and Kaleigh Westfall were essential to recruitment, video coding, and data entry. Thanks to the WVU Departments of Family Medicine and Obstetrics and Gynecology, and Morgantown WIC for help with recruitment.

I am grateful to my APP Laboratory labmates for their support. Special thanks to Cameron Randall and Alison Vargovich, for their love, solidarity, and reminders that graduate school is a "marathon and not a sprint." My friendships with you two are the greatest gifts that graduate school has given me. To my parents, David and Sharonne, thank you for telling me that I “could” every time I said I "couldn't," and always nurturing my academic and creative pursuits. I will always want to make you proud, and I love you. To my brother, Drew, thank you for always tolerating my "Type A-ness” with humor and pragmatism. I love you. Lastly, to my husband, Ben-you have been a patient and loving force throughout my training. I am lucky to have someone so special in my life with whom I can celebrate each joy and milestone. I love you. 
This project was generously supported by the WVU Foundation Distinguished Doctoral

Scholarship, the WVU Eberly College of Arts and Science Dissertation Research Award, and the WVU Department of Psychology Dissertation Research Award. 
Table of Contents

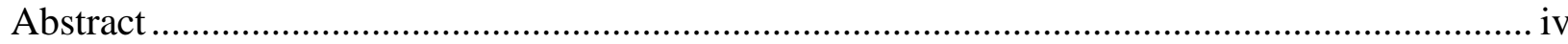

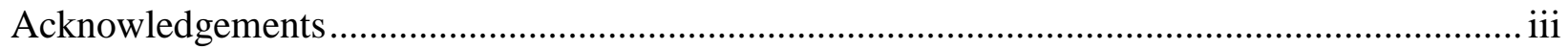

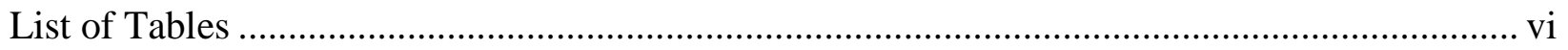

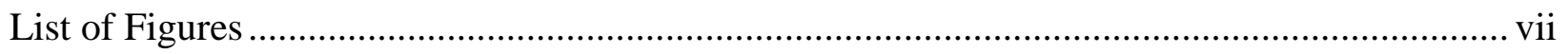

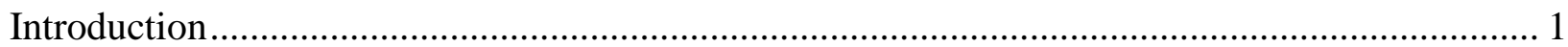

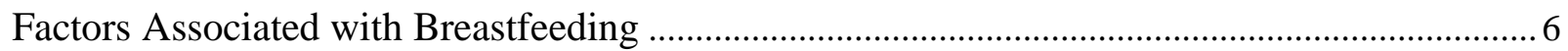

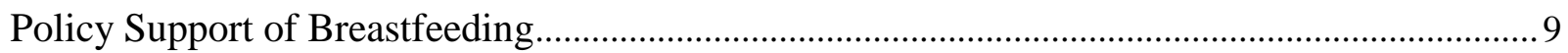

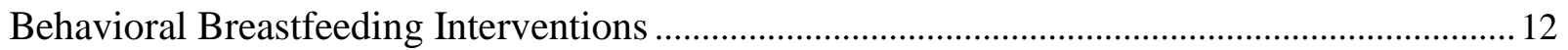

Theory of Planned Behavior and Breastfeeding .................................................................... 14

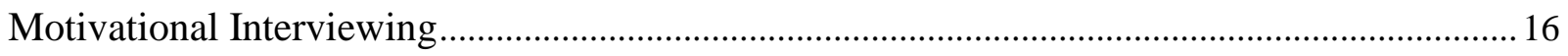

Rationale for Motivational Interviewing During Pregnancy ..................................................24

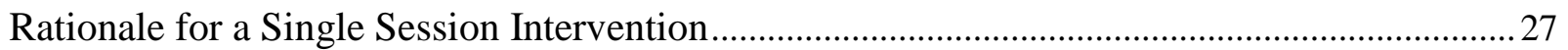

Rationale for a Rural, Appalachian Target Population .............................................................2 28

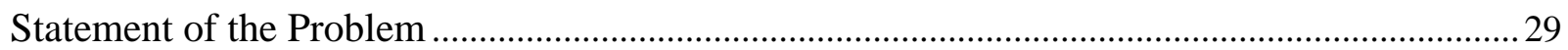

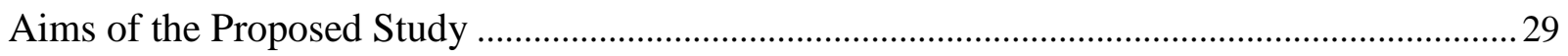

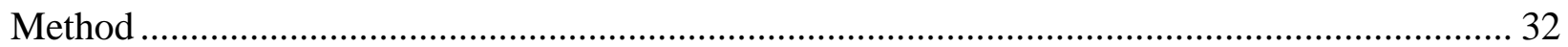

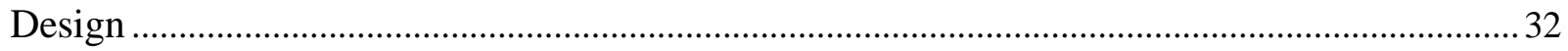

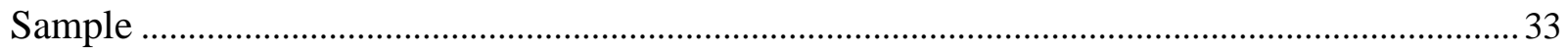

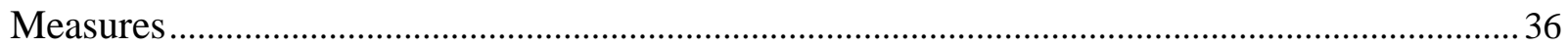

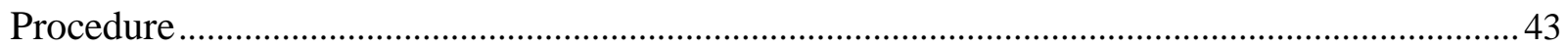

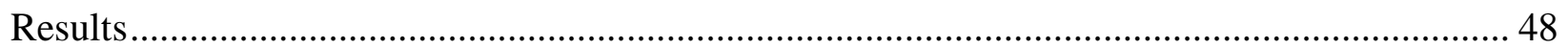

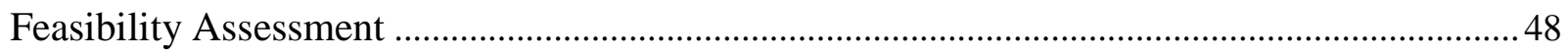

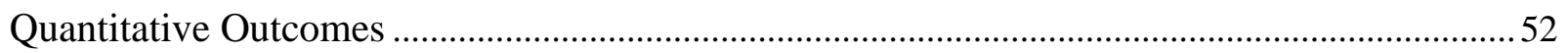

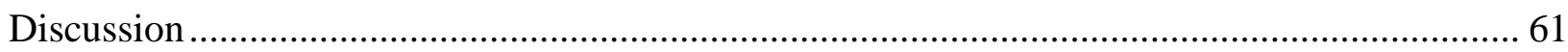

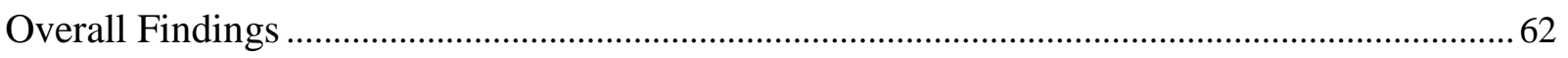

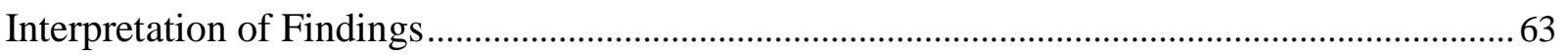

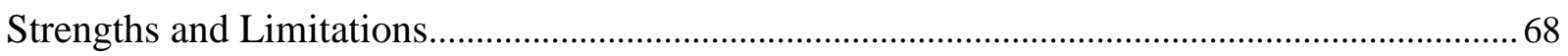

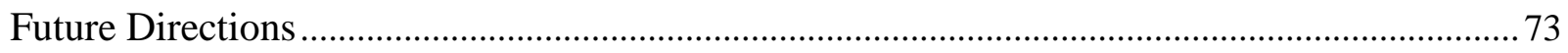

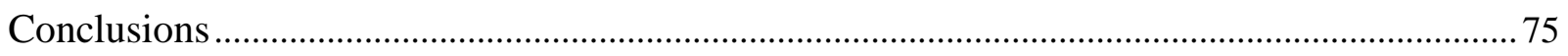

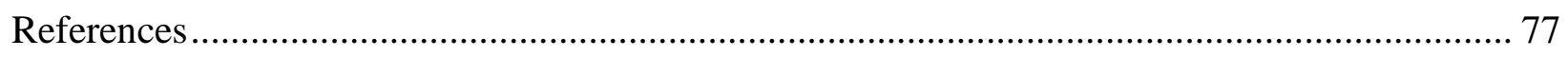

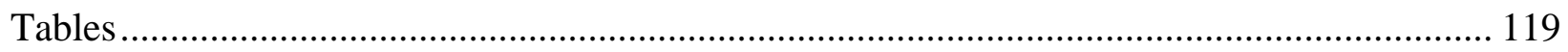

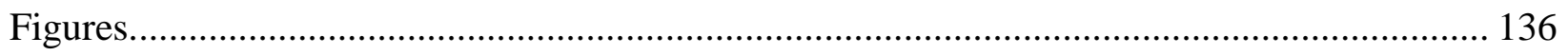

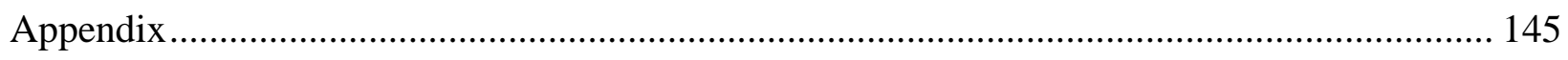


List of Tables

1. Interagency Group for Action on Breastfeeding Categories and Corresponding Items in the Postpartum Interview

2. Recruitment and In-Person Session Information

3. Participant Residence Location

4. Summary of Missing Data per Participant

5. Skewness and Kurtosis of Dependent Variables

6. Correlations of Primary Dependent Variable Instruments

7. Baseline Demographic Characteristics

8. Baseline Breastfeeding-Related Experiences and Perceptions Variables

9. Baseline Health and Mental Health Variables

10. One Month Postpartum Baby and Birth Variables

11. Baseline Dependent Variables

12. One Month Postpartum Feeding Characteristics 


\section{List of Figures}

1. CONSORT diagram

2. Mean ( \pm 1 SEM) intended breastfeeding duration in months at pre- and post-intervention for Motivational Interviewing and psychoeducation groups.

3. Mean ( $\pm 1 \mathrm{SEM})$ perceived behavioral control of breastfeeding at pre- and postintervention for Motivational Interviewing and psychoeducation groups.

4. Mean $( \pm 1 \mathrm{SEM})$ perceived breastfeeding importance at pre- and post-intervention for Motivational Interviewing and psychoeducation groups.

5. Mean ( \pm 1 SEM) Infant Feeding Knowledge Form (IFKF) scores at pre- and postintervention for Motivational Interviewing and psychoeducation groups.

6. Mean ( \pm 1 SEM) Iowa Infant Feeding Attitudes Scale (IIFAS) scores at pre- and postintervention for Motivational Interviewing and psychoeducation groups.

7. Mean ( \pm 1 SEM) Iowa Infant Feeding Attitudes Scale (IIFAS) scores at pre- and postintervention for Motivational Interviewing and psychoeducation groups by parity status.

8. Mean ( \pm 1 SEM) Brief Breastfeeding Attitudes Scale (BBAS) scores at pre- and postintervention for Motivational Interviewing and psychoeducation groups.

9. Mean ( \pm 1 SEM) Knowledge of Infant Development Inventory (KIDI) scores at pre- and post-intervention for Motivational Interviewing and psychoeducation groups. 
Prenatal Lactation-Focused Motivational Interviewing for Enhancing Breastfeeding Initiation, Exclusivity, and Duration: Feasibility and Preliminary Outcomes

Breastfeeding, or the practice of feeding human milk to an infant or child, promotes health for both the infant and mother (Ip, Chung, Raman, Trikalinos, \& Lau, 2009; Victora et al., 2016). Human milk is considered the optimal source of nutrition for most infants (Riordan \& Wambach, 2010). A complex substance made up of fat, carbohydrates, protein, water, vitamins, and minerals, breast milk has immunological and bioactive properties that promote health and development (Riordan \& Wambach, 2010). Breast milk contains white blood cells, whey proteins, oligosaccharides, and secretory immunoglobulin A which benefit the immune system and help prevent infection (Hanson, 2004). Breast milk also contains substances that aid in digestion and the development of the digestive tract (Sheard, 1988). Accordingly, breastfeeding is associated with a decreased risk of many conditions and diseases, including sudden infant death syndrome (SIDS), ear and lower respiratory infections, diabetes, overeating, obesity, sleep disorders, and childhood behavioral disorders (Bartick \& Reinhold, 2010; Brew et al., 2014; Kramer et al., 2008; McNiel, Labbok, \& Abrahams, 2010; Reynolds, Hennessy, \& Polek, 2014; Saltzman, Cole, Bost, Fiese, \& Donovan, 2017; Vennemann et al., 2009; Vinha \& de MelloFilho, 2017). Breastfeeding also may help to promote maternal-child bonding (Else-Quest, Hyde, \& Clark, 2003; Jackson, 2016; Jansen, Weerth, \& Riksen-Walraven, 2008).

Women who breastfeed experience less bleeding after delivery; reduced risk of several diseases and groups, including ovarian and breast cancers, hypertension, myocardial infarction, and diabetes; and faster return to pre-pregnancy weight than those who do not breastfeed (Bartick et al., 2013; Ip et al., 2009; Schwarz et al., 2009; Stuebe et al., 2009; Stuebe et al., 2011; Stuebe \& Schwarz, 2010; Unar-Munguí, Torres-Mejía, \& Colchero, 2017; Victora et al., 2016). 
These maternal-child risks are dose-responsive, such that increased duration of breastfeeding progressively reduces risk (Stuebe et al., 2009; Unar-Munguí et al., 2017).

Taken together, these findings suggest that breastfeeding promotes health and prevents disease in both infant and mother, and decreases overall population disease burden (Bartick et al., 2017; Grieken et al., 2013; Stuebe, 2009). As such, most major health-promoting organizations recommend breast milk as the singular source of infant nutrition for the first six months of life, and complementary provision of breast milk for one to two years or longer depending upon the needs and desires of the mother and baby (American Academy of Family Practice Breastfeeding Advisory Committee, 2014; American Academy of Pediatrics Section on Breastfeeding, 2012; World Health Organization [WHO], 2016). Actual rates of exclusive breastfeeding fall far short of this recommendation. Worldwide, $37 \%$ of infants receive exclusive breast milk for six months (Victora et al., 2016). Most women in the United States initiate breastfeeding, but only about one-fifth continue exclusively for six months (Centers for Disease Control and Prevention [CDC], 2016). It has been estimated that the current rate of "suboptimal" breastfeeding in the United States (defined by Bartick et al., 2017, as < 90\% of women breastfeeding for less than one year) is associated with increased pediatric and maternal morbidity and mortality (e.g., breast cancer, hypertension, SIDS) and substantial economic cost from premature death and healthcare expenses (Bartick et al., 2010; Bartick et al., 2013; Bartick et al., 2017).

There are many reasons that women choose not to breastfeed or to discontinue breastfeeding (Odom, Li, Scanlon, Perrine, \& Grummer-Strawn, 2013; Scott, Binns, Oddy, \& Graham, 2006). The top reasons for not breastfeeding or discontinuing breastfeeding in the United States include fatigue, inconvenience, return to work or school, and perceived problem 
with milk supply (Brown, Dodds, Legge, Bryanton, \& Semenic, 2014; Zimmerman \& Guttman, 2000). Many women report breast and nipple pain associated with breastfeeding, as well as chapped, dry, and bleeding skin around the nipple, which can negatively impact breastfeeding outcomes (Jackson \& Dennis, 2017; Morland-Schultz \& Hill, 2000). In Western cultures, breasts often are sexualized and objectified (Foss, 2017; Young, 2003). Given this cultural view, some women fear that breastfeeding will make their breasts appear less attractive or that they will be seen by society as more "maternal" and less "sexy" (Stearns, 1999; Young, 2003).

In some cases, anatomical or physiological problems or deformities make breastfeeding more difficult, or may prevent it altogether (WHO, 2009). First, about $10 \%$ of women have "flat" or "inverted" nipples, which may increase the difficulty of breastfeeding due to the baby having trouble latching on to the breast (Alexander, Grant, \& Campbell, 1992; WHO, 2009). Most lactation consultants suggest, however, that with the proper education, support, and (in some cases) tools such as the nipple shield, women with nearly all shapes and sizes of breasts and nipples can breastfeed (Wilson-Clay, 1996). Second, a small number of women develop physiological problems following complications from delivery that inhibit milk production, such as endocrine dysfunction following hemorrhage or retained placenta (WHO, 2009). Third, women with a history of breast cancer may not be able to breastfeed, or may have more difficulty breastfeeding, depending upon the specific treatments they received. For example, women with a history of bilateral total mastectomy are not able to breastfeed, but there have been reports of women breastfeeding following single mastectomy (Shaw, 2011). Contrary to widespread belief, having a history of cosmetic breast augmentation is not associated with lactation problems (Lund, Turkle, Jewell, \& Murphy, 2016). Finally, women with certain infectious diseases are advised against breastfeeding due to the risk of disease transmission to the infant. Specifically, 
breastfeeding is contraindicated among women with human immunodeficiency virus (HIV), human T-cell lymphotropic virus Types I or II, active tuberculosis, varicella, or herpes simplex virus lesions on the breast or nipple (American College of Obstetrics and Gynecology Committee on Obstetric Practice, 2016). Infant-related abnormalities that may inhibit breastfeeding include early jaundice, cleft lip or palate, tongue tie (Hogan, Westcott, \& Griffiths, 2005), muscular weakness, or congenital abnormalities in organ development (e.g., heart, kidney, lung; WHO, 2009). Despite these issues, most women and their babies have the physiological and anatomical ability to breastfeed (WHO, 2009).

The primary alternative to breast milk, infant formula, may help some women circumvent the issues associated with breastfeeding (Brown, Raynor, \& Lee, 2011). Many women report that using formula allows them to delegate childcare tasks, such as feeding, to their partner or other family members (Zimmerman \& Guttman, 2000). A manufactured food, formula fulfills an infant's caloric and nutritional needs, but it lacks the immunological and bioactive properties of breast milk that protect the infant against adverse health outcomes (Riordan \& Wambach, 2010). Gastrointestinal symptoms, such as reflux, are more common among formula-fed infants than breastfed infants because the fat in formula is not digested as completely as the fat in breast milk (Chen, Soto-Ramírez, Zhang, \& Karmaus, 2017; Riordan \& Wambach, 2010). In addition, supplemental formula feedings can hinder maintenance of maternal milk supply (Riordan \& Wambach, 2010).

\section{Measuring Breastfeeding}

Historically, breastfeeding behavior has been poorly operationalized and defined, making it difficult to interpret results across studies (Labbok \& Starling, 2012). In the years 2010-2011 alone, about half of journal articles about breastfeeding described the breastfeeding variable 
(e.g., "exclusive" or "any" breastfeeding), but only about one-quarter of them included detailed information about breastfeeding definitions (e.g., ratio of breast milk to formula feedings; Labbok \& Starling, 2012). Therefore, it is important to comprehensively and precisely measure breastfeeding in the current study.

Several frameworks for defining and measuring breastfeeding have emerged. The current study utilized a modified version of the Interagency Group for Action on Breastfeeding's (IGAB) guidelines (Labbok \& Krasovec, 1990; Labbok \& Starling, 2012). The IGAB committee consisted of breastfeeding and lactation experts, who outlined a detailed schema for categorizing breastfeeding (Labbok \& Krasovec, 1990). The six categories are as follows: exclusive; almostexclusive (breast milk plus water, juice or vitamins); high partial ( $\geq 80 \%$ breast milk); medium partial (20-79\% breast milk); low partial $(<20 \%$ breast milk); and token (infrequent and short durations of breastfeeding; Labbok \& Krasovec, 1990). In recent years (after IGAB categories were established), the American Academy of Pediatrics published guidelines recommending vitamin D supplements to exclusively breastfed babies to prevent rickets and bone fractures (Holick et al., 2011; Wagner \& Greer, 2008). Therefore, the present study used a slightly modified version of the IGAB guidelines, such that babies who received vitamins or medications per healthcare provider recommendation, and nothing else other than breastmilk, were categorized as "exclusive." In addition, the present study further broke down the "mediumpartial" category into more specific groupings, as later described. The IGAB also recommended delineating between the infant feeding at-breast (latched directly on to mother's breast) or consuming milk from a bottle that has been expressed from the breast by hand or breast pump (Labbok \& Krasovec, 1990). There are additional health benefits associated with at-breast feeding for the infant, such as enhanced jaw muscle, oral cavity, and mandible development 
(Buckley \& Charles, 2006; Peres, Cascaes, Nascimento, \& Victora, 2015). For the mother, atbreast feeding is associated with a lowered risk of mastitis/breast trauma, and increased feelings of relaxation after feedings as compared to manual or electric milk expression (Buckley \& Charles, 2006). See Table 1 for the items in the postpartum interview that correspond with the various IGAB breastfeeding categories.

\section{Factors Associated with Breastfeeding}

Breastfeeding outcomes are embedded in a complex network of federal, state, and local policies, as well as cultural and social practices. As such, there are socio-economic, racial, ethnic, and geographic disparities in breastfeeding (CDC, 2016). For example, in the United STates, African American women are less likely to breastfeed than white women (Jones, Power, Queenan, \& Schulkin, 2015; Kogan, Singh, Dee, Belanoff, \& Grummer-Strawn, 2008). Similarly, women living in rural regions (e.g., most of West Virginia) are less likely to initiate and maintain breastfeeding than women living in urban areas (Barton, 2001; Chertok, Luo, Culp, \& Mullett, 2011; Flower, Willoughby, Cadigan, Perrin, Randolph, \& Family Life Project Investigative Team, 2008; Sparks, 2010; Wiener \& Wiener, 2011). Disparities exist internationally as well; almost all women in Scandinavian countries initiate breastfeeding, and nearly half of them continue to breastfeed exclusively for six months. On the other hand, women in developing countries, such as Somalia and Thailand, are less likely to ever exclusively breastfeed (WHO, 2017a).

Increased breastfeeding also is associated with higher maternal education and income, being married (Dubois \& Girard, 2003; Mathews, Leerkes, Lovelady, \& Labban, 2014), and higher parity (Jessri, Farmer, Maximova, Willows, \& Bell, 2013). Parity refers to the number of times a woman has given birth (Bai, Wong, Bauman, \& Mohsin, 2002). More specifically, 
"primiparous" refers to women pregnant with their first child, and "multiparous" describes women who have previously given birth. In addition, women over the age of 25 years are more likely to breastfeed than younger women (Jones, Kogan, Singh, Dee, \& Grummer-Strawn, 2011; Newton, Chaudhuri, Grossman, \& Merewood, 2009). In terms of employment, women with paid maternity leave are more likely to breastfeed, and for longer durations (Baker \& Milligan, 2008; Calnen, 2007; Huang \& Yang, 2015; Scott et al., 2006). Similarly, paid lactation breaks positively affect breastfeeding outcomes (Heymann, Raub, \& Earle, 2013).

Birth-related factors associated with increased breastfeeding include having a vaginal delivery, decreased use of analgesic medication (e.g., epidural) during labor and delivery, and having a full-term infant (Rowe-Murray \& Fisher, 2002). Women who have cesarean section deliveries face distinct challenges associated with breastfeeding. Pain and discomfort associated with surgery may make it difficult for a woman to comfortably position her infant for breastfeeding, or might delay breastfeeding initiation (Heck, Schoendorf, Chávez, \& Braveman, 2003). While cesarean section deliveries and analgesics during labor might make it more difficult to initiate breastfeeding, the effects of these birth-related factors on breastfeeding duration are minimal (Dozier et al., 2013; Sakalidis et al., 2013). In addition, breastfeeding is more likely among women who give birth in a hospital whose policies support breastfeeding via adherence to breast milk supplementation guidelines, employing staff with adequate lactation training, and facilitating skin-to-skin contact between mother and baby in the immediate postpartum period, as well as allowing and encouraging "rooming in," or newborn and mother sleeping in the same hospital room (Abrahams \& Labbok, 2009). Early skin-to-skin contact and "rooming in" are associated with higher rates of initiation and duration of breastfeeding (Chiou, Chen, Yeh, Wu, \& Chien, 2014; Robiquet et al., 2016). 
Preterm infants, or babies born before the $37^{\text {th }}$ week of gestation (Phillips et al., 2013), may have problems with breastfeeding for several reasons. Premature babies' underdeveloped musculature and neurophysiology may make it difficult for them to latch on to the nipple, suck, and swallow (Buckley \& Charles, 2006), and they may have difficulty staying alert during feedings (Wooldridge \& Hall, 2003). Mothers of preterm babies may have to express milk by hand or with a pump for several weeks until the baby is physiologically able to feed at-breast (Wight, 2015). In addition, preterm infants often are hospitalized in the neonatal intensive care unit (NICU). Mothers of babies admitted to the NICU may have difficulty finding time to breastfeed due to the strict schedules and routines of medical check-ups and tests (Flacking, Ewald, Nywvist, \& Starrin, 2005).

The study of the behavioral and psychological determinants of breastfeeding appeals to many researchers because, unlike many other more socially-determined or "fixed" factors (e.g., geographic location, income), behavioral factors are potentially modifiable (Mathews et al., 2013; O’Brien, Buikstra, \& Hegney, 2008). For example, not smoking and maintaining a healthy pre-pregnancy weight are associated with increased breastfeeding (Amir \& Donath, 2012), and there are effective interventions for decreasing smoking among pregnant women (Einarson \& Riordan, 2009), as well as for supporting weight loss among women who are trying to conceive (Lee \& Koren, 2010).

Psychological factors associated with more breastfeeding include holding positive attitudes towards breastfeeding (Guo, Wang, Liao, \& Huang, 2016; Jessri, Farmer, Maximova, Willows, \& Bell, 2013), more knowledge about breastfeeding (Chezem, Friesen, \& Boettcher, 2003; Wallenborn, Ihongbe, Rozario, \& Masho, 2017), and increased breastfeeding perceived behavioral control (Blyth et al., 2002; Ertem et al., 2001; Guo, Wang, Liao, \& Huang, 2016; 
Meedya, Fahy, \& Kable, 2010). Women with more social support also are more likely to breastfeed, and for longer durations, compared to those with less social support (Brown, 2014). Postpartum depression is associated with a decreased likelihood of breastfeeding (Chaput, Nettel-Aguirre, Musto, Adair, \& Tough, 2016; Dennis \& McQueen, 2007; Hatton et al., 2005). Breastfeeding education for patients has been shown to effectively increase breastfeeding initiation via an increase in positive attitudes towards breastfeeding (Ryser, 2004). Usually, breastfeeding education includes instructions on how to breastfeed, and video or in vivo demonstrations of effective breastfeeding techniques or milk expression strategies (Riordan \& Wambach, 2010; Rosen, Krueger, Carney, \& Graham, 2008). Breastfeeding perceived behavioral control, too, can be improved with patient education, which in turn increases breastfeeding duration (Noel-Weiss, Rupp, Cragg, Bassett, \& Woodend, 2006).

\section{Policy Support of Breastfeeding}

Federal and state. In the United States, there are several types of breastfeeding legislation: (a) laws that define breastfeeding as a right, (b) laws that decriminalize breastfeeding in public as a crime of "indecency," (c) workplace lactation policies, and (d) laws that ensure inclusion of lactation services in healthcare plans (American Academy of Pediatrics, 2013; Chertok \& Hoover, 2009; National Conference of State Legislatures, 2017). Currently, 49 states and the District of Columbia have laws that grant women the right to breastfeed in any public or private location in which she and the baby otherwise have a right to be, and 29 states exempt breastfeeding from public indecency laws (National Conference of State Legislatures, 2017).

The Patient Protection and Affordable Care Act (PPACA, 2010) contains several provisions that support breastfeeding women. The PPACA mandates that new health insurance plans included prenatal and postpartum lactation consultation and education as covered services 
at low or no cost to the patient (American Academy of Pediatrics, 2013; Patient Protection and Affordable Care Act, 2010). Similarly, the PPACA requires that new health insurance plans provide a breast pump to all pregnant women at low or no cost (American Academy of Pediatrics, 2013). The PPACA also amended the Fair Labor Standards Act (1938) to require that employers allow lactating women to take breaks to express milk for one year after the birth of the infant, but the breaks need not be paid (PPACA, 2010). The employer also must provide a private space other than the restroom for milk expression (PPACA, 2010).

Although the PPACA was groundbreaking in its explicit support and protection of the rights of lactating women, it has not been without criticism. According to political scientist Courtney Jung (2015), the PPACA's lactation initiatives are more "pro-pumping," than "probreastfeeding," and the law "compel[s] mothers to square the circle between the absence of maternity leave and the injunction to breastfeed exclusively for six months by pumping breast milk at work" (Jung, 2015, p. 137). Jung posits that the real solution for improving women's quality of life and increasing breastfeeding duration is to improve maternity leave policies in the United States. Despite maternity leave being a known correlate of breastfeeding (Baker \& Milligan, 2008; Calnen, 2007; Huang \& Yang, 2015; Scott et al., 2006), the United States is one of the few countries in the United Nations that lacks mandates for paid maternity leave (Huang \& Yang, 2015; US Department of Health and Human Services, 2011).

Over half (53\%) of infants born in the United States participate in the Special Supplemental Nutrition Program for Women, Infants, and Children (WIC; U.S. Department of Agriculture, 2016). Substantial funds have been put towards breastfeeding support programs through WIC, and the rates of breastfeeding have increased dramatically over the past 15-20 years (Jensen, 2012; Ryan \& Zhou, 2006). Still, rates of breastfeeding among WIC participants 
are lower than they are among non-participants (Gregory, Gross, Nguyen, Butz, \& Johnson, 2016; Jensen, 2012). In 2007, the food packages provided to WIC participants were revised to better reflect guidelines for fruit and vegetable intake, as well as to provide breastfeeding women with additional food. The rationale for providing breastfeeding women with more food was that they needed more calories to sustain lactation than their formula-feeding peers, and to create an incentive to breastfeed (Special Supplemental Nutrition Program for Women, Infants and Children, 2014). Preliminary data suggest that the change in food packages has positively affected breastfeeding outcomes (Langellier, Chaparro, Wang, Koleilat, \& Whaley, 2014)

Hospital-based policy. Hospital policies also impact breastfeeding outcomes (Abrahams \& Labbok, 2009; Howe-Heyman \& Lutenbacher, 2016). "Baby-Friendly Hospitals" are certified by UNICEF and the WHO as providing evidence-based perinatal care that facilitates breastfeeding and mother-baby bonding (Nickel, Labbok, Hudgens, \& Daniels, 2013; WHO, 2017b). Becoming a Baby-Friendly Hospital requires extensive staff education and training, and in many cases, major policy changes (Abrahams \& Labbok, 2009; Parry, Tully, Moss, \& Sullivan, 2017). Hospitals that have been certified as "Baby-Friendly" adhere to the "Ten Steps to Successful Breastfeeding," developed by an expert panel (Baby-Friendly USA, Inc., 2016; Hansen et al., 2013; Nickel et al., 2013). These steps, quoted from Nickel et al. (2013, p. 60) include:

Step 1 . Have a written breastfeeding policy that is routinely communicated to all health care staff.

Step 2. Train all health care staff in the skills necessary to implement this policy. Step 3. Inform all pregnant women about the benefits and management of breastfeeding. Step 4. Help mothers initiate breastfeeding within one hour of birth. 
Step 5. Show mothers how to breastfeed and how to maintain lactation, even if they are separated from their infants.

Step 6. Give infants no food or drink other than breast-milk, unless medically indicated.

Step 7. Practice rooming in - allow mothers and infants to remain together 24 hours a day.

Step 8. Encourage breastfeeding on demand.

Step 9. Give no pacifiers or artificial nipples to breastfeeding infants.

Step 10. Foster the establishment of breastfeeding support groups and refer mothers to them on discharge from the hospital or birth center.

In 2016, approximately $18 \%$ of births in the United States occurred in a "baby-friendly" hospital (WHO, 2017b).

\section{Behavioral Breastfeeding Interventions}

The breastfeeding promotion intervention literature is vast, attesting to its importance. As such, the present section will focus on relevant findings from the past 15 years. On the whole, breastfeeding promotion and education interventions are effective in enhancing breastfeeding outcomes (Cartwright, Atz, Newman, Mueller, \& Demirci, 2017; Haroon, Das, Salam, Imdad, \& Bhutta, 2013; Wouk, Tully, \& Labbok, 2017). To date, most interventions designed to enhance breastfeeding outcomes have been delivered in the intrapartum or postpartum period (Hannula, Kaunonen, \& Tarkka, 2008). The formats of these interventions vary considerably, and include individual sessions with lactation consultants (Bass, Rodgers, \& Baker, 2014), home-based visits throughout pregnancy and the postpartum period (da Silva, Nunes, Schwartz, \& Giugliani, 2016; Rojjanasrirat, Nelson, \& Wambach, 2012), and group prenatal classes (Rosen et al., 2008). Due to heterogeneous interventions and study designs, 
however, it is difficult to draw conclusions about which type of breastfeeding intervention is most effective for promoting a specific outcome (i.e., initiation, avoiding in-hospital formula supplementation, exclusivity, duration; Lumbiganon et al., 2012; Wouk et al., 2017).

In a systematic review, Haroon and colleagues (2013) summarized outcomes of randomized-controlled and quasi-experimental trials of breastfeeding promotion interventions. This review included breastfeeding interventions delivered by lay counselors, peer counselors, or healthcare professionals (e.g., lactation consultant, registered nurse) in individual and/or group settings. Results suggested that breastfeeding promotion interventions were most effective in increasing rates of exclusive breastfeeding through the first five months postpartum (Haroon et al., 2013). In general, interventions had more of an effect on exclusive breastfeeding than on partial breastfeeding. Interventions that included individual and group components were more effective than either individual or group sessions on their own, but this could have been due to total amount of contact time with the educator/healthcare provider (Haroon et al., 2013). More recent reviews suggest that the key ingredients for effective breastfeeding promotion interventions are breastfeeding education and interpersonal or social support (Ugurlu \& Yavan, 2016; Wouk et al., 2017).

Peer support groups for breastfeeding are common (Woodman, Bayne, \& MacDonald, 2014). The content and delivery of peer support groups range tremendously in their structure and intensity, but many women find that participating in peer support groups help them to become more confident about breastfeeding and to reach their breastfeeding goals (Dennis, 2002; Forster et al., 2014). In a systematic review on the effects of peer support groups on breastfeeding duration, Kaunonen, Hannula and Tarkka (2012) found that peer support was generally effective in increasing breastfeeding duration, particularly when the support was 
delivered in the postpartum period. Not surprisingly, pregnant women were less likely to seek out breastfeeding support groups than postpartum women (Kaunonen et al., 2012).

Another systematic review suggested that prenatal group education was effective in increasing breastfeeding initiation and duration (de Oliveira, Camacho, \& Tedstone, 2001), however, a more recent review indicated that prenatal group education was only effective if it was interactive (Hannula et al., 2008). "Interactive" interventions involve conversations between learner and teacher (Reeve, Gull, Johnson, Hunter, \& Streather, 2004). Thus, interventions such as Motivational Interviewing (MI; Miller \& Rollnick, 2013) are well-suited to increasing breastfeeding initiation and duration.

Clearly, there are several evidence-based interventions for increasing breastfeeding initiation and duration that can be delivered during the prenatal period, however, prenatal breastfeeding education appears to vary greatly by geographic location, type of facility (e.g., WIC center versus private birth center), type of provider (Balyakina, Fulda, Franks, Cardarelli, \& Hinkle, 2016), and amount of specialized lactation training of the healthcare provider (Kramer et al., 2001; Labarere et al., 2006). The usual care in many settings includes an infant feeding assessment prior to discharge from the intrapartum hospital stay, as well as brief follow-up assessments at well-baby visits (Kramer et al., 2001; Traveras et al., 2004).

\section{Theory of Planned Behavior and Breastfeeding}

The Theory of Planned Behavior (TPB; Ajzen, 1988; 1991) posits that human behavior can be predicted through behavioral intention, which is determined by an individual's attitude towards the behavior, perceived control of the behavior, and the individual's perception of the subjective norms of the behavior (Ajzen, 1991). The TPB has been used to guide the conceptualization and behavioral interventions of many health behaviors in a wide variety of 
populations, from adolescent tobacco use (Guo et al., 2007) to treatment adherence among patients with chronic illnesses (Moral et al., 2015). This theory also has been applied to pregnant and postpartum populations with target behaviors such as tobacco use (De Wilde, Maes, Boudrez, Temmerman, \& Clays, 2017; Gantt, 2001) and physical activity (Hausenblas \& Downs, 2004).

The TBP also has been used as a framework for understanding breastfeeding behavior and for guiding the development of behavioral interventions to improve breastfeeding outcomes in a variety of populations, including rural women (Dodgson, Henley, Duckett, \& Tarrant, 2003; Duckett et al., 1998; Giles et al., 2014; Wilhelm, Rodehorst, Stepans, Hertzog, \& Berens, 2008). According to TPB, it is hypothesized that breastfeeding intention is predicted by a woman's perceived control of breastfeeding, her attitudes towards breastfeeding, and her perceptions of the subjective norms about breastfeeding (e.g., breastfeeding viewed as "normal" or "natural"). In turn, breastfeeding intention would then be hypothesized to predict actual breastfeeding behavior. Many studies have measured the association between intention to breastfeed and actual breastfeeding behavior, and found that the two are positively correlated (Thomas-Jackson et al., 2016). Though there has been some disagreement in the field as to whether the attitude, subjective norms, and perceived behavioral control predict breastfeeding intention (Avery, Duckett, Dodgson, Savik, \& Henly, 1998; Göksen, 2002; Kloeblen-Tarver, Thompson, \& Miner, 2002), the only existing (and quite recent) meta-analysis and structural equation modeling performed on this topic suggested a good fit of the entire TPB model as applied to breastfeeding (Guo, Wang, Liao, \& Huang, 2016). Specifically, attitude, subjective norms, and perceived behavioral control were all strong predictors of breastfeeding intention, and intention significantly predicted actual breastfeeding behavior (Guo et al., 2016). 
The TPB was used as a theoretical framework for the present study, and is reflected in the variables targeted in the MI intervention and measured (e.g., attitudes towards breastfeeding, intention to breastfeed, perceived behavioral control of breastfeeding behavior). Other studies have used TPB to guide MI interventions targeting breastfeeding (Wilhelm, Aguirre, Koehler, \& Rodehorst, 2015; Wilhelm, Flanders Stepans, Hertzog, Rodehorst, \& Gardner, 2006).

\section{Motivational Interviewing}

Motivational Interviewing is a psychosocial intervention designed to help individuals increase readiness for behavior change by increasing intrinsic motivation and resolving ambivalence (Hettema, Steele, \& Miller, 2005; Miller \& Rollnick, 2013). MI founders Miller and Rollnick (2013) defined MI as a "collaborative conversation style for strengthening a person's own motivation and commitment to change" (p. 12). MI conversations help clients change behavior through identifying and resolving discrepancies between goal behaviors and actual behaviors. Behavior change is promoted through the elicitation of "change talk," or client-verbalized arguments for change. In MI, change talk is elicited through two major components: the therapeutic relationship, or the embodiment of the "spirit" of MI, and technical skill (Miller \& Rose, 2009).

The "spirit" of Motivational Interviewing. The underlying "spirit" of MI consists of partnership, acceptance, compassion, and evocation (Miller \& Rollnick, 2013). In MI, the therapist and client are seen as partners; the two must work together to achieve a common goal (i.e., client behavior change). This partnership mentality lies opposite to traditional healthcare settings, where the provider typically gives advice about what the patient "should" or "should not" do. According to Miller and Rollnick (2013), MI "is an active collaboration between experts. People are the undisputed experts on themselves. No one has been with them longer, or 
knows them better than they do themselves" (p. 15). MI, therefore, views the client as the expert of her own experience, and tapping into this expertise helps to promote change (Hibbard, Mahoney, Stock, \& Tusler, 2007). Second, compassion means that the helper cares about the client's needs. Third, the spirit of MI is accepting; the helper wholeheartedly accepts the client as a human, regardless of the situation or problems that she has. The final component of the MI spirit is evocation, which is illustrated in MI through the therapist using a strengths-based (as opposed to deficits-based) approach to eliciting client change talk and motivation (Miller \& Rollnick, 2013).

MI's technical aspects. The technical component of MI consists of four processes and five core interviewing skills (Miller \& Rollnick, 2013). The four processes include engaging, focusing, evoking, and planning (Miller \& Rollnick, 2013). The process of engaging occurs when the client and helper establish a working relationship; engagement must be present in order for change to occur (Miller \& Rollnick, 2013). Next, the process of focusing involves developing the direction of the conversation; often, through focusing, the client's specific goal(s) begin to emerge. The next process, evoking, is known as the "heart of MI" (Miller \& Rollnick, 2013, p. 28). Evoking occurs when the client's ideas about a particular behavior change are explored, and the client voices arguments for making the change. In the process of planning, the conversation involves developing an action plan for maintaining behavior change. In this process, the client's autonomy should be emphasized, and the therapist's role is to elicit solutions to anticipated problems from the client (Miller \& Rollnick, 2013). Although the four processes are described sequentially, and in some ways must occur in sequence (e.g., evoking can only occur after the client and therapist have focused their conversation), the four processes are 
"recursive; one does not end as the next begins. They may flow into each other, overlap, and recur" (Miller \& Rollnick, 2013, p. 26).

The five "core skills" of MI are comprised of asking open-ended questions, affirming, reflecting, summarizing, and informing and advising (Miller \& Rollnick, 2013). The therapist's use of these skills is crucial to eliciting change talk (Miller \& Rollnick, 2013). Typically, MI conversations consist of a volley between open-ended questions and reflections. In fact, the recommended ratio of reflections to open-ended questions is 2:1 (Moyers, Manuel, \& Ernst, 2014). Affirmations "recognize and acknowledge that which is good including the individual's inherent worth as a fellow human being" (p. 64). During MI, affirmations may decrease the likelihood of treatment attrition, reduce client defensiveness, and help to facilitate change (Miller \& Rollnick, 2013). MI therapists utilize the skill of summarizing, which involves reflecting several of the client's ideas or sentiments in one phrase or statement. The final core skill of MI is informing and advising. The therapist must use this skill carefully, and be careful to maintain the MI spirit at all times. Miller and Rollnick (2013) recommend that before providing information, the therapist first asks permission, explores what the client already knows and thinks about the particular topic, and asks the client about what, specifically, she is interested in learning more. In regard to breastfeeding, women often report that in addition to wanting support, encouragement, and understanding from their healthcare providers and family members, they also want specific advice about the benefits and practical aspects of breastfeeding (Graffy \& Taylor, 2005). As such, the skill of informing and advising is particularly relevant to the present intervention.

MI and health behavior change. MI has its roots in substance abuse treatment, but also has been effectively used to engender a variety of health behavior changes (Martins \& McNeil, 
2009; McNeil, Addicks, \& Randall, in press). Health behavior can be defined as, "an action taken by a person to maintain, attain, or regain good health and to prevent illness" (Health Behavior, 2009). MI has successfully been used to improve diabetes treatment adherence (Mulimba \& Byron-Daniel, 2014; Steinberg, 2011), increase physical activity (Thompson et al., 2011), increase fruit and vegetable intake (Resnicow et al., 2001), improve oral hygiene (Cascaes, Bielemann, Clark, \& Barros, 2013), and to promote smoking cessation (Hettema \& Hendricks, 2010).

While breastfeeding can be conceptualized as a health behavior, as it does indeed help to "maintain, attain, [and] regain good health and...prevent illness," (Health Behavior, 2009), it can also be conceptualized as a parenting behavior, or a sort of health behavior "by proxy." As such, MI also has been used to change parent behavior to improve child health. Pediatric MI interventions with parent involvement were successful in improving a variety of health behaviors, as compared to comparison groups (Borrelli, Tooley, \& Scott-Sheldon, 2015; Erickson, Gerstle, \& Feldstein, 2005). More specifically, MI with parents/caregivers has been used to enhance oral hygiene practices among parents of preschoolers, which in turn improved the children's oral health (Ismail, Ondersma, Willem, Little, \& Lepkowski, 2011; Naidu, Nunn, \& Irwin, 2015). In addition, MI has been used to help parents make a variety of changes to increase child physical activity, reduce child body mass index (BMI), and reduce "screen time" (Borrelli et al., 2015; Davoli et al., 2013).

In addition to general parent-involved pediatric MI interventions, MI also has been delivered successfully during pregnancy to change other health behaviors. Specific examples include reducing smoking (Hayes et al., 2013; Karatay, Kublay, \& Emiroğlu, 2010) and alcohol consumption (Handmaker, Miller, \& Manicke, 1999), attending preparatory childbirth classes 
(Rasouli et al., 2017), and choosing to deliver at a healthcare facility by a skilled birth attendant rather than at home with an unskilled (e.g., family member) birth attendant in Western Kenya (Gisore, Kaseje, Were, \& Ayuku, 2014).

MI and breastfeeding promotion. MI is relatively new to lactation and breastfeeding promotion, but there is some evidence to suggest that it may be beneficial in enhancing breastfeeding outcomes. There are seven published studies that have explored the feasibility of implementing MI to enhance breastfeeding outcomes, as well as the effectiveness of such interventions. The feasibility (i.e., acceptability and attrition) of these interventions seems to vary substantially by study population and type of therapist. For example, Copeland and colleagues (2016) designed a unique peer-delivered MI intervention, "Mam-Kind," and tested the feasibility and acceptability of the intervention on a sample of women in the UK. Preliminary results (meeting abstract only) suggested that women found the intervention to be helpful, and healthcare providers judged the intervention to be easily integrated into existing systems. Peer supporters, however, described difficulty in implementing MI in a peer-to-peer context.

In addition to acceptability, attrition is another variable frequently used to assess feasibility. Wilhelm et al. (2015) sought to determine whether a three-session, in-home, postpartum MI intervention increased breastfeeding self-efficacy, intent to breastfeed for six months, and actual duration of breastfeeding among rural Mexican-American women. Women assigned to the MI group $(n=26)$ were compared to women assigned to the control group $(n=$ 27), who received information on infant safety and injury prevention. No significant differences between groups existed, however, high rates of attrition in both the MI (69\%) and control (63\%) groups made it difficult to interpret results. The authors suggested possible reasons for attrition 
were cultural inappropriateness of the intervention, participant discomfort with home visits, and researchers' lack of established relationships with the community in which the study took place.

In terms of effectiveness, most studies suggest that MI may be a promising tool to effect change in breastfeeding behavior, though there are several notable limitations to the existing body of literature. Wilhelm and colleagues (2006) conducted a multi-site randomized controlled trial to determine whether MI could help to promote breastfeeding in a group of primiparous, postpartum women $(N=73)$ in a rural U.S. area. Participants were recruited during the intrapartum hospital stay. Approximately half of the participants were assigned to treatment as usual, and about half were assigned to the MI intervention, which was delivered by a research nurse approximately 2-4 days postpartum. This intervention also included brief booster sessions at two and six weeks postpartum. The primary outcome measure was total number of days breastfed. At each visit, breastfeeding in the past 24 hours was confirmed using infant test weights. Results indicated that participants in the MI group breastfed for more days than the comparison group, but this difference was not significant. One major strength of this study was that the researchers validated participant self-report of breastfeeding with the test weight procedure (Chapman \& Pérez-Escamilla, 2000). Other strengths of the study were its multiple measurement time points and comprehensive measure of baseline variables that could affect breastfeeding (e.g., socio-economic status, employment status). In terms of limitations, this study did not include measures of treatment integrity, and there were some differences between research sites that may have impacted results (e.g., one site conducted the intervention in-home and the other did so at the hospital).

In another randomized-controlled trial, researchers in Turkey sought to determine the effectiveness of a "Breastfeeding Motivation Program" on increasing breastfeeding initiation and 
duration among primiparous women (Cangöl \& Şahin, 2017). Half $(n=34)$ of the participants were randomly assigned to the "Breastfeeding Motivation Program," which consisted of three inperson sessions (third trimester of pregnancy, one day postpartum, one month postpartum) and one follow-up phone call (four months postpartum). Participants in the control group $(n=33)$ learned about self-breast examinations. Results suggested that compared to the control group, women assigned to the "Breastfeeding Motivation Group" were more likely to endorse intending to breastfeed, experience more anxiety about breastfeeding, initiate breastfeeding earlier (sooner after delivery), experience fewer breastfeeding problems, and report higher breastfeeding selfefficacy than those in the control group. The authors did not include effect sizes in their results, however, their original power analysis was based on a medium effect size (Cohen's $d=.3$ ). Women in the "Breastfeeding Motivation Group" were slightly more likely to be breastfeeding at four months postpartum than those in the control group, but this difference was not statistically significant. Strengths of this study include measuring perceived importance to breastfeed (although not statistically significant between groups), intention to breastfeed and actual breastfeeding behavior, and having an intervention that spanned the prenatal, intrapartum, and postnatal periods. While this study offered promising results, it had several major flaws. First, it is unclear whether the therapists in the current study had any formal MI training, as none is described. Second, the authors describe the "Breastfeeding Motivation Program" as being MI, however, according to the author's diagram outlining the intervention, the program was more structured and less patient-centered than the creators of MI intended (Miller \& Rollnick, 2009). Third, the study contained no measures of treatment integrity. Finally, the only sociodemographic characteristics measured were education and "economic level," and these sociodemographic characteristics were not included as covariates for any of the analyses. 
Targeting a typically underserved population, Tuthill and colleagues (2017) conducted a prenatal (third trimester) randomized-controlled trial of MI to promote exclusive breastfeeding among women with HIV in South Africa. The intervention group $(n=29)$ received standard prenatal education on prevention of mother-to-child transmission of HIV, as well as one session of MI focused on resolving ambivalence about and increasing motivation for exclusively breastfeeding. The control group $(n=29)$ received the standard education only. Primary outcome measures included breastfeeding knowledge and skills, breastfeeding motivation, breastfeeding self-efficacy, and actual breastfeeding behavior at six weeks postpartum. Breastfeeding intention, knowledge, motivation, and behavior were higher in the MI group, though the results were not statistically significant. Interestingly, and in accordance with the TPB, breastfeeding intention and self-efficacy were correlated with exclusive breastfeeding behavior, regardless of group assignment and after controlling for breastfeeding attitudes, adjusted $O R=1.425,95 \% \mathrm{CI}=[1.007,2.015], p=.045$. Strengths of this study include targeting a high-risk, underserved population, cultural adaptation of the intervention and assessment instruments, measuring both intention and actual breastfeeding behavior, describing the formal MI training received by the therapists, and conducting the intervention during pregnancy. On the other hand, the study included no measure of MI treatment integrity, and few details about the intervention, including length, were provided.

On a larger scale, Elliott-Rudder, Pilotto, McIntyre, and Ramanathan (2014) conducted a cluster randomized-controlled trial of MI during regular well-child visits when the baby was two, four, and six months old. A total of 15 family practices $(N=330$ individual participants $)$ in Australia were randomized into either the MI group (7 practices) or the treatment as usual control group (8 practices). Results indicated that women assigned to the MI group were more 
likely to self-report exclusive breastfeeding at four months postpartum, $O R=1.88,95 \% \mathrm{CI}=$ [1.01, 3.50], $p=.047$ (equivalent to small Cohen's $d$; Chen, Cohen, \& Chen, 2010). There were no differences between groups for any breastfeeding or exclusive breastfeeding at the six-month postpartum measurement point. Strengths of this study include large sample size, minimal attrition, seemingly convenient integration into existing well-child visit structure, and welldescribed MI training and supervision of therapists. The limitations of this study included poorly outlined MI procedures, lack of MI treatment integrity measures, and lack of an attention-control group.

Taken together, the results of these studies suggest that there is some evidence for the feasibility and effectiveness of using MI to promote breastfeeding in a variety of populations, but that this conclusion is far from certain. None of the studies included MI treatment integrity measures, and only two studies (Cangöl \& Şahin, 2017; Wilhelm et al., 2006) included a sample protocol of the actual MI session. More research with clear MI-adherent treatment protocols is needed to fully elucidate MI's effectiveness for promoting breastfeeding.

\section{Rationale for Motivational Interviewing During Pregnancy}

Although receiving information and advice from healthcare professionals is effective in increasing breastfeeding initiation and duration (Hannula et al., 2008), many new mothers report that the advice they receive from healthcare providers is inconsistent (George, 2005; Hauck, Graham-Smith, McInerney, \& Kay, 2011; Nelson, 2007; Schmied, Beake, Sheehan, McCourt, \& Dykes, 2011). Results of many studies suggest that providing information is "not enough" and in fact can be confusing, frustrating, and discouraging for new mothers, and can negatively affect breastfeeding outcomes (Bramhagen, Axelsson, \& Hallström, 2006; Cronin, 2003; Graffy \& Taylor, 2005). MI challenges the tendency of healthcare providers to give advice, and rather acts 
as a guide by supporting, motivating, encouraging, and eliciting change talk from the client (Miller \& Rollnick, 2013). In addition, MI allows for information-giving and advising with the permission of the client (Miller \& Rollnick, 2013). Given that breastfeeding promotion interventions are most effective if they are interactive (Hannula et al., 2008), MI's conversationbased style shows promise for improving outcomes.

The "breast is best" message touted by breastfeeding advocates and ingrained in recent health policies conveys the medical and immunological benefits associated with breastfeeding, but also "carries moralistic dimensions" (Thomson, Ebisch-Burton, \& Flacking, 2015, p. 33). Many parents learn to associate breastfeeding with being a "good mother," and to associate lack of breastfeeding with failure, or being a "bad mother" (Mozingo, Davis, Droppleman, \& Merideth, 2000). Language used in the scientific literature often reflects these moralistic sentiments. Many articles in this arena describe breastfeeding as a "success" and/or lack of breastfeeding as a "failure." Exposure to these moralistic, binary semantics may in turn cause women to feel ashamed, guilty, anxious, or embarrassed for not reaching their own breastfeeding goals or the ones that they perceive society has set for them (Thomson et al., 2015). Referencing government-funded breastfeeding promotion programs, political scientist Jung (2015) wrote, "not one of the public breastfeeding initiatives since 1995 seems directed toward supporting women's own preferences and choices" (pp. 124). The non-judgmental and person-centered style of MI may help to counteract these feelings of shame, embarrassment, and lack of autonomy (Miller \& Rollnick, 2013).

Practically speaking, MI is an ideal choice for targeting perinatal women because it can be delivered effectively in primary care, obstetric, and in-home settings (Emmons \& Rollnick, 2001). Delivery of brief interventions in routine settings, such as primary care, is crucial when 
targeting lactation outcomes, as many women do not need, want, or have access to outside lactation resources (Friesen, Hormuth, Petersen, \& Babbitt, 2015). Recently, the U.S. Preventive Services Task Force (2016) published formal recommendations that encourage primary care clinicians to support breastfeeding in their patients:

Primary care clinicians can support women before and after childbirth by providing interventions directly or through referral to help them make an informed choice about how to feed their infants and to be successful in their choice. Interventions include promoting the benefits of breastfeeding, providing practical advice and direct support on how to breastfeed, and providing psychological support. Interventions can be categorized as professional support, peer support, and formal education, although none of these categories are mutually exclusive, and interventions may be combined within and between categories. (pp. 1689-1690)

The American College of Obstetrics and Gynecology (ACOG) released a similar statement emphasizing the importance of lactation care being included in regular obstetric practice, including support, education, and encouragement of breastfeeding before and after childbirth (ACOG Committee on Obstetric Practice, 2016). Notably, the ACOG specifically recommends engaging in "patient-centered" discussions about breastfeeding. Without using "MI" language, the ACOG Committee on Obstetric Practice encouraged providers to engage patients using MI-consistent conversations: "Beginning conversations about lactation early in prenatal care by asking the patient and her family, 'What have you heard about breastfeeding?' sets the stage for a patient-centered discussion" (pp. 3). It would seem, then, that healthcare providers in several specialties have goals in line with MI to support and enhance breastfeeding. 


\section{Rationale for a Single Session Intervention}

Research has shown that a single session of MI can be effective for modifying behavior, such as reducing alcohol (Schilling, El-Bassel, Finch, Roman, \& Hanson, 2002) and drug use (McCambridge \& Strang, 2004), smoking cessation (Lai, Cahill, Qin, \& Tang, 2010; LindsonHawley, Thompson, \& Begh, 2015), enhancing engagement in substance abuse treatment (Carroll, Libby, Sheehan, \& Hyland, 2001), increasing self-efficacy for substance abstinence in a drug detoxification unit (Berman, Forsber, Durbeej, Källmén, \& Hermansson, 2010), and decreasing ambivalence towards safe sex practices among individuals with HIV (Rutledge, 2007). In fact, two Cochrane reviews (Lai et al., 2010; Lindson-Hawley et al., 2015) found that in the case of smoking cessation, multiple sessions of MI may be marginally more effective, but that single sessions still yield positive outcomes and require fewer resources. Even more relevant to the present study, there have been several single-session MI studies implemented during pregnancy to decrease prenatal alcohol use. Single-session MI interventions to reduce alcohol use during pregnancy were found to be feasible, but due to low baseline alcohol consumption, no significant effects of MI were found (Handmaker et al., 1999; Osterman, Carle, Ammerman, \& Gates, 2014; Osterman \& Dyehouse, 2012).

Practically speaking, a single session of MI is more conducive to implementation in primary care/obstetric settings than a multiple-session intervention. Although breastfeeding may be a priority for many clinicians, they may not have time to spend multiple visits providing breastfeeding education and support. As such, developing an effective one-session intervention may help to increase participation from primary care, obstetric, and pediatric healthcare providers. 


\section{Rationale for a Rural, Appalachian Target Population}

The current study's target population of primarily rural, Appalachian women is important for several reasons. First, none of the other studies assessing MI effectiveness focused on Appalachian samples, making it an area where more knowledge is needed. Given that breastfeeding is a culturally-bound practice (Gail \& Krell, 2014; Kim, Fiese, \& Donovan, 2017), and Appalachia is a geographic region with unique cultural practices, MI may have different outcomes there than in other U.S. populations (McNeil, Crout, \& Marazita, 2012).

Second, adverse perinatal and early childhood health outcomes disproportionately affect Appalachian families (McNeil et al., 2012). These adverse health outcomes include increased risk of infant mortality (Yao, Matthews, \& Hillemeier, 2012), higher prevalence of childhood obesity (Berlin, Hamel-Lambert, \& DeLamatre, 2013; Montgomery-Reagan, Bianco, Heh, Rettos, \& Huston, 2009), and increased dental caries during pregnancy and early childhood (Neiswanger et al., 2015; Polk, Kim, Manz, \& Weyant, 2015), among others. Breastfeeding decreases the risk for many of these negative health conditions (e.g., Ip et al., 2009; Khan, Vesel, Bahl, \& Martines, 2015; Reynolds et al., 2014), and thus interventions to increase breastfeeding may have long-lasting, cascading benefits.

Third, compared to other states, West Virginia lacks state and hospital policy support of breastfeeding (CDC, 2014). West Virginia has only one "Baby-Friendly" hospital (BabyFriendly USA, Inc., 2017). Less than one-quarter of childbirth facilities in West Virginia follow guidelines against provision of non-breast milk nutrition during the intrapartum hospital stay, and less than half of West Virginia facilities annually assess maternity staff for lactation competencies (CDC, 2015). Finally, women living in Appalachia tend to be lower income and have lower levels of education than women in other parts of the country (Appalachian Regional 
Commission, 2017). To illustrate, households in Appalachia in 2015 earned incomes approximately $\$ 15,000$ less than the national average, and are more likely to live below the poverty line (Appalachian Regional Commission, 2017). Given that poverty is a strong correlate of not breastfeeding (Scott et al., 2006) and early cessation of breastfeeding (Newhook et al., 2017), low-income women are an important public health target. Compounding the low income of many women in Appalachia is poor access to prenatal care (Snyder \& Thatcher, 2014).

\section{Statement of the Problem}

Breastfeeding initiation and duration rates fall short of WHO and AAP recommendations, particularly in the Appalachian region (Chertok et al., 2011; Wiener \& Wiener, 2011). Several studies suggest that MI is an effective, non-judgmental approach to supporting behavior change in perinatal women (Handmaker et al., 1999; Karatay et al., 20101). Many women decide on how to feed their babies during pregnancy (Chantry, Eglash, \& Labbok, 2015), and breastfeeding intention is greatly influenced by the education, support, and encouragement of prenatal healthcare providers (Taveras et al., 2003). Still, existing knowledge on the effectiveness of prenatal MI for breastfeeding promotion is unclear.

\section{Aims of the Proposed Study}

The central aims of this study were to determine the feasibility and preliminary effectiveness of a single session lactation-focused MI delivered during the third trimester of pregnancy in enhancing breastfeeding initiation, duration, and exclusivity at one month postpartum. Targets of the MI intervention were based on the TPB, such as increasing perceived behavioral control, enhancing attitudes towards and perceived subjective norms about breastfeeding, and increasing intention to breastfeed. The MI group was compared to the psychoeducation group, which consisted of minimally-interactive, general education about infant 
development and developmental stages (National Center for Infants, Toddlers and Families, 2014). The specific research questions, guided by the TBP framework, and corresponding hypotheses, are presented in the temporal order in which the dependent variables were measured: infant feeding intention, breastfeeding perceived behavioral control and importance, infant feeding knowledge, knowledge of infant development, breastfeeding attitudes, breastfeeding initiation, and infant feeding status at one month postpartum.

Feasibility. The first objective of the present study was to evaluate the overall feasibility of conducting a one-session prenatal MI session. Given the open-ended nature of this question, there were no hypotheses. In accordance with current guidelines on the potential areas of assessment in feasibility studies, areas of evaluation included intervention demand, positive and negative effects on participants, practicality, factors affecting implementation, attrition, and total resources needed to complete the project (Arain, Campbell, Cooper, \& Lancaster, 2010; Bowen et al., 2009; Shanyinde, Pickering, \& Weatherall, 2011).

Research question 1: Intention. Compared to the psychoeducation group, how does MI affect infant feeding intentions regarding both method (e.g., formula, breast milk, mix) and intended breastfeeding duration?

Hypothesis 1. Compared to women assigned to the psychoeducation group, women in the MI group will be more likely to change their feeding plans, such that they will change from intending to formula feed to breastfeeding, either partially or exclusively. In addition, for women who plan to breastfeed or who have not yet decided on how they plan to feed their babies, those in the MI group will achieve greater gains in intended breastfeeding duration (in months). 
Research question 2: Perceived behavioral control. Compared to the psychoeducation group, does MI affect breastfeeding perceived behavioral control?

Hypothesis 2. Women who receive MI will achieve greater gains in breastfeeding perceived behavioral control than those who receive psychoeducation.

Research question 3: Importance. Compared to the psychoeducation group, does MI affect perceived breastfeeding importance?

Hypothesis 3. Women who receive MI will achieve greater gains in breastfeeding perceived breastfeeding importance than women who received psychoeducation.

Research question 4: Infant feeding knowledge. Compared to the psychoeducation group, does MI differentially affect infant feeding knowledge?

Hypothesis 4. Women who receive the MI intervention will achieve greater gains in infant feeding knowledge than those in psychoeducation group.

Research question 5: Attitudes. Compared to a psychoeducation intervention, does MI differentially affect breastfeeding attitudes and perceived subjective norms?

Hypothesis 5. Women who receive the MI intervention will achieve greater gains in breastfeeding attitudes and perceived subjective norms than those in the psychoeducation group.

Research question 6: Knowledge of infant development. Compared to the psychoeducation session, does MI differentially affect knowledge of infant development?

Hypothesis 6. Women in the psychoeducation group will achieve greater gains in knowledge of infant development than those in the MI group.

Research question 7: Initiation. Compared to women assigned to the psychoeducation group, how does MI affect breastfeeding initiation? 
Hypothesis 7. Compared to women assigned to the psychoeducation group, women assigned to the MI group will be more likely to initiate breastfeeding.

Research question 8: Infant feeding at one month postpartum. Compared to women assigned to the psychoeducation group, how does MI affect infant feeding status at one month postpartum?

Hypothesis 8. Compared to women assigned to the psychoeducation group, women in the MI group will be more likely to be classified as "exclusive"/“almost-exclusive" or "partial" breastfeeders, (Labbok \& Krasovec, 1990) than those in the psychoeducation group.

\section{Method}

\section{Design}

The project was a 2 (Group: MI, psychoeducation) x 3 (Time of measurement: preintervention, post-intervention, one month postpartum) single-blind randomized controlled trial. At the in-person meeting (third trimester of pregnancy), data were collected for the following six outcomes, both pre- and post- intervention: (a) infant feeding intentions, (b) breastfeeding perceived behavioral control, (c) perceived breastfeeding importance, (d) infant feeding knowledge, (d) breastfeeding attitudes and subjective norms, and (f) knowledge of infant development.

At the time of the postpartum phone call (one month postpartum), data were collected for the two final dependent variables: breastfeeding initiation (yes/no), and category of infant feeding at one month postpartum. To accurately categorize the type of infant feeding, the participant was asked to describe a variety of details, such as the proportion of feedings that were breast milk; duration of breastfeeding; use of expressed breast milk; frequency of feedings; inhospital formula supplementation; infant consumption of water, vitamins, and minerals; and 
timing of introduction of other foods and liquids (Labbok \& Krasovec, 1990; Labbok \& Starling, 2012).

Covariates. Many potential covariates also were collected. These covariates were determined by examining major predictors and correlates of breastfeeding (see Jessri et al., 2013 and Mathews et al., 2014 for comprehensive reviews). See Table 7 for baseline demographic characteristics, Table 8 for breastfeeding-related experiences and perceptions, Table 9 for health and mental health variables, and Table 10 for baby- and birth-related variables measured at one month postpartum.

\section{Sample}

The MI and breastfeeding promotion literatures were examined to determine an acceptable sample size for the present study. Previous studies testing the utility of MI for breastfeeding had total sample sizes ranging from 53 (Wilhelm et al., 2015) to 330 (ElliottRudder et al., 2014), with most studies' sample sizes not exceeding 75 participants.

Effect sizes in these studies were in the small to medium range $(d=.24-.30, O R=1.40$ 1.81). More generally speaking, effect sizes in MI randomized-controlled trial literature vary greatly $(d=.10-.90 ; O R=1.10-3.20)$, depending upon the target behavior to change, the type of provider who delivers the intervention (i.e., psychologist versus nurse versus trained undergraduate), and the strength of the control group intervention (Burke, Arkowitz, \& Menchola, 2003; Lundahl \& Burke, 2009; Rubak, Sandbaek, Lauritzen, \& Christensen, 2005). Effect sizes among studies examining effects of lactation training or breastfeeding promotion interventions on breastfeeding outcomes varied similarly (Bonuck, Trombley, Freeman, \& McKee, 2005; Bonuck et al., 2014; Chung, Raman, Trikalinos, Lau, \& Ip, 2008). Effect sizes of MI tend to decrease as the temporal distance increases between the intervention and 
measurement time point. Considering previous literature, as well as the fact that the dependent variables were measured only 2-4 months following the intervention, the desired effect size for the present study was set at .65. According to Cohen's (1988) guidelines, an effect size of $d=$ .65 is considered medium to large.

The power analysis program GPower 3 (Faul, Erdfelder, Lang, \& Buchner, 2007) was used to determine the necessary sample size for the present study. An a priori GPower calculation revealed that to detect differences between groups with power of .8 , effect size of $d=$ .65 , and $\alpha=.05$, the needed sample size was approximately 78 participants $(n=39$ in MI group; $n=39$ in psychoeducation group). As such, approximately 80 participants were recruited, which is a similar sample size to other MI and lactation studies (e.g., Cangöl \& Şahin, 2017, Wilhelm et al, 2006). An a priori decision was made to utilize intention-to-treat analysis in the case of participant dropout during the in-person session or major deviations from the assigned protocol.

Participants were pregnant women at least 18 years of age who were at least 27 weeks pregnant, but had at least one week until their due date (i.e., entered the third trimester of pregnancy; Mayo Foundation for Medical Education and Research, 2014). All participants had singleton pregnancies, as women with multiple births (i.e., twins or triplets) sometimes have more difficulty breastfeeding, and often require more education and support (Fisher \& Stocky, 2003). There was no upper age limit for participation. All participants were able to read, write, and understand English. In addition, all participants were required to have access to a telephone to complete the postpartum phone call.

Participants with certain medical or physical conditions that might have prevented them from being able to understand study procedures and/or to breastfeed were excluded from the study. Examples of such conditions were developmental or intellectual disability, infectious 
disease, and history of mastectomy/lumpectomy (Levison, Weber, \& Cohan, 2014). Women who had been told by their health care professional that their developing fetus had a physical abnormality that could hinder breastfeeding also were excluded. In addition, women taking medications contraindicated for breastfeeding also were excluded (e.g., antiretrovirals, lithium; American Academy of Pediatrics, 2001).

As a part of the study screening process, all potential participants were asked about current use of illicit, non-prescribed substances. Women who endorsed current use of illicit substances were not included in the study due to substance-related contraindications with breastfeeding (Moretti, Lee, \& Ito, 2000). In all these cases $(n=6)$, the women were actively involved in an intensive outpatient addictions treatment program (i.e., WVU Chestnut Ridge Center's Comprehensive Opioid Addiction Treatment program). If ever there had been a case in which a prospective participant endorsed illicit substance use and was not already involved in addictions treatment, efforts would have been made to refer the woman to appropriate addictions treatment to address the health and mental health needs of the woman and her developing fetus, as recommended by the ACOG Committee on Underserved Women (2011). ${ }^{1}$ See Appendix A for a detailed list of exclusion and inclusion criteria.

The research protocol complied with all relevant American Psychological Association guidelines for the ethical treatment of human participants, including the informed consent process. The research protocol was approved by the Institutional Review Board at West Virginia

\footnotetext{
${ }^{1}$ From an ethical perspective, some states have mandating reporting laws that extend to prenatal substance use/abuse, however, in the states in which the present study was conducted (Ohio, Pennsylvania, and West Virginia), prenatal substance abuse lies outside the purview of mandated reporting to child welfare authorities (Child Welfare Information Gateway, 2015).
} 
University (Protocol ID \#: 1605123038). See Appendix B. The trial was registered at clinicaltrials.gov, trial number NCT03033459.

\section{Measures}

Prenatal interview. The semi-structured interview was developed for the purpose of this study. Most of the items in this interview were derived from the CDC's Infant Feeding Practices Study II (Fein et al., 2008). First and foremost, this interview included the pre-intervention measures of infant feeding intentions, perceived behavioral control, and perceived importance. In addition, this interview allowed recording of participant demographic information (e.g., education, income, marital status), employment history and plans, obstetric history (e.g., previous pregnancies, previous live births, previous miscarriages), medical history (e.g., current and past medical groups, current medications), psychosocial history (e.g., history of anxiety, depression, abuse, trauma), past breastfeeding experience, perceived family and healthcare provider support of breastfeeding, employment status, and whether the current pregnancy was planned. This interview took approximately 10-30 minutes to complete, depending upon the participant's response pattern, comprehension level, and style. See Appendix C.

Iowa Infant Feeding Attitudes Scale (IIFAS; Mora, Russell, Dungy, Losch, \& Dusdieker, 1999). The IIFAS is a 17 item self-report measure that assesses general attitudes towards infant feeding methods, including breastfeeding, formula feeding, and combinations of the two. The scale measures several dimensions of infant feeding, including cost, nutrition, convenience, and mother-infant bonding. Permission was granted from the authors to use this scale in the present study. Participants answer items on a five-point Likert scale ranging from "strongly disagree" to "strongly agree." Total scores range from 17 to 84, with higher scores indicating more favorable attitudes towards breastfeeding. Total IIFAS scores were used in the 
present study's analyses. The scale demonstrates good psychometric properties in women, regardless of selected feeding method. Cronbach's alpha was .85-.86 on the original development scale (Mora et al., 1999). Other studies utilizing the IIFAS among pregnant women have demonstrated considerable variation in internal consistency values, with Cronbach's alpha ranging from .50 (Wallis et al., 2008) to .79 (Scott, Shaker, \& Reid, 2004; Sittlington, StewartKnox, Wright, Bradbury, \& Scott, 2007).

Being the first measure of its kind, the authors assessed the IIFAS content validity by creating what they termed a "multi-attribute utility assessment" of infant feeding methods, which entailed rating attributes of breastmilk and formula on several dimensions (e.g., cost, nutritional properties, convenience, impact on sexuality), and rating how important each of these attributes was to the participant personally. Scores on the multi-attribute utility assessment were tabulated in such a way that higher scores indicated a preference for breastfeeding, and lower scores indicated a preference for using formula. Correlational analyses revealed that total scores on the IIFAS were highly correlated $(r=.80)$ with composite scores on the multiattribute utility assessment, which indicated good content validity.

The IIFAS has demonstrated good predictive validity in European American and European samples, with higher scores being associated with an increase in the likelihood of intending to breastfeed, actual breastfeeding behavior during the intrapartum hospital stay (Dungy, McInnes, Tappin, Wallis, \& Oprescu, 2008; Mora et al., 1999), and intent to continue breastfeeding at six weeks and six months postpartum (Wallis et al., 2008). In other populations, such as Latina women, the IIFAS similarly demonstrated the same predictive validity properties (Holbrook, White, Heyman, \& Wojcicki, 2013). Despite having demonstrated good content and 
predictive validity, at least in white samples, the IIFAS lacks face validity, as the measure may appear to be assessing knowledge rather than attitudes. See Appendix D.

Brief Breastfeeding Attitudes Scale (BBAS; Lawton, Ashley, Dawson, Waiblinger, \& Conner, 2012). Given that the present study's theoretical rationale is grounded in the TPB, an additional measure that more specifically assesses dimensions of the TPB and breastfeeding was included. When measuring attitudes, Ajzen and Fishbein (2005) recommend assessing both instrumental (e.g., costs and benefits) and affective (e.g., emotional responses to a certain behavior) aspects of attitudes. The BBAS is based on a four item, semantic differential measure by Lawton and colleagues (2012), which consists of three "affective" items about breastfeeding, and one "instrumental" item about breastfeeding. In the original publication, all four items were based on a response to the stem, "For me to breastfeed my baby for the first six months would be..." (pp. 860). For the present study, the stem was revised to improve the operationalization of breastfeeding behavior to read, "For me to feed my baby only breastmilk for the first six months of the baby's life would be..." The three affective attitudes semantic differential items included “unpleasant - pleasant," "unenjoyable - enjoyable," and "inconvenient-convenient," and the instrumental item was "unhealthy - healthy." All items were based on a five-point scale, with higher scores reflecting endorsement of more positive attitudes, and total scores were calculated by summing item scores. Thus, total scores ranged from 5 to 20 . The present study utilized total scores in the analyses. In Lawton and colleague's (2012) original study, the affective items had excellent internal consistency (Cronbach $\alpha=.84)$. Affective attitudes were correlated with intention to breastfeed $(r=.60)$, ever breastfeeding $(r=.35)$, breastfeeding for six months $(r=$ $.28)$, and instrumental attitude $(r=.46)$. The instrumental attitude item was associated with 
intention to breastfeed $(r=.46)$. The BBAS also contains more face validity than the IIFAS. Author permission was granted to use this scale. See Appendix E.

Infant Feeding Knowledge Form (IFKF; Kavanagh, Lou, Nicklas, Habibi, \& Murphy, 2012). The IFKF is a self-report measure consisting of 12 statements about infant feeding. Participants read the statements and indicate whether they agree or disagree with each statement. Some of the statements are accurate and evidence-based, while others are inaccurate. Total scores are derived from summing the total correctly-answered items, and range from 0 to 12 . Higher scores indicate higher total knowledge (Kavanagh et al., 2012). The present study utilized total scores in the analyses. Kavanagh and colleagues (2012) developed this measure using four items from Giles, Connor, McClenahan, and Mallet (2010) and eight items from Tarrant and Dodgson (2007). In Kavanagh et al's (2012) original study, using an undergraduate sample, the internal consistency of the knowledge questionnaire was high (Cronbach $\alpha=.93$ ). Scores ranged from 3 to 12 , with a mean of 8.9. Scores on the IFKF were associated with scores on the IIFAS in Kavanagh et al's (2012) study, $r=.43$. Author permission was granted to use this scale. See Appendix F.

Knowledge of Infant Development Inventory (KIDI; MacPhee, 1981). The KIDI is a 75-item self-report measure designed to assess parents' knowledge of infant developmental milestones and parenting practices. The KIDI contains four subscales (though not based on factor analysis): Norms and Milestones, Principles/Facts, Parenting, and Health and Safety. Participants are presented with a variety of statements about different skills and behaviors of infants at particular ages (e.g., "Sit up on her own at six months of age"). The participants then select whether they agree or disagree with the statement. MacPhee (1981) provided an answer key in the test manual. The original 75-item measure contains excellent internal consistency 
(Cronbach $\alpha=.82$ ), Guttman split-half reliability coefficient of .85, and two-week test-retest reliability of $r=.91$.

Validity of the KIDI was established in several ways. Content validity was verified through asking pediatricians to select questions about infant development that they were most commonly asked by parents. Convergent validity was established by conducting correlational analyses among scores on the KIDI and self-reported amount of experience with infants (e.g., parenting older children, babysitting, $r=.25$ ) and infant-related education (e.g., course in child psychology, $r=.21)$. The KIDI was found to contain some educational and social class biases. although the scale was estimated to have a $6^{\text {th }}$ to $7^{\text {th }}$ grade reading level, KIDI scores were still significantly correlated with respondent education level, $r=.58$. Similarly, scores on the KIDI were positively associated with income; compared to low-SES women, "middle-class" women scored significantly higher on the KIDI.

Although the original measure is 75 items, the present study utilized only the "Norms and Milestones" subscale, which contains 20 items about cognitive, perceptual, social, and motor milestones. MacPhee (1981) stated that although this subscale was not derived from factor analysis, it could nonetheless be used as a standalone measure. The selected items assess parent knowledge of normal developmental milestones from birth through 15 months, to reflect the content covered in the psychoeducation intervention. Selections of items from the KIDI have been successfully used in other studies. Elliott (2007) utilized 19 of the 20 items in the current study, and found a KR-20 coefficient of .44. Huang, Caughy, Genebro, and Miller (2005) used 17 of the 20 items in the current study and found Cronbach's alpha $=.70$, as well as positive associations with maternal education, parity, income, and maternal depressive symptoms. Scores are determined by calculating the percentage of correct total answers. Higher scores indicate 
more accurate knowledge of infant development. The KIDI is not included in the Appendix due to copyright restrictions. The purpose of including this measure was to assess the effectiveness of the psychoeducation intervention, as well as to decrease the transparency of study aims.

Perinatal Anxiety Screening Scale (PASS; Sommerville et al., 2014). Perinatal anxiety was included in the present study as a covariate, as it is a common experience (Britton, 2005, 2007), and there is some evidence to suggest that high levels of perinatal anxiety are associated with decreased breastfeeding (Fallon, Bennett, \& Harrold, 2016). Unlike measurement of anxiety in the general population, measurement of perinatal anxiety must be sensitive to potential overlap between anxiety symptoms and experiences common to the perinatal period, such as fatigue, poor sleep, and irritability (Matthey \& Ross-Hamid, 2011). The PASS is a 31-item selfreport measure that assesses several domains of perinatal anxiety, including acute anxiety, general worry, and social anxiety (Sommerville et al., 2014). The measure was validated on pregnant and postpartum women (pregnancy through six months postpartum), and items are sensitive to the overlapping nature of anxiety and postpartum experiences. For example, women are asked to rate how often they have difficulty sleeping, "even when [they] have the chance to sleep" (Sommerville et al., 2014, p. 451). This sleep item considers the fact that for many postpartum women, sleep may be difficult because of infant awakenings, and not necessarily because of anxiety.

In terms of reliability, internal consistency for the entire scale was excellent $(\alpha=.96)$, and prenatal to postpartum test-retest reliability was adequate $(r=.74)$. The PASS demonstrated adequate convergent validity via significant correlations with all subscales of the Depression Anxiety, and Stress Scale (Depression subscale, $r=.77$; Anxiety subscale, $r=.78$; Stress subscale, $r=.92)$, the anxiety subscale of the Edinburgh Postpartum Depression Scale $(r=.82)$, 
the Beck Depression Inventory $(r=.81)$, and both subscales of the State Trait Anxiety Inventory (State subscale, $r=.75$, Trait subscale, $r=.83$ ).

Regarding screening accuracy, the PASS cutoff score of 26 correctly identified $68 \%$ of participants with anxiety disorders, which was more accurate than the more typically-used Edinburgh Postpartum Depression Scale-Anxiety and the State-Trait Anxiety Inventory - State subscale (Sommerville et al., 2014). Further, scores of 0-21, 22-41, and 42-93 represent the classifications of "mild," "moderate," and "severe" anxiety, respectively (Sommerville et al., 2015). The present study utilized total PASS scores only (not the subscales). The PASS is not included in the Appendix due to copyright restrictions.

Change rulers. These rulers measure the importance and confidence associated with a given behavior change on a scale of 0 (i.e., not at all important/not at all confident) to 10 (i.e., extremely important/extremely confident). These rulers have been used in a variety of MI interventions to evoke change talk (e.g., Butler et al., 1999; LaBrie, Quinlan, Schiffman, \& Earleywine, 2005). The change rulers were administered when appropriate during the MI session. See Appendix G.

\section{Post-intervention intentions, perceived behavioral control and importance.}

Following the assigned intervention, all participants answered questions about their infant feeding intentions, perceived behavioral control of this plan, and perceived importance of this plan. See Appendix H. The participants in the psychoeducation group also responded to several "sham" questions about what they learned from the intervention. The purpose of adding these "sham" questions was to blind the purpose of these post-intervention items, as well as for treatment integrity reasons, as later described. 
Postpartum interview. The postpartum interview was administered by phone approximately one month after the woman's reported due date. The interview was developed for the purposes of this study, but most of the items were derived from the CDC's Infant Feeding Practices Study II (Fein et al, 2008). The postpartum interview allowed recording of participant and baby health status (e.g., baby birth weight and length, current medications and medical problems), birthing experience (e.g., type of delivery, in-hospital breastfeeding support), and current breastfeeding status (Labbok \& Krasovec, 1990; Labbok \& Starling, 2012). See Appendix I.

\section{Procedure}

Recruitment, screening, and enrollment. Participants were recruited through social media advertisements, flyers, word of mouth, and clinics in Morgantown, WV, and surrounding areas. Advertisements included a study email address and phone number. Potentially eligible participants also were approached at WVU's Obstetrics and Gynecology and Family Medicine Clinics; the Morgantown Women, Infants and Children (WIC) office; and the WVU Chestnut Ridge Center's Comprehensive Opioid Addiction Treatment program. ${ }^{2}$ See Table 2 for a breakdown of recruitment sources.

\footnotetext{
${ }^{2}$ The WVU Chestnut Ridge Center Comprehensive Opioid Addiction Treatment offers intensive outpatient treatment of opioid use disorders, with specialized groups for perinatal women. Treatment combines medication-assisted therapy (buprenorphine) and group psychotherapy. Women attending these groups have maintained varying lengths of sobriety from substances. Women in this program taking buprenorphine (suboxone) as prescribed and who denied use of other substances were eligible for the present study, as buprenorphine is considered safe during breastfeeding (Graves, Turner, Nader, \& Sinha, 2016; Jansson et al., 2016; Tsai \& Doan, 2016). Further, breastfeeding is encouraged among women receiving medication-assisted treatment for opioid use disorders to reduce symptoms of neonatal abstinence syndrome (Graves et al., 2016).
} 
At the time of recruitment, participants were asked a series of screening questions to determine eligibility, including having access to a phone. See Appendix J. If the participant met inclusion criteria and agreed to participate, a date, time, and location were arranged for participation. The study procedures took place at the Quin Curtis Center for Psychological Services at West Virginia University (WVU), community locations, and participant homes. Breastfeeding promotional interventions have been successfully delivered in women's homes in the past (Napoli et al., 2004). See Table 2 for a breakdown of locations where the study took place, and Table 3 for a depiction of participants' counties and states of residence. Participants with older children were offered childcare services for the duration of the study session.

Each participant was assigned a unique study ID number that was associated with her name. The Principal Investigator (PI) maintained a participant logbook linking participant names with study ID numbers. See Appendix K. The participant logbook was kept in a locked office in the Anxiety, Psychophysiology, and Pain Research Lab in the Life Sciences Building at WVU, separate from folders of de-identified data. A contact log was maintained to record all interactions with the participant. See Appendix L. A refusal/ineligibility logbook was used to record non-participants' reason(s) for refusal and/or ineligibility. See Appendix M. No identifying information was collected for women who did not consent to participate. The purpose of the refusal logbook was to track reasons for not wanting to participate in this study and reasons for ineligibility. See Figure 1 for the study's CONSORT diagram.

Randomization. Following consent procedures, participants were randomized into the MI or psychoeducation group. As is recommended for small to medium clinical trials with the potential for imbalance of covariates between groups, the covariate adaptive randomization method was selected (Rosenberger \& Lachin, 2016). Per procedures described by Rosenberger 
and Lachin, (2016) and Suresh (2011), the first 15\% of participants ( $n=12)$ were randomly assigned to a group using the simple randomization method (Suresh, 2011), with numbers generated by an online randomizer and then entered into a spreadsheet (Urbaniak \& Plous, 2013). Thereafter, covariate adaptive randomization was used to match participants in groups based on certainty of exclusive breastfeeding, therapist (A, B), and parity (primiparous, multiparous). See Appendix $\mathrm{N}$ for item assessing certainty of exclusive breastfeeding. A spreadsheet was used to facilitate the matching and randomization process. Per single-blind procedures, participants were not informed of their group assignment.

Study therapists and supervision activities. In-person sessions and follow-up phone calls were distributed across two therapists, one of whom also was PI (SHA). Both study therapists had Master's Degrees in Clinical Psychology, as well as intermediate and advanced training in MI ( $\geq 20$ hours). This duration of MI training is commensurate with and/or exceeds the training possessed by therapists in other studies (Forsberg, Forsberg, Lindqvist, \& Helgason, 2010; Madson, Loignon, \& Lane, 2009). Daniel W. McNeil, PhD, licensed psychologist and Motivational Interviewing Network of Trainers (MINT) trainer, supervised all sessions. Content of supervision included video review and bi-weekly in-person supervision, in which Dr. McNeil provided specific feedback to both therapists regarding in-session performance. All supervision sessions were 60 minutes in duration.

Treatment integrity procedures. Both the MI and psychoeducation sessions were video recorded to ensure treatment integrity. Approximately $20 \%$ of the MI and psychoeducation sessions were then independently reviewed by two coders, who were both undergraduate research assistants. Standardized checklists were used to evaluate the presence of target content areas for the MI and psychoeducation sessions. See Appendix O. Coders were trained by the 
principal investigator (PI-Sarah H. Addicks) on procedures for reviewing the videos. The coders were instructed to check a box next to each content area when it was discussed in the session, and to record the time on the video where it occurred, as well as a few words to describe the specific interaction. The two coders viewed one video with the PI and completed the checklist together. Next, each coder independently reviewed the same video, and the results of these were reviewed with the PI, and judged to be sufficiently in agreement. Next, coders were randomly assigned eight digitally recorded sessions to watch and independently code. Each coder viewed four psychoeducation videos (two by Therapist A and two by Therapist B), and four MI videos (two by Therapist A and two by Therapist B).

Following the intervention, participants in the psychoeducation group were asked several "sham" questions to further blind them to the purpose of the study. Full results of these sham items can be found in Appendix P. Breastfeeding education was included in the psychoeducation intervention to enhance the credibility of the control group, but breastfeeding education made up less than $10 \%$ of the total intervention. One of the "sham" items asked participants to identify three new things they learned from the psychoeducation session, which was used to measure the "salience" of the breastfeeding education portion of the psychoeducation session.

Prenatal session. At the start of this session, the researcher reviewed eligibility criteria with the participant to ensure that the participant indeed qualified for the study. See Appendix J. Participants were given an overview of the study, and the researcher carefully reviewed the consent form with them. The participant was given sufficient time to read and sign the consent form. Participants were provided an opportunity, and encouraged to, ask questions, which were addressed by the researcher. 
Following consent procedures, all participants completed the prenatal interview. After this interview, participants completed five self-report measures, which include the IFKF, PASS, IIFAS, KIDI, and the BBAS, in that order. The order of these five measures was selected to obtain a non-contaminated measure of infant feeding knowledge, as the attitudes scale may have provided information to the participant that could alter responding on the knowledge form. In addition, the PASS and KIDI, which are not focused on infant feeding, served as distractors between the three feeding measures. Next, participants completed their randomly assigned intervention (i.e., MI or psychoeducation).

Prenatal Motivational Interviewing group. Participants assigned to the MI group received an approximately $45( \pm 5)$ minute intervention provided by one of the two therapists. The MI sessions were patient-centered and conversational in style, consistent with the spirit of MI (Miller \& Rollnick, 2013). As suggested by Steinberg and Miller (2015), a "Bubble Sheet" of possible topics related to breastfeeding was provided to each participant, and she was encouraged to lead the conversation by selecting topics from the sheet that interested her. See Appendix Q. A sample MI protocol is included in Appendix R. The overall structure of the protocol was adapted from a dissertation by Martins (2008) and Wilhelm et al. (2006).

Prenatal psychoeducation group. Participants who were assigned to participate in the psychoeducation group session received approximately $45( \pm 5)$ minutes of psychoeducation on typical developmental stages and infant feeding methods. The psychoeducation was provided by the same two therapists as the MI sessions. The psychoeducation group session protocol is provided in Appendix S.

Concluding procedures. After completing the assigned intervention, all participants repeated the IFKF, KIDI, and IIFAS, and BBAS, as well as the post-intervention infant feeding 
intentions, perceived behavioral control, and importance items. Participants completed the payment receipt, and a form with schedule availability and multiple modes of contact information for the purposes of planning for the follow-up phone call. See Appendix T and Appendix $U$ for the payment receipt and phone call planning form, respectively. At the end of the session, all participants received a small gift (e.g., lotion, baby toy).

Postpartum phone call. Approximately one month after the participant's self-reported due date, the researcher attempted to contact the participant to administer the postpartum interview. A maximum of ten attempts were made to contact the participant, over the course of 14 days following the participant's self-reported due date. If the researcher was unable to contact the woman after approximately ten attempts and exhausting all available contact methods (e.g., text, phone call, email), the participant was considered "lost to follow up." Once the participant completed the interview, the researcher confirmed her mailing address and mailed her a thank you note and a $\$ 40$ gift card to Wal-Mart, as outlined in the consent procedures.

\section{Results}

\section{Feasibility Assessment}

Intervention feasibility typically is evaluated across several domains. The present feasibility assessment focused on intervention demand, positive and negative effects on participants, attrition, factors affecting implementation, and resources needed to complete the project (Bowen et al., 2009).

Demand. Bowen et al. (2009) suggested that demand for an intervention can be measured by assessing the degree to which an intervention is likely to be, or is actually, used. In the present study, demand was assessed by participation rate, which was calculated by dividing the number of study participants by the number of study-eligible individuals who were actively 
recruited for the study (Harden et al., 2015). Of the 165 women who were eligible for the study, $102(61.8 \%)$ agreed to participate and subsequently scheduled an appointment. Of those women scheduled for an appointment, 81 (49.1\% of those who were initially eligible) participated in the study. To interpret this study's participation rate in the context of other research, the seven existing articles (summarized in the Introduction) on MI and breastfeeding were examined. Of those seven studies, two provided participation rates: Elliott-Rudder et al. (2014) reported a 17.6\% participation rate, while Tuthill et al. (2017) demonstrated a 98.6\% participation rate. More broadly speaking, a systematic review assessing participation rates across 82 behavioral interventions targeting health behavior change (e.g., diabetes self-management, smoking cessation) demonstrated a mean 49\% $(S D=25.0 \%)$ participation rate (Harden et al., 2015). The current study's participation rate of $49.1 \%$, then, was quite typical.

Positive and negative effects on participants. No iatrogenic effects associated with participation in this study were observed or reported by the participants. Although the study did not include a validated measure of treatment acceptability (e.g., Treatment Acceptability Questionnaire; Hunsley, 1992), anecdotal evidence (i.e., verbal/written reports directly from participant or via referral source) suggested that participants found both the MI and psychoeducation interventions to be positive experiences, describing the sessions as both "informative" and "enjoyable." One participant spontaneously emailed the PI about two months after she had completed study, and expressed her satisfaction with her breastfeeding experience and commented on the study. See Appendix V.

Practicality of intervention activities. Most participants had no difficulty completing the in-person and postpartum phone call procedures. A few factors, however, made study procedures difficult or impractical for some participants. First, the reading level of some of the 
self-report measures was too high for some participants, making completion of these measures burdensome. This high reading burden was particularly evident among women with lower levels of education (e.g., those with only a high school degree or less). Second, a few women with lower education levels seemed to have more difficulty sustaining conversation in the MI session, and often, the therapist had to rely more heavily on open-ended questions than reflections to elicit change talk. Still, all but one participant completed the entire \pm 45 -minute intervention; in this case, the participant spent most of the allotted time (2 hours) completing the self-report measures and prenatal interview, leaving little time for the intervention. Third, although none of the participants complained about the length of the intervention, behavioral indications of participation fatigue (e.g., averting gaze to check the clock, shortening verbal responses, repeating topics already covered earlier in the session, and shifting and fidgeting in the chair) were observed towards the ends of both the MI and psychoeducation interventions. While the \pm 45 -minute intervention length was chosen to optimize the MI dosage, it is possible that for some women, a shorter intervention may have been more practical and acceptable. Finally, the presence of other people (e.g., family members) and pets during some home visits caused disruptions to the overall flow of the protocols, but no major protocol deviations occurred.

Factors affecting implementation. Overall, the study was successfully executed. A total of 81 women was enrolled in the study over a period of eight months (September 2016 April 2017), and the entire data collection period, including postpartum phone calls, spanned 12 months (September 2016 - September 2017). Many factors facilitated efficient implementation of this study, the strongest of those being the utilization of two therapists, and both therapists having access to a MINT member and licensed psychologist available to provide MI supervision and consultation. Additionally, developing working relationships with community healthcare 
providers (e.g., midwives, obstetricians) facilitated recruitment, as did investing in targeted advertising through "Facebook Ads." Importantly, allowing the participant the option for an inhome visit minimized transportation barriers. The "Bubble Sheet" (included in Appendix Q) was useful for gently guiding conversation topics while also preserving participant autonomy.

On the flip side, there also were factors that impeded implementation. First, the logistics associated with therapist transportation to remote locations to meet with study participants was a significant barrier. A substantial number $(n=20)$ of interested and eligible women did not participate due to the therapists not having enough time to travel, the brief period of study eligibility (i.e., third trimester), and travel costs. This logistical issue could be remedied in the future by utilizing therapists with more schedule flexibility, partnering with organizations in remote locations for assistance with recruitment and scheduling, and additional funds. Another factor that hindered enrollment was the delay between first contact with a potential participant and time of study eligibility. Many women contacted the researcher before they entered their third trimester of pregnancy. In these cases, the researcher screened the participant and asked her if she was willing to be re-contacted after she entered her third trimester. In most cases, by the time the participant was eligible, she did not respond to calls or emails from study personnel.

Attrition. All but two of the enrolled participants completed the follow-up phone call, which translated to a completion rate of $97.5 \%$. This completion rate was equal to or higher than all the other MI and breastfeeding studies' completion rates, which ranged from $66.0 \%$ (Wilhelm et al., 2015) to $97.6 \%$ (Elliott-Rudder et al., 2014).

Resources needed for study completion. The primary resources required for this study were personnel time (i.e., work hours) and money. Each participant demanded approximately 4 hours and 15 minutes of personnel time, which was distributed across four undergraduate 
research assistants, two therapists, and the supervising psychologist. ${ }^{3}$ The total study cost was approximately \$5800, which included MI training fees, participant compensation, Facebook advertising, supplies, travel costs, and modest compensation for Therapist B.

\section{Quantitative Outcomes}

The data analysis took place in five stages: (a) preliminary examination of data, (b) determination of the psychometric properties of the IFKF, IIFAS, BBAS, KIDI, and PASS, (c) comparison of groups at baseline and at one month postpartum, (d) treatment integrity analysis, and (e) analyses addressing the research questions. PASW Statistics version 23.0 was used for all statistical analyses (IBM Corp., 2012).

Preliminary examination of data. All data were entered by three undergraduate research assistants and the PI. Approximately $10 \%$ of the data were independently checked for accuracy. Patterns of missing values were examined. None of the participants were missing more than $5 \%$ of data for the dependent variables. Similarly, no more than $5 \%$ of data were missing for any of the dependent variables, as shown in Table 4. Due to the small proportion of missing data, and the relatively small sample size, no imputations were made.

Per the assumptions of a mixed design ANOVA, data were inspected for normal distributions, homogeneity of variance, and univariate, bivariate, and multivariate outliers (Tabachnik \& Fidell, 2013). Through statistical verification and inspection of graphical representations of data, it was determined that all but three of the dependent variables were

\footnotetext{
${ }^{3}$ Breakdown of work hours associated with each participant is as follows: 30 minutes for recruitment, 15 minutes for screening and scheduling, 30 minutes for travel to participant home, 120 minutes for the in-person session, 30 minutes for training, consultation, and supervision with the supervising psychologist, and 30 minutes for the follow-up phone call; total $=4$ hours and 15 minutes.
} 
normally distributed. The variable of pre-intervention importance ("How important is it to you that you breastfeed your baby until he/she is months old?" asked prior to intervention) was non-normally distributed, with skewness $-1.84(S E=0.27)$ and kurtosis $4.90(S E=0.53)$. Similarly, the variable of post-intervention importance was non-normally distributed, with skewness -2.02 $(S E=0.27)$ and kurtosis $4.06(S E=0.54)$. Next, the post-intervention measure of infant feeding knowledge (post-intervention IFKF) was leptokurtic with skewness -1.84 (SE = $0.27)$ and kurtosis $4.90(S E=0.53)$. Following recommendations of Tabachnik and Fidell (2013), these three variables were subjected to square root transformations. After these transformations, all three variables were judged to have adequate normality, with skewness and kurtosis values $<2$. For the sake of ease of interpretation (particularly in post-hoc analyses), the original, non-transformed post-intervention IFKF variable was used in the presentation of values. See Table 5 for original and transformed skewness and kurtosis values. Two variables had extreme outliers, as defined by z-scores greater than \pm 3.29 (Tabachnick \& Fiddell, 2013). Income had two extreme outliers, and intended breastfeeding duration measured at one month postpartum had one extreme outlier. These cases were assigned raw scores one unit larger than the next most extreme score in the sample (Tabachnick \& Fiddell, 2013).

Reliability of self-report instruments. Internal consistency scores were calculated for each of the self-report measures and, when available, scores were compared to published norms. There are several published guidelines about the minimum Cronbach's alpha level required for adequate internal consistency, ranging from $\alpha=.70$ (Nunnally \& Bernstein, 1994) to $\alpha=.95$ (Bland \& Altman, 1997). In general, lower Cronbach's alpha scores can be considered adequate for research purposes, as opposed to clinical ones (Bland \& Altman, 1997). As such, the standard of $\alpha=.70$ was used as a benchmark for adequate internal consistency. The IIFAS and 
the PASS reached adequate internal consistency, and the BBAS nearly reached it. The pre- and post-intervention Cronbach's alpha coefficients for the IIFAS were . 75 and .77, respectively. These values are consistent with published norms (e.g., $\alpha=.85$; Mora et al., 1999). For the PASS, which was used only in baseline comparisons, Cronbach's alpha was .94-nearly identical to the internal consistency score reported by the developing authors $(\alpha=.96$; Sommerville et al., 2014). Cronbach's alpha for the pre- and post- intervention measurements of the BBAS were .64 and .67, respectively. When Item \#5 (instrumental attitudes-rate breastfeeding for six months as "healthy" versus "unhealthy" on a five-point Likert scale) was removed from analysis, as it was in the original published instrument, Cronbach's alpha for preand post- intervention measurements increased to .68 and 69 , respectively. The published internal consistency for the first 4 items of the BBAS was $\alpha=.84$ (Lawton et al., 2012).

The IFKF and KIDI measures had inadequate internal consistency scores. Cronbach's alphas for pre- and post-intervention IFKF were .49 and .57 , respectively. Still, the alpha levels for the current sample's IFKF were higher than those published in the original scale development study $(\alpha=.35$; Kavanagh et al., 2012). Similarly, the pre- and post-intervention KIDI internal consistency scores were $\alpha=.24$ and $\alpha=.50$, respectively, which, at least for the postintervention alpha level, is consistent with published data $(\alpha=.46$; MacPhee, 1981). Not surprisingly, the IIFAS and BBAS measures—-both of which assess attitudes towards breastfeeding — were moderately and positively correlated with each other at both pre- $(r=.43)$ and post- intervention $(r=.42)$. See Table 6 for correlations among self-report measures.

Baseline analysis. A series of $t$-tests (for continuous variables) and chi-square tests (for categorical variables) was conducted to determine whether the MI and psychoeducation groups differed on any session-related, demographic, health, or psychosocial variables. There were no 
baseline differences on any of the covariates. See Tables 7-9 for means, standard deviations, and statistical results for all baseline analyses. See Appendix W for supplementary tables showing participants' medical problems, medications, and pregnancy complications.

During randomization, participants were matched on the variable: "certainty of exclusive breastfeeding for six months" using the covariate adaptive randomization method (Rosenberger \& Lachin, 2016). Accordingly, the two groups did not differ on these scores. On a scale of -10 to +10 , the overall mean "certainty" score was $6.06(S D=4.93)$; the MI group's mean was 6.42 $(S D=4.84)$, and the psychoeducation group mean was $5.70(S D=4.91), t(79)=-0.66, p=.512$. The MI and psychoeducation groups did not differ in terms of length of contact during the sessions. The average MI session lasted 41.44 minutes $(S D=6.71)$, and the psychoeducation session averaged 41.33 minutes $(S D=4.22), t(79)=-0.91, p=.928$.

The two groups differed significantly on location of the in-person session, which was chosen by the participant, $\chi^{2}(2, N=81)=11.1, p=.004$. Over half $(n=24 ; 60.0 \%)$ of the psychoeducation sessions took place in participants' homes, whereas only about one-third $(n=$ $14 ; 34.2 \%$ ) of the MI sessions occurred in participant homes. The remainder of the psychoeducation sessions took place at the Quin Curtis Center for Psychological Services at WVU $(n=10 ; 25.0 \%)$ or in "other" locations $(n=6 ; 15.0 \%)$, such as a private room at the participant's place of work. Over half of the MI sessions took place at the Quin Curtis Center for Psychological Services at WVU $(n=25 ; 61.0 \%)$, and only two (5.0\%) took place at "other" locations. This association likely is idiosyncratic because group assignment occurred after location had already been agreed upon, and study procedures already had begun.

Groups also were compared at baseline on the bases of the dependent variables. See Table 11 for a list of means and results of $t$-tests and chi-squares comparing groups at baseline. 
The groups did not differ significantly on intention to exclusively breastfeed, duration of intended breastfeeding, perceived behavioral control, or importance of breastfeeding. Additionally, the groups did not differ significantly on the bases of pre-intervention IFKF, IIFAS, BBAS, or KIDI scores.

Baseline comparisons by therapist. Therapist A conducted 25 (60.0\%) MI sessions, and $26(63.4 \%)$ psychoeducation sessions. Therapist B conducted 15 (37.5\%) MI sessions and 15 $(36.6 \%)$ psychoeducation sessions. The therapist variable was not associated with group assignment, $\chi^{2}(1, N=81)=0.007, p=1.00$. Therapist A conducted significantly longer sessions in minutes $(M=43.06, S D=5.58)$ than Therapist $\mathrm{B}(M=38.53, S D=4.36), t(79)=4.06, p<$ .001 .

Group comparisons at one month postpartum. Information relating to the participant's labor and delivery, baby's health, and other psychosocial factors was elicited in the postpartum phone call. A series of $t$-tests and chi-square tests were conducted to illuminate any significant postpartum between-group differences that could impact the interpretation of results. The only significant difference was that women in the MI group were more likely to be giving their babies Vitamin D supplements. A larger proportion of women in the psychoeducation group had babies admitted to the NICU $(n=6)$ than those in the MI group $(n=1)$, but this difference was not statistically significant. See Table 10.

Treatment integrity analysis. The sessions were found to have excellent treatment integrity. In all but one of the videos, $100.0 \%$ of the target content areas were discussed in the sessions. The content area "AAP Breastfeeding Guidelines" was not covered in one of Therapist B's psychoeducation sessions. Of these eight randomly selected videos, content area coverage was $\geq 96.9 \%$. Further, only 4 of 93 (4.3\%) "sham" responses mentioned breastfeeding, 
suggesting that the breastfeeding education was not a particularly salient topic in the psychoeducation intervention.

Analyses for research questions. For each research question, tests were first conducted to determine if therapist (A, B) or parity (primiparous, multiparous) impacted findings. If these variables did not affect findings, then they were left out of final analyses for the sake of parsimony. Group (MI, psychoeducation) was the between-subjects factor for all repeated measures ANOVAs, and time (pre-intervention, post-intervention) was the within-subjects factor. Huynh-Feldt corrections were used to account for violations of the sphericity assumption (Huynh \& Feldt, 1976). Tukey’s Honestly Significant Difference was used to examine mean differences (Abdi \& Williams, 2010). For all tests, $p<.05$ was used to demonstrate statistical significance.

Research question 1: Intention. At pre- and post-intervention, participants selected their intended infant feeding method (only breastmilk, mix of breastmilk and formula, only formula, or undecided). A chi-square test revealed that group was not associated with a change in intended infant feeding method, $\chi^{2}(1, N=81)=0.99, p=.500$.

All participants were asked, "(If you do decide to breastfeed), how old do you think your baby will be when you completely stop breastfeeding?” A 2 (group: MI, psychoeducation) x 2 (time: pre-intervention, post-intervention) mixed factors ANOVA was performed on one continuous dependent variable: intended breastfeeding duration in months. Intended breastfeeding duration did not change from pre- $(M=10.65, S E=0.48)$ to post-intervention $(M=$ $10.53, S E=0.48), F(1,79)=0.72, p=.399$. The effect of group was non-significant, $F(1,79)$ $=0.17, p=.682$, as was the time $\mathrm{x}$ group interaction, $F(1,79)=0.373, p=.543$. See Figure 2 . 
Research question 2: Perceived behavioral control. A 2 (group: MI, psychoeducation) x 2 (Time: pre-intervention, post-intervention) mixed factors ANOVA was performed on one continuous variable: perceived behavioral control of breastfeeding. There was a significant main effect of time, $F(1,79)=4.26, p=.042$; partial $\eta^{2}=.05$, such that perceived behavioral control increased from pre- $(M=7.44, S E=0.26)$ to post-intervention $(M=7.67, S E=0.24)$. In addition, the main effect of group was significant, $F(1,79)=4.79, p=.032$; partial $\eta^{2}=.057$, such that scores in the MI group $(M=8.09, S E=0.34)$ were higher than those in the psychoeducation group $(M=7.03, S E=0.35)$. The interaction between time and group, however, was not significant, $F(1,79)=0.55, p=.463$. See Figure 3 .

Research question 3: Importance. A 2 (group: MI, psychoeducation) x 2 (time: preintervention, post-intervention) mixed factors ANOVA was conducted on one dependent variable: importance of breastfeeding. The ANOVA was first conducted with the transformed variables (square root), and then again with the non-transformed variables. The transformation did not impact the overall findings, so the original variables were used to report results for the sake of simplifying interpretation. The main effect of time was significant, $F(1,79)=10.88, p=$ .001 ; partial $\eta^{2}=.12$, such that scores increased from pre- $(M=8.16, S E=0.25)$ to postintervention $(M=8.50, S E=0.23)$. The main effect of group was not significant, $F(1,79)=$ $3.14, p=.080$, and neither was the time $\mathrm{x}$ group interaction, $F(1,79)=0.45, p=.506$. See Figure 4.

Research question 4: Infant feeding knowledge (IFKF). Preliminary $t$-tests revealed that participants assigned to Therapist A achieved significantly greater pre- to post-intervention gains on IFKF scores than those assigned to Therapist $\mathrm{B}, t(71.69)=2.01, p=.048$, equal variances not assumed. A 2 (group: MI, psychoeducation) x 2 (time: pre-intervention, post- 
intervention) x 2 (therapist: $A, B$ ) mixed factors ANOVA was performed on one continuous variable: IFKF score. There was a significant main effect of time, $F(1,75)=17.52, p<.001$, partial $\eta^{2}=.189$, such that scores increased from pre- $(M=10.20, S E=0.15)$ to post-intervention $(M=10.62, S E=0.13)$. The main effect of group was not significant, $F(1,75)=0.92, p=.763$, and neither was the time $\mathrm{x}$ group interaction, $F(1,75)=2.47, p=.120$, the group $\mathrm{x}$ therapist interaction, $F(1,75)=3.48, p=.059$, or the time $\mathrm{x}$ group $\mathrm{x}$ therapist interaction, $F(1,75)=3.62$, $p=.061$. See Figure 5 .

\section{Research question 5: Attitudes and perceived subjective norms (IIFAS, BBAS).}

IIFAS. Preliminary $t$-tests revealed that primiparous participants achieved significantly greater pre- to post-intervention gains in scores on the IIFAS than multiparous participants, $t(1$, 73 ) $=3.51, p=.001$, equal variances not assumed. A 2 (group: MI, psychoeducation) x 2 (time: pre-intervention, post-intervention) x 2 (parity: primiparous, multiparous) mixed factors ANOVA was performed on one continuous dependent variable: IIFAS. There was a significant main effect of time, $F(1,73)=19.88, p<.001$, partial $\eta^{2}=.214$, such that scores increased from pre- $(M=63.50, S E=0.91)$ to post-intervention $(M=66.06, S E=0.87)$. The main effect of group was not significant, $F(1,73)=1.56, p=.216$, and neither was the time $\mathrm{x}$ group interaction, $F(1,73)=1.64, p=.205$, or the group x parity interaction, $F(1,73)=0.23, p=.635$. See Figure 6. The group $\mathrm{x}$ time $\mathrm{x}$ parity interaction was significant, $F(1,73)=10.95, p=.001$. Tukey's post-hoc tests revealed that a pre-post increase in breastfeeding attitudes was significant only among primiparous women in the MI group $(p<.05)$. Significant differences were not observed among multiparous women in the MI group or primiparous or multiparous women in the psychoeducation group. See Figure 7. 
BBAS. A 2 (group: MI, psychoeducation) x 2 (time: pre-intervention, post-intervention) mixed factors ANOVA was conducted on one dependent variable: BBAS scores. The main effect of time was significant, $F(1,78)=12.32, p=.001$, partial $\eta^{2}=.136$, such that scores increased from pre- $(M=17.50, S E=0.26)$ to post-intervention $(M=17.93, S E=0.24)$. The main effect of group also was significant, $F(1,78)=4.63, p=.034$, partial $\eta^{2}=.056$, such that scores in the MI group $(M=18.23, S E=0.34)$ were significantly higher than those in the psychoeducation group $(M=16.98,17.20, S E=0.34)$. The group $\mathrm{x}$ time interaction was not significant, $F(1,78)=0.043, p=.837$. See Figure 8 .

Research question 6: Knowledge of infant development (KIDI). A 2 (group: MI, psychoeducation) x 2 (time: pre-intervention, post-intervention) mixed factors ANOVA was conducted on one dependent variable: KIDI scores. The main effect of time was significant, $F$ $(1,75)=8.98, p=.004$, partial $\eta^{2}=107$, such that scores on this measure increased from pre- $(M$ $=63.53, S E=1.23)$ to post-intervention $(M=67.19, S E=1.42)$. The main effect of group also was significant, $F(1,75)=5.64, p=.020$, partial $\eta^{2}=.070$, such that scores in the psychoeducation group $(M=58.16, S E=1.68)$ were higher than those in the MI group $(M=$ 62.56, $S E=1.66)$. The group $\mathrm{x}$ time interaction was not significant, $F(1,75)=3.02, p=.086$. See Figure 9.

Research question 7: Initiation. A chi-square test revealed that group was not associated with breastfeeding initiation, $\chi^{2}(1, N=79)=2.11, p=0.241$.

Research question 8: Infant feeding status at one month postpartum. Table 12 displays the distribution of participants in each IGAB feeding category by group. A chi-square test revealed that receiving MI was associated with a higher likelihood of "any current breastfeeding" at one month postpartum (IGAB categories: exclusive, partial, and token), $\chi^{2}(1, N=79)=4.30$, 
$p=0.040, \Phi=.233$ (small effect size). Another chi-square test revealed that group was not associated with "exclusive breastfeeding" (IGAB categories: exclusive and almost-exclusive breastfeeding) at one month postpartum, $\chi^{2}(1, N=79)=0.318, p=0.371$. Proportion of daily feedings as breastmilk did not significantly vary by group, $t(77)=-1.57, p=.122$.

Exploratory analysis. Additional analyses were conducted to determine whether group was associated with plans to continue breastfeeding past one month postpartum. Women were asked how old they thought their babies would be when they stopped breastfeeding, and their perceived behavioral control and importance of breastfeeding for this duration. There were no significant between-group differences on age of baby at time of breastfeeding cessation, $t(67)=$ 0.93, $p=.354$, perceived behavioral control of continuing breastfeeding, $t(67)=-1.25, p=$ 0.251 , or importance of continuing breastfeeding, $t(67)=-0.58, p=.564$. See Table 12 .

\section{Discussion}

This was the first study of its kind to assess the feasibility and preliminary effectiveness of a brief, single-session, prenatal MI intervention to promote breastfeeding in a sample of North Central Appalachian women. The objectives of the study were to assess feasibility, and to assess MI's effectiveness for enhancing breastfeeding outcomes. Specific outcomes of the present study were: (a) intention to breastfeed and intended breastfeeding duration, (b) perceived behavioral control and importance of breastfeeding plan, (c) infant feeding knowledge, (d) breastfeeding attitudes and perceived subjective norms, (e) knowledge of infant development, (f) breastfeeding behavior at one month postpartum (i.e., initiation, exclusive breastfeeding at one month postpartum, any current breastfeeding at one month postpartum), and (g) plans to continue breastfeeding past one month. The hypotheses were assessed among 81 pregnant women using a 2 (group: MI, psychoeducation) x 3 (time: pre-intervention, post-intervention, one month postpartum) single-blind randomized controlled trial. 


\section{Overall Findings}

The first objective of this study was to assess its feasibility. Overall, the study had adequate demand, low attrition, was well tolerated by participants, and —with a few adjustments - appeared appropriate for larger-scale dissemination and implementation. Feasibility could be improved by reducing reading level or total number of measures to reduce burden and possibility of fatigue. Factors impeding implementation, such as logistical difficulties associated with travel to remote locations, could be solved by increasing study personnel availability and having recruitment staff embedded in various remote WIC and obstetrics offices. Increasing the scope of this intervention would necessarily increase relative cost because personnel would be compensated relative to degree and experience.

The second main objective of this study was to determine the effectiveness of MI for enhancing breastfeeding outcomes. Notably, compared to the psychoeducation intervention, there was a significant effect of MI on breastfeeding attitudes among primiparous women, but not among multiparous women. In addition, at one month postpartum, women in the MI group were significantly more likely to report any current breastfeeding compared to women in the psychoeducation group. MI was not associated with changes in intentions to breastfeed or intended breastfeeding duration. Perceived behavioral control and importance, infant feeding knowledge, and perceived subjective norms increased significantly from pre- to post-intervention in both groups, however, there were no differential effects of MI. There were no between-group differences in exclusivity or proportion of feedings that were breastmilk. Exploratory analyses revealed no significant associations between group and participants' plans to continue breastfeeding past one month postpartum (duration, perceived behavioral control, and importance of continued breastfeeding plans). 


\section{Interpretation of Findings}

There was no effect of group on changes to intended feeding method or intended breastfeeding duration, contrary to Hypothesis 1 . This finding is fairly consistent with the limited existing literature. Neither Wilhelm et al. (2015) nor Tuthill et al. (2017) observed a significant association between MI and women's intention to breastfeed for six months. On the other hand, Cangöl and Şahin (2017) found that MI was associated with "stronger" intentions to breastfeed than the control group. It is possible that a ceiling effect could explain the lack of association between group and change in breastfeeding plans in the present study. At preintervention, over $90.0 \%$ of participants endorsed planning to exclusively breastfeed their babies ( $n=73,90.1 \%$ ), thus limiting the amount of change that could occur. Similarly, the average intended duration at pre-intervention was about 11 months, which is nearly consistent with national recommendations of breastfeeding for at least 12 months.

Certainly, the fact that nearly all participants endorsed plans to breastfeed for nearly a year is alone a positive finding. Formal efforts to educate and support pregnant and postpartum women in breastfeeding have exploded over the past 30 years (Jung, 2015), including the BabyFriendly Hospital Initiative and the WIC breastfeeding incentive program. These programs may help to normalize breastfeeding, making it more common for women to endorse plans to breastfeed for longer durations than in the past (Jung, 2015). The media, too, plays a dynamic role in creating breastfeeding's cultural norms. A qualitative media analysis by Foss (2017) showed that media representations of breastfeeding since 1974 have become increasingly positive and common. Thus, while the MI intervention was not associated with changes in breastfeeding plans or intended breastfeeding duration, the fact that an overwhelming majority of 
women planned to breastfeed exclusively and for nearly one year may reflect current cultural discourse that portrays breastfeeding as normal, positive, and healthy.

While perceived behavioral control of breastfeeding increased from pre-to postintervention for all participants, there were no between-group differences, thus not supporting Hypothesis 2. There is some inconsistency in the existing literature regarding the impact of MI on perceived behavioral control of breastfeeding. No effects of MI on perceived behavioral control were found at six weeks postpartum among women with HIV in South Africa (Tuthill et al., 2017), or at six months postpartum among rural white (Wilhelm et al., 2006), or rural Mexican American, women (Wilhelm et al., 2015). On the other hand, Cangöl and Şahin (2017) found that women assigned to their "Breastfeeding Motivation Program" reported higher levels of perceived behavioral control of breastfeeding at four weeks postpartum than women assigned to a control group. Notably, Cangöl and Şahin's (2017) study was the only one mentioned here that had a control group completely unrelated to breastfeeding (i.e., education on self-breast exams), and it was the only existing study with an intervention comprised of actual breastfeeding skill instruction. It is possible, then, that in Cangöl and Şahin's (2017) study, MI's impact on perceived behavioral control could be attributed to the more instructive content of the intervention, as well as to the control group not receiving any breastfeeding education. The MI intervention in the present study did not target the "how-tos" of breastfeeding, and instead focused on increasing motivation to breastfeed by drawing on the participant's existing strengths, and discussing potential barriers to breastfeeding and how to deal with them. While it was intended that the participant's perceived behavioral control would increase through these discussions, it is possible that an instructional component is necessary to achieve an increase in perceived behavioral control. 
Similarly, the MI intervention did not impact perceived importance of breastfeeding, contrary to Hypothesis 3 . This finding was somewhat surprising, as one of the overarching goals of MI (generally, and in the present study) is to strengthen clients' perceived importance of a behavior through exploration of clients' values (e.g., being healthy; Welch, Rose, \& Ernst, 2006). Still, this finding is consistent with findings of Cangöl and Şahin (2017), who observed no effect of MI on post-intervention (four to six weeks postpartum) measures of perceived importance of breastfeeding. Likewise, MI was not associated with increased infant feeding knowledge (IFKF), thus not supporting Hypothesis 4. None of the other MI and breastfeeding studies measured infant feeding knowledge, so it is impossible to interpret this null finding in the context of existing literature.

MI was not associated with an increase in breastfeeding perceived subjective norms. Interestingly, MI was associated with an increase in breastfeeding attitudes among primiparous women only. Thus, Hypothesis 5 was partially supported. Social psychology research suggests that in general, attitudes tend to become less flexible and more fixed with increased life experience (Krosnick \& Alwin, 1989; Tyler \& Schuller, 1991). By definition, multiparous women have more child-rearing experience, and also tend to be older than their primiparous peers (Shah, 2010). Additionally, existing literature suggests that the duration of breastfeeding one's first baby tends to be highly associated with the duration of breastfeeding subsequent children (Bai, Fong, \& Tarrant, 2015; Nagy, Orvos, Pal, Kovacs, \& Loveland, 2001). This decrease in openness to attitude change, combined with the high likelihood of repeating previous breastfeeding patterns, likely made it more difficult to change the attitudes of multiparous women. It is imperative to note that prenatal MI is especially beneficial for changing the 
attitudes of first-time mothers, which may also lead to positive, cascading effects on women's breastfeeding experiences with their future children.

A puzzling pattern emerged relating to perceived behavioral control, importance, infant feeding knowledge, and subjective norms. It simply cannot be ignored that while the MI intervention did enhance perceived behavioral control, importance, infant feeding knowledge, and breastfeeding subjective norms, so did the psychoeducation intervention. There are several possible explanations for this phenomenon. First, it is possible that the actual breastfeeding material in the psychoeducation group was as effective at increasing these outcomes as the MI session was. This explanation seems unlikely, though, because when participants were asked to describe what they learned in the psychoeducation group, very few mentioned breastfeedingrelated facts. Second, social desirability response bias may also have played a role in the findings, especially because the outcome measures were fairly face valid, and assessed by the same person serving as therapist. Participants may have endorsed higher levels of perceived behavioral control and importance of breastfeeding plans to "please" the interviewer, or in accordance with perceived demand characteristics of the study. It is possible that the "active ingredients" for pre- to post-intervention behavior change were not intervention-specific, but rather a product of reactivity to experimental arrangements (Kazdin, 2003). The mere act of participating in a research study about mother and baby health may have primed participants to respond more favorably to breastfeeding-related outcomes. Or, perhaps the "common factors" of psychotherapy (e.g., therapeutic alliance, empathy, and expectations of change; Wampold, 2015) overshadowed the specific effects of MI. There is substantial overlap between the skills of a good MI therapist and the skills of a good psychotherapist generally, particularly those skills relating to embodying MI spirit (e.g., acceptance, compassion). Both therapists in the present 
study had several years of supervised experience working as psychotherapists, and had therefore developed certain therapeutic styles that would have an effect during the psychoeducation sessions.

Perhaps even more puzzling was the finding that knowledge of infant development increased from pre- to post-intervention in both groups, contrary to Hypothesis 6 . The scores increased more in the psychoeducation group than in the MI group, but the difference was not statistically significant. There are a few possible explanations for this unexpected finding. First, it is possible that the material covered on the KIDI was inconsistent in depth and level of detail with the content presented in the psychoeducation session, resulting in lower than expected performance on the post-intervention KIDI in the psychoeducation group. Second, material covered in the MI group, such as developmentally-appropriate ages for introduction of solid foods, may have helped women in the MI group to perform nearly as well as those in the psychoeducation group. Third, strong testing effects (pre-test experience impacting post-test performance) may account for the increase in scores across groups (Kazdin, 2003). Finally, given that the time $\mathrm{x}$ group interaction was approaching traditional values of significance $(p=$ .086), it is possible that increased sample size would more obviously elucidate the differential effect of psychoeducation on KIDI scores.

Contrary to Hypothesis 7, women in the MI group were no more likely to initiate breastfeeding than those in the psychoeducation group. This finding was consistent with the limited body of existing work. Wilhelm et al. (2015) found no effect of MI on initiation rates, and Cangöl and Şahin (2017) found that women who received MI initiated breastfeeding earlier than those in the control group, however, they did not report actual rates of initiation. In the current study, $97.5 \%$ of the sample endorsed breastfeeding initiation, which greatly exceeds the 
U.S. national rate of $81.0 \%$ and the West Virginia and Pennsylvania rates of $64.6 \%$ and $73.3 \%$, respectively (CDC, 2016). The lack of between-group difference could be attributed to a ceiling effect, as all but two participants initiated breastfeeding. Again, this high rate of breastfeeding is positive news in and of itself, but may point to a problem with representativeness of the present sample, as later described.

Women in the MI group were more likely to report any current breastfeeding at one month postpartum, which is an exciting and positive finding. There were no between-group differences on breastfeeding exclusivity, proportion of feedings that were breastmilk, or future breastfeeding plans. Thus, Hypothesis 8 was partially supported. These results were relatively consistent with other published literature. Most of the other breastfeeding and lactation demonstrated higher rates of breastfeeding in MI groups, but no significant differences (Cangöl \& Sahin, 2017; Tuthill et al., 2017; Wilhelm et al., 2006; Wilhelm et al., 2015). One study (Elliott-Rudder et al., 2014) demonstrated significantly higher rates of exclusive breastfeeding at four months postpartum among women who had received three sessions of MI. Remarkably, the present study is the only single-session MI intervention to demonstrate a significant effect on breastfeeding behavior. From a public health perspective, a one-session intervention that makes moderate impact, but requires fewer resources, may be more ideal than a resource-heavy intervention that makes larger individual effects (Satcher \& Higginbotham, 2008).

\section{Strengths and Limitations}

This study has several limitations that threatened both internal and external validity. First, participants were recruited from a variety of sources (Facebook ads, local doctors' offices, etc.), which enhanced generalizability, but also introduced a surprising confound. It was discovered towards the end of data collection that some referring clinicians were referring to the 
study as the "breastfeeding study," rather than the less obtrusive title preferred by the researchers, the "WVU Mother and Infant Health Study." While these referring providers had only the best of intentions, and helped the study a great deal by making referrals, they may have inadvertently introduced selection bias to the study. Referring to the study as the "breastfeeding study," likely attracted more women who were already planning to breastfeed or who wanted to learn more about breastfeeding and clued them to study demands. Indeed, the proportion of women in this sample who initiated breastfeeding was much higher than those of national or regional norms.

Selection bias more generally also may have been an issue in this study and could have driven the limited statistical findings. Specifically, it is unclear whether the sample truly represented an "Appalachian” population. In many ways, the sample did indeed represent Appalachia: geographically speaking, all participants lived in Appalachian "proper," most identified as white, and many lived in poverty and in rural areas. Further, and perhaps even better evidence of the representativeness of the sample, many of the themes that emerged from the MI sessions were consistent with Appalachian cultural values, such as love of family, patriotism, mistrust of outsiders, and fatalism (Russ, 2010). Still, in other ways, the sample represented Appalachia less well; the women who participated in this study were primarily college-educated and in married or in long-term relationships, which is inconsistent with norms in the Appalachian region (Appalachian Regional Commission, 2017). While every effort was made to optimize accessibility for all eligible women, including single women with low or no income by offering childcare and in-home participation, many women still were unable to participate for various resource-related reasons. Anecdotally speaking, the time required for 
participation may have deterred some women from participating due to inflexible hourly work schedules or other family responsibilities, such as caring for an ill relative.

Much effort was put towards eliminating therapist engagement in MI-consistent behaviors (e.g., asking open-ended questions, embodying the MI "spirit") during the psychoeducation sessions, however, it is possible that this still occurred, resulting in treatment diffusion (Kazdin, 2003). While treatment integrity measures ensured consistent coverage of topics within each group, the presence of MI-consistent behaviors in the psychoeducation group were not assessed, making it difficult to determine the degree of treatment diffusion. Adding a waitlist-control group would also help to determine the extent to which simply participating in the study impacted outcomes. It is worth noting, however, that one of this study's strong points was its credible control group. Unlike some of the previously published literature (e.g., Cangöl \& Şahin, 2017), this study utilized an active control group, which served as a rigorous comparison to MI, thus reducing threats to internal validity (Kinser \& Robins, 2013).

Another limitation of the present study was the differential length of intervention sessions between therapists. Therapist A (SHA) averaged statistically significant longer sessions than Therapist B. The difference likely is attributable to Therapist A's larger "stake" in the study's success, as it was her dissertation project. The enhanced generalizability attributed to utilizing two therapists rather than only one may outweigh the threat to internal validity presented by differential session lengths by therapist. Further, differential session length by therapist did not translate to any therapist effects in the results.

Illicit drug use was assessed as part of the "screening" process for study eligibility, thereby excluding any self-identified, active substance users, but prenatal and postpartum alcohol use — both potential confounds - were not assessed. Women who consume alcohol during 
pregnancy are less likely to initiate breastfeeding, although this association has not been widely tested (Giglia, Binns, Alfonso, Scott, \& Oddy, 2008). Also, postpartum alcohol consumption is associated with earlier termination of breastfeeding (Breslow, Falk, Fein, \& Grummer-Strawn, 2007; Giglia et al., 2008).

The sample was predominantly non-Hispanic white (88.9\%), limiting the generalizability of findings to other ethnic and racial groups, however, the ethnic/racial breakdown of the sample was consistent with the demographics of Appalachia (82.5\%, non-Hispanic white; Appalachian Regional Commission, 2017) — the targeted population for this intervention. Most of the participants in the present study resided in North Central Appalachia (most of West Virginia, southern Ohio, and a few counties in southern Pennsylvania), where the population is $92.5 \%$ non-Hispanic white, thus the current sample closely reflects population racial demographics (Appalachian Regional Commission, 2017). Still, the findings of the present study may not be generalizable to women of other races and ethnicities. Compared to their white peers, African Americans, Latinas, and American Indians/Alaska Natives are more likely to face numerous upstream factors (e.g., poverty, discrimination, language or literacy barriers) that negatively affect breastfeeding outcomes (Bartick et al., 2017; Jones et al., 2015; Jung, 2015). Future work in this area would benefit from specifically gearing recruitment efforts towards racial and ethnic minorities, and culturally adapting MI breastfeeding interventions accordingly.

Participants' sexual orientation and gender identity were not assessed in the present study, which represents both a conceptual and methodological limitation. Little research acknowledges breastfeeding as an issue pertaining to LGBTQ populations, let alone addresses breastfeeding and lactation in this population (Farrow, 2014). While LGBTQ people were not barred from participating, the language used in the present study (and in most lactation research) 
assumed a cisnormative and heteronormative stance, which could be perceived as an erasure or exclusion of individuals not identifying as cisgender, heterosexual women (Farrow, 2014). More practically speaking, by not assessing sexual orientation and gender identity, it is impossible to generalize findings to LGBTQ populations.

The mean household income in the present study $(\$ 83,234)$ exceeded U.S. national (\$75,062; U.S. Census Bureau, 2017) and North Central Appalachian $(\$ 62,254)$ mean household incomes, which could limit generalizability to lower-income women (Appalachian Regional Commission, 2017). Then again, annual income in this sample ranged from $\$ 0$ to nearly $\$ 500,000$; researchers met with participants in a local motel, mobile homes, and large, luxurious homes, illustrating the wide range of socio-economic status captured in this sample.

A major strength of this study was its excellent generalizability to a variety of "realworld" settings, including participant homes and clinics. Along with the in-home visits came a myriad of sources of interruptions, including pets, family members, television and video game noises, and, in one case, major construction in the home. Still, the protocol was easily adaptable to distractions and interruptions, and no deviations occurred. Distractions and interruptions occurred in the clinic setting, too. Several women who chose to meet with the researcher at the clinic still brought along their older children, who understandably caused interruptions. While of course a "sterile" experimental environment may have maximized internal validity, it simply is not consistent with the realities of families with young children. Child attendance at parent doctors' appointments, particularly mothers, is a necessity for many families. Research has demonstrated that lack of childcare is a common barrier to prenatal appointment attendance, particularly among low-income women (Quinn, Detmer, \& Bell-Ellison, 2008). Therefore, it has been recommended that prenatal healthcare providers create a child-friendly atmosphere to allow 
for optimal appointment attendance (Quinn et al., 2008). It is a definite strength that this intervention was still feasible and effective in the face of many "real world" interruptions, attesting to its transportability to busy healthcare settings.

Finally, a larger proportion of women in control group had babies admitted to the NICU than those in the MI group. Even though this difference was not statistically significant, it is possible that it impacted findings, and therefore should be considered a limitation. Babies admitted to the NICU are less likely to receive breastmilk than their healthy peers (Flacking et al., 2005; Wight, 2015). Because group assignment occurred during the woman's pregnancy, NICU admission was an impossible variable on which to "match" participants between groups.

\section{Future Directions}

The utility of MI for improving breastfeeding outcomes has not yet been fully explored, creating abundant opportunities for future research. Future efforts should aim to simultaneously preserve intervention resource-efficiency while increasing effectiveness. Given that skill selfefficacy is essential to any health behavior change, regardless of the guiding theoretical model (Ajzen, 1991; Glanz, Rimer, \& Lewis, 2002), future interventions in this area may benefit from including breastfeeding skills trainings (e.g., positioning of baby) in combination with MI, similar to the intervention described by Cangöl and Şahin (2017). Along those lines, assigning some women to receive MI with instructive breastfeeding content and some to receive solely MI or instructive breastfeeding content would allow researchers to determine the true "added benefit" of MI over "treatment as usual" skills trainings common to many settings (e.g., WIC), as well as whether instructive content bolsters or dilutes the effects of MI.

Future researchers may also want to consider telehealth as an intervention modality that could help to reach women with transportation barriers, or adding text messages as an adjunct to 
MI interventions as virtual "booster sessions" that serve to provide encouragement, affirmation of goals, support, and information, when appropriate. Teens and young adults, the most likely target of future breastfeeding interventions, prefer text messaging over other forms of communication (Newport, 2014). Zunza, Cotton, Mbuagbaw, Lester, and Thabane (2017) recently proposed an investigation of the effectiveness of text messaging as an adjunct to MI to promote exclusive breastfeeding among women with HIV in South Africa. The authors stated that the text messages will encourage women to breastfeed exclusively, and help to troubleshoot lactation problems as they arise. Although the results of this proposed study are not yet available, it is possible that adjunctive texting or online communication to MI could be a lowresource strategy for bolstering treatment effects.

It is crucial that future breastfeeding interventions target the women who would most benefit from support. There continue to be great racial, economic, and social inequities related to access to and utilization of prenatal care (Edmonds, Mogul, \& Shea, 2015; Heaman et al., 2015) and skilled lactation care (Friesen et al., 2015), both of which perpetuate breastfeeding disparities (Lind, Perrine, Li, Scanlon, Grummer-Strawn, \& CDC, 2014). Social determinants of breastfeeding behavior include income, education, and experiences of discrimination, among others. Much of the necessary breastfeeding promotion work lies not in the intensive, individualized, behavioral interventions, but rather in the realm of large-scale public health initiatives, however, there is a history of a "bottleneck" effect when it comes to transforming "knowledge" into improved health outcomes. Pérez-Escamilla and Sellen (2015) wrote:

we have a wealth of knowledge that could be immediately put into practice to address breastfeeding inequities globally. Unfortunately, investments in breastfeeding scaling-up research that takes equity issues into consideration continues to be extremely limited. It 
is time we move forward with the design, implementation and evaluation of programs that address the breastfeeding needs of all. (p. 13)

Accordingly, expansions of this line of research should use MI to specifically target historically underserved populations, such as American Indians, African Americans, veterans and active duty service members (Pérez-Escamillo \& Sellen, 2015), LGBTQ individuals (Farrow, 2014), women with disabilities (Redshaw, Malouf, Gao, \& Gray, 2013), and lowincome women (Newhook et al., 2017), among others. Of course, cultural adaptations should be made to the MI intervention as necessary (Wilhlem et al., 2015). Another population that may achieve great benefit from MI for breastfeeding enhancement is the growing number of women receiving medication-assisted treatment of opioid use disorders (Substance Abuse and Mental Health Services Administration, 2013). Breastfeeding is recommended for women receiving medication-assisted treatment because it may reduce the occurrence of or complications related to neonatal abstinence syndrome (Graves, Turner, Nader, \& Sinha, 2016; Jansson et al., 2016; Tsai \& Doan, 2016), but breastfeeding rates are lower among women receiving medicationassisted treatment than the general population (Tsai \& Doan, 2016).

Importantly, most people identifying with any of the above-mentioned (or other) minority statuses often feel mistrust towards healthcare providers due to a history of marginalization, mistreatment, or stigmatization in medical settings (Dovidio et al., 2008; Olsen \& Sharfstein, 2014). MI, with its emphases on partnership and patient autonomy, may be more acceptable to women with histories of oppression than traditional "advice-giving" approaches.

\section{Conclusions}

To conclude, patient-centered, individualized, approaches to breastfeeding promotion, such as MI, are increasingly recognized as "best practice" by healthcare professionals (ACOG 
Committee on Obstetric Practice, 2016). The present investigation demonstrated that a TBPdriven, single-session prenatal MI intervention in the third trimester was feasible, effective in increasing breastfeeding attitudes among primiparous women, and generally effective in increasing the likelihood of breastfeeding at one month postpartum. The intervention was resource-efficient and well-received by participants, making it suitable for larger scale implementation. 


\section{References}

Abdi, H., \& Williams, L. J. (2010). Tukey’s Honestly Significant Difference (HSD) Test. In N. Salkind (Ed.), Encyclopedia of research design (pp. 1-5). Thousand Oaks, CA: Sage.

Abrahams, S. W., \& Labbok, M. H. (2009). Exploring the impact of the baby-friendly hospital Initiative on trends in exclusive breastfeeding. International Breastfeeding Journal, 4, e1-e6. doi:10.1186/1746-4358-4-11

Ajzen, I. (1988). Attitudes, personality, and behavior. Chicago, IL: Dorsey Press.

Ajzen, I. (1991). The theory of planned behavior. Organizational Behavior and Human Decision Processes, 50, 179-211.

Ajzen, I., \& Fishbein, M. (2005). The influence of attitudes on behavior. In D. Albarracín, B. T. Johnson, \& M. P. Zanna (Eds.), The handbook of attitudes (pp. 173-221). Mahwah, NJ: Erlbaum.

Alexander, J. M., Grant, A. M., \& Campbell, M. J. (1992). Randomised controlled trial of breast shells and Hoffman's exercises for inverted and non-protractile nipples. British Medical Journal, 304, 1030-1032. doi:10.1136/bmj.304.6833.1030

American Academy of Family Practice Breastfeeding Advisory Committee. (2014). Breastfeeding, family physicians supporting (position paper). Retrieved from http://www.aafp.org/about/policies/all/breastfeeding-support.html

American Academy of Pediatrics (2013). Federal support for breastfeeding. Retrieved from https://www2.aap.org/breastfeeding/files/pdf/FederalSupportforBreastfeeding Resource.pdf 
American Academy of Pediatrics Section on Breastfeeding. (2012). Policy statement:

Breastfeeding and the use of human milk. Pediatrics, 129, e827-e841. doi:10.1542/peds.2011-3552

American Academy of Pediatrics. (2001). The transfer of drugs and other chemicals into human milk. Pediatrics, 108, 776-789. doi:10.1542/peds.108.3.776

American College of Obstetrics and Gynecology Committee on Obstetric Practice. (2016). Optimizing support for breastfeeding as part of obstetric practice. Committee Opinion No. 658. American College of Obstetricians and Gynecologists. Obstetrics \& Gynecology, 127, e86-92.

American College of Obstetrics and Gynecology Committee on Underserved Women. (2011). Committee Opinion No. 473. American College of Obstetricians and Gynecologists. Obstetrics \& Gynecology, 117, 200-201.

Amir, L. H., \& Donath, S. M. (2012). Maternal diet and breastfeeding: A case for rethinking physiological explanations for breastfeeding determinants. Early Human Development, 88, 467-471. doi:10.1016/j.earlhumdev.2011.11.002

Appalachian Regional Commission. (2017). Washington, DC, USA, Retrieved from https://www.arc.gov/assets/research_reports/DataOverviewfrom2011to2015ACS.pdf

Arain, M., Campbell, M. J., Cooper, C. L., \& Lancaster, G. A. (2010). What is a pilot or feasibility study? A review of current practice and editorial policy. BMC Medical Research Methodology, 10, 67. doi:10.1186/1471-2288-10-67

Avery, M., Duckett, L., Dodgson, J., Savik, K., \& Henly, S. J. (1998). Factors associated with very early weaning among primiparas intending to breastfeed. Maternal and Child Health Journal, 2, 167-179. doi:10.1023/A:1021879227044 
Baby-Friendly USA, Inc. (2016). Guidelines and evaluation criteria for facilities seeking babyfriendly designation. Retrieved from https://www.babyfriendlyusa.org/get-started/theguidelines-evaluation-criteria

Baby-Friendly USA, Inc. (2017). Designated facilities by state. Retrieved from https://www.babyfriendlyusa.org/find-facilities/designated-facilities--by-state

Bai, D. L., Fong, D. Y. T., \& Tarrant, M. (2015). Previous breastfeeding experience and duration of any and exclusive breastfeeding among multiparous mothers. Birth, 42, 70-77. doi:10.1111/birt.12152

Bai, J., Wong, F. W., Bauman, A., \& Mohsin, M. (2002). Parity and pregnancy outcomes. American Journal of Obstetrics and Gynecology, 186, 274-278. doi:10.1067/mob.2002.119639

Baker, M., \& Milligan, K. (2008). Maternal employment, breastfeeding, and health: Evidence from maternity leave mandates. Journal of Health Economics, 27, 871-887. doi:10.3386/w13188

Balyakina, E., Fulda, K. G., Franks, S. F., Cardarelli, K. M., \& Hinkle, K. (2016). Association between healthcare provider type and intent to breastfeed among expectant mothers. Maternal and Child Health Journal, 20, 993-1000. doi:10.1007/s10995-0151884-X

Bartick, M. C., Schwarz, E. B., Green, B. D., Jegier, B. J., Reinhold, A. G., Colaizy, T. T., ... \& Stuebe, A. M. (2017). Suboptimal breastfeeding in the United States: Maternal and pediatric health outcomes and costs. Maternal \& Child Nutrition, 13, 3-6. doi:10.1111/mcn.12366 
Bartick, M. C., Stuebe, A. M., Schwarz, E. B., Luongo, C., Reinhold, A. G., \& Foster, E. M. (2013). Cost analysis of maternal disease associated with suboptimal breastfeeding. Obstetrics \& Gynecology, 122, 111-119. doi:10.1097/AOG.0b013e318297a047

Bartick, M., \& Reinhold, A. (2010). The burden of suboptimal breastfeeding in the United States: A pediatric cost analysis. Pediatrics, 125(5), e1048-e1056. doi:10.1542/peds.2009-1616

Barton, S. J. (2001). Infant feeding practices of low-income rural mothers. MCN: The American Journal of Maternal/Child Nursing, 26, 93-97.

Bass, C. A., Rodgers, M., \& Baker, H. R. (2014). Can placing a lactation consultant in the obstetric office magically increase exclusive breastfeeding rates? Journal of Obstetric, Gynecologic, \& Neonatal Nursing, 43, S52-S52. doi:10.1111/1552-6909.12352

Berlin, K., Hamel-Lambert, J., \& DeLamatre, C. (2013). Obesity and overweight status health disparities among low-income rural Appalachian preschool children. Children's Health Care, 42, 15-26. doi:10.1080/02739615.2013.753812

Berman, A. H., Forsberg, L., Durbeej, N., Källmén, H., \& Hermansson, U. (2010). Singlesession motivational interviewing for drug detoxification inpatients: effects on selfefficacy, stages of change and substance use. Substance Use \& Misuse, 45, 384-402. doi:10.3109/10826080903452488

Bland, J. M., \& Altman, D. G. (1997). Statistics notes: Cronbach's alpha. British Medical Journal, 314, 572. 
Blyth, R., Creedy, D. K., Dennis, C. L., Moyle, W., Pratt, J., \& De Vries, S. M. (2002). Effect of maternal confidence on breastfeeding duration: An application of breastfeeding selfefficacy theory. Birth, 29, 278-284. doi:10.1046/j.1523-536X.2002.00202.x

Bonuck, K. A., Trombley, M., Freeman, K., \& McKee, D. (2005). Randomized, controlled trial of a prenatal and postnatal lactation consultant intervention on duration and intensity of breastfeeding up to 12 months. Pediatrics, 116, 1413-1426. doi:10.1542/peds.20050435

Bonuck, K., Stuebe, A., Barnett, J., Labbok, M. H., Fletcher, J., \& Bernstein, P. S. (2014). Effect of primary care intervention on breastfeeding duration and intensity. American Journal of Public Health, 104, S119-S127. doi:10.2105/AJPH.2013.301360

Borrelli, B., Tooley, E. M., \& Scott-Sheldon, L. A. J. (2015). Motivational interviewing for parent-child health interventions: A systematic review and meta-analysis. Pediatric Dentistry, 37, 254-265.

Bowen, D. J., Kreuter, M., Spring, B., Cofta-Woerpel, L., Linnan, L., Weiner, D., ... \& Fernandez, M. (2009). How we design feasibility studies. American Journal of Preventive Medicine, 36, 452-457. doi:10.1016/j.amepre.2009.02.002

Bramhagen, A. C., Axelsson, I., \& Hallström, I. (2006). Mothers' experiences of feeding situations-an interview study. Journal of Clinical Nursing, 15, 29-34. doi:10.1111/j.1365-2702.2005.01242.x

Breslow, R. A., Falk, D. E., Fein, S. B., \& Grummer-Strawn, L. M. (2007). Alcohol consumption among breastfeeding women. Breastfeeding Medicine, 2, 152-157. doi:10.1089/bfm.2007.0012 
Brew, B. K., Marks, G. B., Almqvist, C., Cistulli, P. A., Webb, K., \& Marshall, N. S. (2014). Breastfeeding and snoring: a birth cohort study. PloS One, 9, e84956. doi:10.1371/ journal.pone.0084956

Britton, J. R. (2005). Pre- discharge anxiety among mothers of well newborns: Prevalence and correlates. Acta Paediatrica, 94, 1771-1776. doi:10.1111/j.1651-2227.2005.tb01852.x

Britton, J. R. (2007). Postpartum anxiety and breast feeding. The Journal of Reproductive Medicine, 52, 689-695.

Brown, A. (2014). Maternal trait personality and breastfeeding duration: The importance of confidence and social support. Journal of Advanced Nursing, 70, 587-598. doi:10.1111/jan.12219

Brown, A., Raynor, P., \& Lee, M. (2011). Healthcare professionals' and mothers' perceptions of factors that influence decisions to breastfeed or formula feed infants: A comparative study. Journal of Advanced Nursing 67, 1993-2003. doi:10.1111/j.13652648.2011.05647.x

Brown, C. R., Dodds, L., Legge, A., Bryanton, J., \& Semenic, S. (2014). Factors influencing the reasons why mothers stop breastfeeding. Canadian Journal of Public Health, 105, e17985.

Buckley, K. M., \& Charles, G. E. (2006). Benefits and challenges of transitioning preterm infants to at-breast feedings. International Breastfeeding Journal, 1, 1746-4358. doi:10.1186/1746-4358-1-13

Burke, B. L., Arkowitz, H., \& Menchola, M. (2003). The efficacy of motivational interviewing: a meta-analysis of controlled clinical trials. Journal of Consulting and Clinical Psychology, 71, 843-861. doi:10.1037/0022-006X.71.5.843 
Butler, C. C., Rollnick, S., Cohen, D., Bachmann, M., Russell, I., \& Stott, N. (1999).

Motivational consulting versus brief advice for smokers in general practice: A randomized trial. British Journal of General Practice, 49, 611- 616.

Calnen, G. (2007). Paid maternity leave and its impact on breastfeeding in the United States: an historic, economic, political, and social perspective. Breastfeeding Medicine, 2, 34-44. doi:10.1089/bfm.2006.0023

Cangöl, E., \& Şahin, N. H. (2017). The effect of a breastfeeding motivation program maintained during pregnancy on supporting breastfeeding: A randomized controlled trial. Breastfeeding Medicine, 12, 218-226. doi:10.1089/bfm.2016.0190

Carroll, K. M., Libby, B., Sheehan, J., \& Hyland, N. (2001). Motivational Interviewing to enhance treatment initiation in substance abusers: An effectiveness study. The American Journal on Addictions/American Academy of Psychiatrists in Alcoholism and Addictions, 10, 335-339. doi:10.1111/j.1521-0391.2001.tb00523.x

Cartwright, J., Atz, T., Newman, S., Mueller, M., \& Demirci, J. R. (2017). An integrative review of interventions to promote breastfeeding in the late preterm infant. Journal of Obstetric, Gynecologic \& Neonatal Nursing, 46. 347-357. doi:10.1016/j.jogn.2017.01.006

Cascaes, A. M., Bielemann, R. M., Clark, V. L., \& Barros, A. J. D. (2014). Effectiveness of motivational interviewing at improving oral health: A systematic review. Revista De Saúde Pública, 48, 142-153. doi:10.1590/S0034-8910.2014048004616

Centers for Disease Control and Prevention. (2015). Maternity practices in infant nutrition and care (mPINC) survey. Retrieved from http://www.cdc.gov/breastfeeding/data/mpinc/index.htm 
Centers for Disease Control and Prevention. (2016). Breastfeeding report card. Retrieved from http://www.cdc.gov/breastfeeding/pdf/2014breastfeedingreportcard.pdf

Chantry, C. J., Eglash, A., \& Labbok, M. (2015). ABM position on Breastfeeding—Revised 2015. Breastfeeding Medicine, 10, 407-411. doi:10.1089/bfm.2015.29012.cha

Chapman, D. J., \& Pérez-Escamilla, R. (2000). Maternal perception of the onset of lactation is a valid, public health indicator of lactogenesis stage II. The Journal of Nutrition, 130, 2972-2980.

Chaput, K. H., Nettel-Aguirre, A., Musto, R., Adair, C. E., \& Tough, S. C. (2016). Breastfeeding difficulties and supports and risk of postpartum depression in a cohort of women who have given birth in Calgary: a prospective cohort study. CMAJ Open, 4, E103. doi:10.9778/cmajo.20150009

Chen, H., Cohen, P., \& Chen, S. (2010). How big is a big odds ratio? Interpreting the magnitudes of odds ratios in epidemiological studies. Communications in Statistics-Simulation and Computation, 39, 860-864. doi:10.1080/03610911003650383

Chen, P. L., Soto-Ramírez, N., Zhang, H., \& Karmaus, W. (2017). Association between infant feeding modes and gastroesophageal reflux: A repeated measurement analysis of the infant feeding practices study II. Journal of Human Lactation, 33, 267-277. doi:10.1177/0890334416664711

Chertok, I. R. A., \& Hoover, M. L. (2009). Breastfeeding legislation in states with relatively low breastfeeding rates compared to breastfeeding legislation of other states. Journal of Nursing Law, 13, 45-53. doi:10.1891/1073-7472.13.2.45

Chertok, I. R. A., Luo, J., Culp, S., \& Mullett, M. (2011). Intent to breastfeed: A populationbased perspective. Breastfeeding Medicine, 6, 125-129. doi:10.1089/bfm.2010.0013 
Chezem, J., Friesen, C., \& Boettcher, J. (2003). Breastfeeding knowledge, breastfeeding confidence, and infant feeding plans: Effects on actual feeding practices. Journal of Obstetric, Gynecologic, and Neonatal Nursing, 32, 40-47. doi:10.1177/0884217502239799

Child Welfare Information Gateway. (2015). “State Statutes Search.” Retrieved from https://www.childwelfare.gov/topics/systemwide/laws-policies/state/

Chiou, S., Chen, L., Yeh, H., Wu, S., \& Chien, L. (2014). Early skin- to- skin contact, rooming- in, and breastfeeding: A comparison of the 2004 and 2011 national surveys in Taiwan. Birth, 41, 33-38. doi:10.1111/birt.1209

Chung, M., Raman, G., Trikalinos, T., Lau, J. U., \& Ip, S. (2008). Interventions in primary care to promote breastfeeding: An evidence review for the U.S. preventive services task force. Annals of Internal Medicine, 149, 565-582. doi:10.7326/0003-4819-149-8-20081021000009

Cohen, J. (1988). Statistical power analysis for the behavioral sciences. New York, NY: Academic Press.

Copeland, L., Merrett, L., Grant, A., Phillips, R., Tedstone, S., Sanders, J., ... \& Paranjothy, S. (2016). Mam-Kind, a novel peer support intervention of motivational interviewing for breastfeeding maintenance: a feasibility study in the UK. The Lancet, 388, S38. doi:10.1016/S0140-6736(16)32274-7

Cronin, C. (2003). First- time mothers - identifying their needs, perceptions and experiences. Journal of Clinical Nursing, 12, 260-267. doi:10.1046/j.13652702.2003.00684.x 
da Silva, C. F., Nunes, L. M., Schwartz, R., \& Giugliani, E. R. J. (2016). Effect of a probreastfeeding intervention on the maintenance of breastfeeding for 2 years or more: Randomized clinical trial with adolescent mothers and grandmothers. BMC Pregnancy and Childbirth, 16, 97. doi:10.1186/s12884-016-0878-z

Davoli, A., Broccoli, S., Bonvicini, L., Fabbri, A., Ferrari, E., D'Angelo, S., \& Rossi, P. (2013). Pediatrician-led motivational interviewing to treat overweight children: An RCT. Pediatrics, 132, E1236-E1246. doi:10.1542/peds.2013-1738

de Oliveira, M. I. C., Camacho, L. A. B., \& Tedstone, A. E. (2001). Extending breastfeeding duration through primary care: A systematic review of prenatal and postnatal interventions. Journal of Human Lactation, 17, 326-343.

De Wilde, K., Maes, L., Boudrez, H., Tency, I., Temmerman, M., \& Clays, E. (2017). Analysis of smoking cessation beliefs in pregnant smokers and ex-smokers using the Theory of Planned Behavior. Journal of Public Health, 25, 267-274. doi:10.1007/s10389-0160784-X

Dennis, C. L. (2002). Breastfeeding peer support: Maternal and volunteer perceptions from a randomized controlled trial. Birth, 29, 169-176. doi:10.1046/j.1523536X.2002.00184.X

Dennis, C. L., \& McQueen, K. (2007). Does maternal postpartum depressive symptomatology infant feeding outcomes? Acta Paediatrica, 96, 590-594. doi:10.1111/j.16512227.2007.00184.x

Dodgson, J. E., \& Tarrant, M. (2007). Outcomes of a breastfeeding educational intervention for baccalaureate nursing students. Nurse Education Today, 27, 856-867. doi:10.1016/j.nedt.2006.12.001 
Dodgson, J. E., Henly, S. J., Duckett, L., \& Tarrant, M. (2003). Theory of planned behaviorbased models for breastfeeding duration among Hong Kong mothers. Nursing Research, 52, 148-158.

Dovidio, J. F., Penner, L. A., Albrecht, T. L., Norton, W. E., Gaertner, S. L., \& Shelton, J. N. (2008). Disparities and distrust: The implications of psychological processes for understanding racial disparities in health and health care. Social Science \& Medicine, 67, 478-486. doi:10.1016/j.socscimed.2008.03.019

Dozier, A. M., Howard, C. R., Brownell, E. A., Wissler, R. N., Glantz, J. C., Ternullo, S. R.. . Lawrence, R. A. (2013). Labor epidural anesthesia, obstetric factors and breastfeeding cessation. Maternal and Child Health Journal, 17, 689-698. doi:10.1007/s10995-012$1045-4$

Dubois, L., \& Girard, M. (2003). Social determinants of initiation, duration and exclusivity of breastfeeding at the population level: The results of the longitudinal study of child development in Quebec (ELDEQ 1998-2002). Canadian Journal of Public Health, 94, 300-305. doi:10.17268/cjph.94.351

Duckett, L., Henly, S., Avery, M., Potter, S., Hills-Bonczyk, S., Hulden, R., \& Savik, K. (1998). A theory of planned behavior-based structural model for breast-feeding. Nursing Research, 47, 325-336.

Dungy, C. I., McInnes, R. J., Tappin, D. M., Wallis, A. B., \& Oprescu, F. (2008). Infant feeding attitudes and knowledge among socioeconomically disadvantaged women in Glasgow. Maternal and Child Health Journal, 12, 313-322. doi:10.1007/s10995-0070253-9 
Edmonds, B. T., Mogul, M., \& Shea, J. A. (2015). Understanding low-income African American women's expectations, preferences, and priorities in prenatal care. Family \& Community Health, 38, 149-157. doi:1.1097/FCH.0000000000000066

Einarson, A., \& Riordan, S. (2009). Smoking in pregnancy and lactation: A review of risks and cessation strategies. European Journal of Clinical Pharmacology, 65, 325-330. doi:10.1007/s00228-008-0609-0

Elliott, N. L. (2007). First-time mothers' parenting knowledge during their infants' first year: Relations with information sources, social support networks, maternal self-efficacy, and infant development. (Unpublished doctoral dissertation). West Virginia University, Morgantown, WV.

Elliott- Rudder, M., Pilotto, L., McIntyre, E., \& Ramanathan, S. (2014). Motivational interviewing improves exclusive breastfeeding in an Australian randomised controlled trial. Acta Paediatrica, 103, e11-e16. doi:10.1111/apa.12434

Else-Quest, N. M., Hyde, J. S., \& Clark, R. (2003). Breastfeeding, bonding, and the motherinfant relationship. Merrill-Palmer Quarterly, 49, 495-517. doi:10.1353/mpq.2003.0020

Emmons, K., \& Rollnick, S. (2001). Motivational interviewing in health care settings opportunities and limitations. American Journal of Preventive Medicine, 20, 68-74. doi:10.1016/S0749-3797(00)00254-3

Erickson, S. J., Gerstle, M., \& Feldstein, S. W. (2005). Brief interventions and motivational interviewing with children, adolescents, and their parents in pediatric health care settings: A review. Archives of Pediatrics \& Adolescent Medicine, 159, 1173-1180. doi:10.1001/archpedi.159.12.1173 
Ertem, I. O., Votto, N., \& Leventhal, J. M. (2001). The timing and predictors of the early termination of breastfeeding. Pediatrics, 107, 543-548. doi:10.1542/peds.107.3.543

Fair Labor Standards Act. 29 U.S.C. 201, et seq. (1938).

Fallon, V., Bennett, K. M., \& Harrold, J. A. (2016). Prenatal anxiety and infant feeding outcomes: A systematic review. Journal of Human Lactation, 32, 53-66. doi: $10.1177 / 0890334415604129$

Farrow, A. (2015). Lactation support and the LGBTQI community. Journal of Human Lactation, 31, 26-28. doi:10.1177/0890334414554928

Faul, F., Erdfelder, E., Lang, A. G., \& Buchner, A. (2007). G*Power 3: A flexible statistical power analysis program for the social, behavioral, and biomedical sciences. Behavior Research Methods, 39, 175-191.

Fein, S. B., Labiner-Wolfe, J., Shealy, K. R., Li, R., Chen, J., \& Grummer-Strawn, L. M. (2008). Infant feeding practices study II: Study methods. Pediatrics, 122, S28-S35. doi:10.1542/peds.2008-1315c

Fisher, J., \& Stocky, A. (2006). Maternal perinatal mental health and multiple births: Implications for practice. Twin Research and Human Genetics, 6, 506-513.

Flacking, R., Ewald, U., Nyqvist, K. H., \& Starrin, B. Trustful bonds: A key to "becoming a mother" and to reciprocal breastfeeding. Stories of mothers of very preterm infants at a neonatal unit. (2006). Social Science and Medicine, 62, 70-80. doi:10.1016/j.socscimed.2005.05.026.

Flower, K. B., Willoughby, M., Cadigan, R. J., Perrin, E. M., Randolph, G., \& Family Life Project Investigative Team. (2008). Understanding breastfeeding initiation and continuation in rural communities: a combined qualitative/quantitative 
approach. Maternal and Child Health Journal, 12, 402-414. doi:10.1007/s10995-0070248-6

Forsberg, L., Forsberg, L. G., Lindqvist, H., \& Helgason, A. R. (2010). Clinician acquisition and retention of Motivational Interviewing skills: a two-and-a-half-year exploratory study. Substance Abuse Treatment, Prevention, and Policy, 5, 1. doi:10.1186/1747597X- 5-8

Forster, D., McLachlan, H., Davey, M., Amir, L., Gold, L., Small, R.. . McLardie-Hore, F. (2014). Ringing up about breastfeeding: A randomised controlled trial exploring early telephone peer support for breastfeeding (RUBY) - trial protocol. BMC Pregnancy and Childbirth, 14, 177. doi:10.1186/1471-2393-14-177

Foss K.A. (2017). Breastfeeding and media: Exploring conflicting discourses that threaten public health. Cham, Switzerland: Palgrave Macmillan.

Friesen, C. A., Hormuth, L. J., Petersen, D., \& Babbitt, T. (2015). Using videoconferencing technology to provide breastfeeding support to low-income women connecting hospitalbased lactation consultants with clients receiving care at a community health center. Journal of Human Lactation, 31, 595-599. doi:10.1177/0890334415601088

Gail, C. C., \& Krell, J. K. (2014). Changing the breastfeeding conversation and our culture. Breastfeeding Medicine, 9, 53-55. doi:10.1089/bfm.2014.9994

Gantt, C. J. (2001). The theory of planned behavior and postpartum smoking relapse. Journal of Nursing Scholarship, 33, 337-341. doi:10.1111/j.1547-5069.2001.00337.x

George, L. (2005). Lack of preparedness: experiences of first time mothers. American Journal of Maternal Child Nursing, 30, 251-255. doi:10.1097/00005721-200507000-00009 
Giglia, R. C., Binns, C. W., Alfonso, H. S., Scott, J. A., \& Oddy, W. H. (2008). The effect of alcohol intake on breastfeeding duration in Australian women. Acta Paediatrica, 97, 624629. doi:10.1111/j.1651-2227.2008.00760.x

Giles, M., Connor, S., McClenahan, C., \& Mallet, J. (2010). Attitudes to breastfeeding among adolescents. Journal of Human Nutrition and Dietetics, 23, 285-293. doi:10.1111/j.1365277X.2010.01048.X

Giles, M., McClenahan, C., Armour, C., Millar, S., Rae, G., Mallett, J., \& Stewart- Knox, B. (2014). Evaluation of a theory of planned behaviour-based breastfeeding intervention in Northern Irish Schools using a randomized cluster design. British Journal of Health Psychology, 19, 16-35. doi:10.1111/bjhp.12024

Gisore, P., Kaseje, D., Were, F., \& Ayuku, D. (2014). Motivational interviewing intervention on health- seeking behaviors of pregnant women in western Kenya. Journal of Applied Biobehavioral Research, 19, 144-156. doi:10.1111/jabr.12020

Glanz, K., Rimer, B.K. \& Lewis, F.M. (2002). Health behavior and health education: Theory, research and practice. San Francisco: Wiley \& Sons.

Göksen, F. (2002). Normative vs. attitudinal considerations in breastfeeding behavior: Multifaceted social influences in a developing country context. Social Science \& Medicine, 54, 1743-1753. doi:10.1016/S0277-9536(01)00145-9

Graffy, J., \& Taylor, J. (2005). What information, advice, and support do women want with breastfeeding? Birth, 32, 179-186. doi:10.1111/j.0730-7659.2005.00367.x

Graves, L. E., Turner, S., Nader, M., \& Sinha, S. (2016). Breastfeeding and opiate substitution therapy: Starting to understand infant feeding choices. Substance Abuse: Research and Treatment, 10, 43-47. doi:10.4137/SART.S34553 
Gregory, E. F., Gross, S. M., Nguyen, T. Q., Butz, A. M., \& Johnson, S. B. (2016). WIC participation and breastfeeding at 3 months postpartum. Maternal and Child Health Journal, 1-10. doi:10.1007/s10995-016-1977-1

Grieken, A., Velde, S., Vlasblom, J. D., Raat, H., Beltman, P., Hoir, M.. . Broeren, S. (2013). Primary prevention of overweight in preschool children, the BeeBOFT study (breastfeeding, breakfast daily, outsideplaying, few sweet drinks, less TV viewing): Design of a cluster randomized controlled trial. BMC Public Health, 13, 974-974. doi:10.1186/1471-2458-13-974

Guo, J. L., Wang, T. F., Liao, J. Y., \& Huang, C. M. (2016). Efficacy of the theory of planned behavior in predicting breastfeeding: meta-analysis and structural equation modeling. Applied Nursing Research, 29, 37-42. doi:10.1016/j.apnr.2015.03.016

Guo, Q., Johnson, C. A., Unger, J. B., Lee, L., Xie, B., Chou, C. P., ... \& Pentz, M. (2007). Utility of the theory of reasoned action and theory of planned behavior for predicting Chinese adolescent smoking. Addictive Behaviors, 32, 1066-1081. doi:10.1016/j.addbeh.2006.07.015

Handmaker, N., Miller, W., \& Manicke, M. (1999). Findings of a pilot study of motivational interviewing with pregnant drinkers. Journal of Studies on Alcohol, 60, 285-287. doi:10.15288/jsa.1999.60.285

Hannula, L., Kaunonen, M., \& Tarkka, M. (2008). A systematic review of professional support interventions for breastfeeding. Journal of Clinical Nursing, 17, 1132-1143. doi:10.1111/j.1365-2702.2007.02239.x

Hansen, M. N., Ezeonodo, A., Nyqvist, K. H., Haggkvist, A., Maastrup, R., Hannula, L.. . Högskolan i Skövde. (2013). Expansion of the baby-friendly hospital initiative ten steps 
to successful breastfeeding into neonatal intensive care: Expert group

recommendations. Journal of Human Lactation, 29, 300-309.

doi:10.1177/0890334413489775

Hanson, L. A. (2004). Immunobiology of human milk: How breastfeeding protects babies. Amarillo, TX: Pharmasoft Publishing.

Harden, S. M., Gaglio, B., Shoup, J. A., Kinney, K. A., Johnson, S. B., Brito, F., ... Estabrooks, P. A. (2015). Fidelity to and comparative results across behavioral interventions evaluated through the RE-AIM framework: A systematic review. Systematic Reviews, 4, 155. doi:10.1186/s13643-015-0141-0

Haroon, S., Das, J., Salam, R., Imdad, A., \& Bhutta, Z. (2013). Breastfeeding promotion interventions and breastfeeding practices: A systematic review. BMC Public Health, 13(Suppl 3), S20. doi:10.1186/1471-2458-13-S3-S20.

Hatton, D. C., Harrison-Hohner, J., Coste, S., Dorato, V., Curet, L. B., \& McCarron, D. A. (2005). Symptoms of postpartum depression and breastfeeding. Journal of Human Lactation, 21, 444-449. doi:10.1177/0890334405280947

Hauck, Y., Graham-Smith, C., McInerney, J., \& Kay, S. (2011). Western Australian women's perceptions of conflicting advice around breast feeding. Midwifery, 27(5), E156-E162. doi:10.1016/j.midw.2010.02.003

Hausenblas, H. A., \& Symons Downs, D. (2004). Prospective examination of the Theory of Planned Behavior applied to exercise behavior during women's first trimester of pregnancy. Journal of Reproductive and Infant Psychology, 22, 199-210. doi:10.1080/02646830410001723788 
Hayes, C., Collins, C., O'Carroll, H., Wyse, E., Gunning, M., Geary, M., \& Kelleher, C. (2013). Effectiveness of motivational interviewing in influencing smoking cessation in pregnant and postpartum disadvantaged women. Nicotine \& Tobacco Research, 15, 969-977. doi:10.1093/ntr/nts225

Health behavior. (n.d.) Mosby's Medical Dictionary, 8th edition. (2009). Retrieved from http://medical-dictionary.thefreedictionary.com/health+behavior

Heaman, M. I., Sword, W., Elliott, L., Moffatt, M., Helewa, M. E., Morris, H., ... \& Cook, C. (2015). Barriers and facilitators related to use of prenatal care by inner-city women: Perceptions of health care providers. BMC Pregnancy and Childbirth, 15, 2. doi:10.1186/s12884-015-0431-5

Heck, K. E., Schoendorf, K. C., Chavez, G. F., \& Braveman, P. (2003). Does postpartum length of stay affect breastfeeding duration? A population-based study. Birth, 30, 153-159. doi:10.1046/j.1523-536X.2003.00239.x

Hettema, J. E., \& Hendricks, P. S. (2010). Motivational interviewing for smoking cessation: A meta-analytic review. Journal of Consulting and Clinical Psychology, 78, 868-884. doi: $10.1037 / \mathrm{a} 0021498$

Hettema, J., Steele, J., \& Miller, W. (2005). Motivational interviewing. Annual Review of Clinical Psychology, 1, 91-111. doi:10.1146/annurev.clinpsy.1.102803.143833

Heymann, J., Raub, A., \& Earle, A. (2013). Breastfeeding policy: A globally comparative analysis. Bulletin of the World Health Organization, 91, 398-406. doi:

10.2471/BLT.12.109363 
Hibbard, J. H., Mahoney, E. R., Stock, R., \& Tusler, M. (2007). Do increases in patient activation result in improved self-management behaviors? Health Services Research, 42, 1443-1463. doi:10.1111/j.1475-6773.2006.00669.x

Hogan, M., Westcott, C., \& Griffiths, M. (2005). Randomized, controlled trial of division of tongue- tie in infants with feeding problems. Journal of Paediatrics and Child Health, 41, 246-250. doi:10.1111/j.1440-1754.2005.00604.x

Holbrook, K. E., White, M. C., Heyman, M. B., \& Wojcicki, J. M. (2013). Maternal sociodemographic characteristics and the use of the Iowa Infant Attitude Feeding Scale to describe breastfeeding initiation and duration in a population of urban, Latina mothers: A prospective cohort study. International Breastfeeding Journal, 8, 7. doi:10.1186/17464358-8-7

Holick, M. F., Binkley, N. C., Bischoff-Ferrari, H. A., Gordon, C. M., Hanley, D. A., Heaney, R. P.,...Weaver, C. M. (2011). Evaluation, treatment, and prevention of vitamin D deficiency: An Endocrine Society clinical practice guideline. Journal of Clinical Endocrinology \& Metabolism, 96, 1911-1930. doi:10.1210/jc.2011-0385.

Howe- Heyman, A., \& Lutenbacher, M. (2016). The Baby- Friendly hospital initiative as an intervention to improve breastfeeding rates: A review of the literature. Journal of Midwifery \& Women's Health, 61(1), 77-102. doi:10.1111/jmwh.12376

Huang, K. Y., Caughy, M. O. B., Genevro, J. L., \& Miller, T. L. (2005). Maternal knowledge of child development and quality of parenting among White, African-American and Hispanic mothers. Journal of Applied Developmental Psychology, 26, 149-170. doi:10.1016/j.appdev.2004.12.001 
Huang, R., \& Yang, M. (2015). Paid maternity leave and breastfeeding practice before and after California's implementation of the nation's first paid family leave program. Economics \& Human Biology, 16, 45-59. doi:10.1016/j.ehb.2013.12.009

Hunsley, J. (1992). Development of the treatment acceptability questionnaire. Journal of Psychopathology and Behavioral Assessment, 14, 55-64.

Huynh, H., \& Feldt, L. S. (1976). Estimation of the Box correction for degrees of freedom from sample data in randomized block and split-plot designs. Journal of Educational Statistics, 1, 69-82. doi:10.3102/10769986001001069

IBM Corp. (2012). IBM SPSS Statistics for Windows, Version 21.0. Armonk, NY: IBM Corp. Ip, S., Chung, M., Raman, G., Trikalinos, T., \& Lau, J. (2009). A summary of the agency for healthcare research and quality's evidence report on breastfeeding in developed countries. Breastfeeding Medicine, 4, S17-S30. doi:10.1089/bfm.2009.0050

Ismail, A. I., Ondersma, S., Willem Jedele, J. M., Little, R. J., \& Lepkowski, J. M. (2011). Evaluation of a brief tailored motivational intervention to prevent early childhood caries. Community Dentistry and Oral Epidemiology, 39, 433-448. doi:10.1111/j.16000528.2011.00613.x

Jackson, D. B. (2016). The association between breastfeeding duration and attachment: A genetically informed analysis. Breastfeeding Medicine, 11, 297-304. doi:10.1089/bfm.2016.0036

Jackson, K. T., and Dennis, C.-L. (2017) Lanolin for the treatment of nipple pain in breastfeeding women: a randomized controlled trial. Maternal \& Child Nutrition, 13, e12357. doi:10.1111/mcn.12357 
Jansen, J., Weerth, C. D., \& Riksen-Walraven, J. M. (2008). Breastfeeding and the mother-infant relationship_A review. Developmental Review, 28, 503-521. doi:10.1016/j.dr.2008.07.001

Jansson, L. M., Spencer, N., McConnell, K., Velez, M., Tuten, M., Harrow, C. A., ... \& Huestis, M. A. (2016). Maternal buprenorphine maintenance and lactation. Journal of Human Lactation, 32, 675-681. doi:10.1177/0890334416663198

Jensen, E. (2012). Participation in the supplemental nutrition program for women, infants and children (WIC) and breastfeeding: National, regional, and state level analyses. Maternal and Child Health Journal, 16, 624-631. doi:10.1007/s10995-011-0796-7

Jessri, M., Farmer, A. P., Maximova, K., Willows, N. D., \& Bell, R. C. (2013). Predictors of exclusive breastfeeding: Observations from the Alberta pregnancy outcomes and nutrition (APrON) study. BMC Pediatrics, 13, 77. doi:10.1186/1471-2431-13-77

Jones, J., Kogan, M., Singh, G., Dee, D., \& Grummer-Strawn, L. (2011). Factors associated with exclusive breastfeeding in the United States. Pediatrics, 128, 1117-1125. doi:10.1542/peds.2011-0841

Jones, K. M., Power, M. L., Queenan, J. T., \& Schulkin, J. (2015). Racial and ethnic disparities in breastfeeding. Breastfeeding Medicine, 10, 186-196. doi:10.1089/bfm.2014.0152

Jung, C. (2015). Lactivism: How feminists and fundamentalists, hippies and yuppies, and physicians and politicians made breastfeeding big business and bad policy. New York, NY: Basic Books.

Karatay, G., Kublay, G., \& Emiroğlu, O. N. (2010). Effect of motivational interviewing on smoking cessation in pregnant women. Journal of Advanced Nursing, 66, 1328-1337. doi:10.1111/j.1365-2648.2010.05267.x 
Kaunonen, M., Hannula, L., \& Tarkka, M. (2012). A systematic review of peer support interventions for breastfeeding. Journal of Clinical Nursing, 21, 1943-1954. doi:10.1111/j.1365-2702.2012.04071.x

Kavanagh, K. F., Lou, Z., Nicklas, J. C., Habibi, M. F., \& Murphy, L. T. (2012). Breastfeeding knowledge, attitudes, prior exposure, and intent among undergraduate students. Journal of Human Lactation, 28, 556-564. doi:10.1177/0890334412446798

Kazdin, A. E. (2003). Research design in clinical psychology (4th ed.). Boston, MA: Allyn and Bacon.

Khan, J., Vesel, L., Bahl, R., \& Martines, J. C. (2015). Timing of breastfeeding initiation and exclusivity of breastfeeding during the first month of life: Effects on neonatal mortality and morbidity — a systematic review and meta-analysis. Maternal and Child Health Journal, 19, 468-479. doi:10.1007/s10995-014-1526-8

Kim, J. H., Fiese, B. H., \& Donovan, S. M. (2017). Breastfeeding is natural but not the cultural norm: a mixed-methods study of first-time breastfeeding, African American mothers participating in WIC. Journal of Nutrition Education and Behavior, 49, S151-S161. doi:10.1016/j.jneb.2017.05.342

Kinser, P. A., \& Robins, J. L. (2013). Control group design: Enhancing rigor in research of mind-body therapies for depression. Evidence-Based Complementary and Alternative Medicine, 2013, 140467. doi:10.1155/2013/140467.

Kloeblen-Tarver, A. S., Thompson, N. J., \& Miner, K. R. (2002). Intent to breast-feed: The impact of attitudes, norms, parity, and experience. American Journal of Health Behavior, 26, 182-187. doi:10.5993/AJHB.26.3.3 
Kogan, M. D., Singh, G. K., Dee, D. L., Belanoff, C., \& Grummer-Strawn, L. M. (2008).

Multivariate analysis of state variation in breastfeeding rates in the United States. American Journal of Public Health, 98, 1872-1880. doi:10.2105/AJPH.2007.127118.

Kramer, M. S., Aboud, F., Mironova, E., Vanilovich, I., Platt, R. W., Matush, L., ... \& Collet, J. P. (2008). Breastfeeding and child cognitive development: New evidence from a large randomized trial. Archives of General Psychiatry, 65, 578-584. doi:10.1001/archpsyc.65.5.578.

Kramer, M. S., Chalmers, B., Hodnett, E. D., Sevkovskaya, Z., Dzikovich, I., Shapiro, S., et al. (2001). Promotion of breastfeeding intervention trial [PROBIT]: A randomized trial in the Republic of Belarus. JAMA, 285, 413-420. doi:10.1001/jama.285.4.413

Krosnick, J. A., \& Alwin, D. F. (1989). Aging and susceptibility to attitude change. Journal of Personality and Social Psychology, 57, 416-425.

Labarere, J., Stone, R. A., Obrosky, D. S., Yealy, D. M., Meehan, T. P., Auble, T. E.. . Fine, M. J. (2006). Factors associated with the hospitalization of low-risk patients with community-acquired pneumonia in a cluster-randomized trial. Journal of General Internal Medicine, 21, 745-752. doi:10.1111/j.1525-1497.2006.00510.x

Labbok, M. H., \& Starling, A. (2012). Definitions of breastfeeding: Call for the development and use of consistent definitions in research and peer-reviewed literature. Breastfeeding Medicine, 7, 397-402. doi:10.1089/bfm.2012.9975

Labbok, M., \& Krasovec, K. (1990). Toward consistency in breastfeeding definitions. Studies in Family Planning, 226-230. doi:10.2307/1966617 
LaBrie, J. W., Quinlan, T., Schiffman, J. E., \& Earleywine, M. E. (2005). Performance of alcohol and safer sex change rulers compared with readiness to change questionnaires. Psychology of Addictive Behaviors, 19, 112-115. doi:10.1037/0893-164X.19.1.112

Lai, D. T., Cahill, K., Qin, Y., \& Tang, J. L. (2010). Motivational interviewing for smoking cessation. Cochrane Database Systematic Reviews, 3, 1-37. doi:10.1002/14651858.CD006936.pub2

Langellier, B. A., Chaparro, M. P., Wang, M. C., Koleilat, M., \& Whaley, S. E. (2014). The new food package and breastfeeding outcomes among women, infants, and children participants in Los Angeles County. American Journal of Public Health, 104, S112-S118. doi:10.2105/AJPH.2013.301330

Lawton, R., Ashley, L., Dawson, S., Waiblinger, D., \& Conner, M. (2012). Employing an extended Theory of Planned Behaviour to predict breastfeeding intention, initiation, and maintenance in White British and South- Asian mothers living in Bradford. British Journal of Health Psychology, 17, 854-871. doi:10.1111/j.2044-8287.2012.02083.x

Lee, C. Y. W., \& Koren, G. (2010). Maternal obesity: Effects on pregnancy and the role of preconception counselling. Journal of Obstetrics and Gynaecology, 30, 101-106. doi:10.3109/01443610903474355

Levison, J., Weber, S., \& Cohan, D. (2014). Breastfeeding and HIV-infected women in the United States: harm reduction counseling strategies. Clinical Infectious Diseases, 59, 304-309. doi:10.1093/cid/ciu272

Lind, J. N., Perrine, C. G., Li, R., Scanlon, K. S., Grummer-Strawn, L. M., \& CDC. (2014). Racial disparities in access to maternity care practices that support breastfeeding-United States, 2011. MMWR Morbidity and Mortality Weekly Report, 63, 725-728. 
Lindson-Hawley N., Thompson T. P., Begh, R. (2015). Motivational interviewing for smoking cessation. Cochrane Database of Systematic Reviews, 3, 1-78. doi:10.1002/14651858.CD006936.pub3.

Lumbiganon, P., Martis, R., Laopaiboon, M., Festin, M. R., Ho, J. J., \& Hakimi, M. (2012). Antenatal breastfeeding education for increasing breastfeeding duration. The Cochrane Database of Systematic Reviews, 9, CD006425. doi:10.1002/14651858.CD006425.pub3

Lund, H. G., Turkle, J., Jewell, M. L., \& Murphy, D. K. (2016). Low risk of skin and nipple sensitivity and lactation issues after primary breast augmentation with form-stable silicone implants: Follow-up in 4927 subjects. Aesthetic Surgery Journal, 36, 672-680. doi:10.1093/asj/sjv266)

Lundahl, B., \& Burke, B. L. (2009). The effectiveness and applicability of motivational interviewing: A practice- friendly review of four meta- analyses. Journal of Clinical Psychology, 65, 1232-1245. doi:10.1002/jclp.20638

MacPhee, D. (1981). Knowledge of Infant Development Inventory. Princeton, NJ: Educational Testing Service.

Madson, M. B., Loignon, A. C., \& Lane, C. (2009). Training in Motivational Interviewing: A systematic review. Journal of Substance Abuse Treatment, 36, 101-109. doi:10.1016/j.jsat.2008.05.005

Martins, R. K. (2008). Implications of Motivational Interviewing and oral hygiene instruction for the reduction of oral health disparities among pregnant women. (Unpublished doctoral dissertation). West Virginia University, Morgantown, WV. 
Martins, R. K., \& McNeil, D. W. (2009). Review of motivational interviewing in promoting health behaviors. Clinical Psychology Review, 29, 283-293. doi:10.1016/j.cpr.2009.02.001

Mathews, M. E., Leerkes, E. M., Lovelady, C. A., \& Labban, J. D. (2014). Psychosocial predictors of primiparous breastfeeding initiation and duration. Journal of Human Lactation, 30, 480-487. doi:10.1177/0890334414537707

Matthey, S., \& Ross-Hamid, C. (2011). The validity of DSM symptoms for depression and anxiety disorders during pregnancy. Journal of Affective Disorders, 133, 546-552. doi:10.1016/j.jad.2011.05.004

Mayo Foundation for Medical Education and Research. (2014, July 11). Fetal development: The third trimester. Retrieved from http://www.mayoclinic.org/healthylifestyle/pregnancy-week-by-week/in-depth/fetal-development/art-20045997

McCambridge, J., \& Strang, J. (2004). The efficacy of single-session motivational interviewing in reducing drug consumption and perceptions of drug-related risk and harm among young people: results from a multi-site cluster randomized trial. Addiction, 99, 39-52. doi:10.1111/j.1360-0443.2004.00564.X

McNeil, D. W., Addicks, S. H., \& Randall, C. L. (in press). Motivational Interviewing and motivational interactions for health behavior change and maintenance. Oxford handbook of online only_Psychology. doi:10.1093/oxfordhb/9780199935291.013.21

McNeil, D. W., Crout, R. J., Marazita, M. L. (2012). Oral health in Appalachia. In: Ludke R. L., Obermiller P. J., editors. Appalachian Health and Well-Being (pp. 275-294). Lexington, KY, USA: University Press of Kentucky; 2012. pp. 275-294. 
McNiel, M. E., Labbok, M. H., \& Abrahams, S. W. (2010). What are the risks associated with formula feeding? A re-analysis and review. Birth, 37, 50-58. doi:10.1111/j.1523536X.2009.00378.x

Meedya, S., Fahy, K., \& Kable, A. (2010). Factors that positively influence breastfeeding duration to 6 months: a literature review. Women and Birth, 23, 135-145. doi:10.1016/j.wombi.2010.02.002

Miller, W. R., \& Rollnick, S. (2009). Ten things that motivational interviewing is not. Behavioural and Cognitive Psychotherapy, 37, 129-140. doi:10.1017/S1352465809005128

Miller, W. R., \& Rollnick, S. (2013). Motivational interviewing: Helping people change (3rd ed.). New York, NY: Guilford Press.

Miller, W. R., \& Rose, G. S. (2009). Motivational interviewing in relational context. American Psychologist, 65, 298-299. doi:10.1037/a0019487

Montgomery-Reagan, K., Bianco, J. A., Heh, V., Rettos, J., \& Huston, R. S. (2009). Prevalence and correlates of high body mass index in rural Appalachian children aged 6-11 years. Rural and Remote Health, 9, 1234.

Mora, A., Russell, D. W., Dungy, C. I., Losch, M., \& Dusdieker, L. (1999). The Iowa infant feeding attitude scale: Analysis of reliability and validity. Journal of Applied Social Psychology, 29, 2362-2380. doi:10.1111/j.1559-1816.1999.tb00115.x

Moral, R. R., de Torres, L. A. P., Ortega, L. P., Larumbe, M. C., Villalobos, A. R., García, J. A. F., ... \& Study, C. G. A. A. (2015). Effectiveness of motivational interviewing to improve therapeutic adherence in patients over 65 years old with chronic diseases: A cluster 
randomized clinical trial in primary care. Patient Education and Counseling, 98, 977983. doi:10.1016/j.pec.2015.03.008

Moretti, M. E., Lee, A., \& Ito, S. (2000). Which drugs are contraindicated during breastfeeding? Practice guidelines. Canadian Family Physician, 46, 1753-1757.

Morland- Schultz, K., \& Hill, P. D. (2005). Prevention of and therapies for nipple pain: A systematic review. Journal of Obstetric, Gynecologic, \& Neonatal Nursing, 34, 428437. doi: $10.1177 / 0884217505276056$

Moyers, T. B., Manuel, J. K., \& Ernst, D. (2014). Motivational Interviewing Treatment Integrity Coding Manual 4.1. Unpublished manual. Retrieved from http://casaa.unm.edu/download/MITI4_1.pdf

Moyers, T. B., Rowell, L. N., Manuel, J. K., Ernst, D., \& Houck, J. M. (2016). The Motivational Interviewing Treatment Integrity Code (MITI 4): Rationale, preliminary reliability and validity. Journal of Substance Abuse Treatment. doi:10.1016/j.jsat.2016.01.001

Mozingo, J. N., Davis, M. W., Droppleman, P. G., \& Merideth, A. (2000). "It wasn't working": Women's experiences with short-term breastfeeding. American Journal of Maternal/Child Nursing, 25, 120-126.

Mulimba, A. A. C., \& Byron-Daniel, J. (2014). Motivational interviewing-based interventions and diabetes mellitus. British Journal of Nursing, 23, 8-14. doi:10.12968/bjon.2014.23.1.8

Nagy, E., Orvos, H., Pal, A., Kovacs, L., \& Loveland, K. (2001). Breastfeeding duration and previous breastfeeding experience. Acta Paediatrica, 90, 51-56.

Naidu, R., Nunn, J., \& Irwin, J. (2015). The effect of motivational interviewing on oral healthcare knowledge, attitudes and behaviour of parents and caregivers of preschool 
children: An exploratory cluster randomised controlled study. BMC Oral Health, 15, 101. doi:10.1186/s12903-015-0068-9

Napoli, A. D., Lallo, D. D., Fortes, C., Franceschelli, C., Armeni, E., \& Guasticchi, G. (2004). Home breastfeeding support by health professionals: Findings of a randomized controlled trial in a population of Italian women. Acta Paediatrica, 93, 1108-1114. doi:10.1080/08035250410028525

National Center for Infants, Toddlers and Families. (2014). Zero to Three. Washington, DC: Author.

National Conference of State Legislatures. (2017). Breastfeeding state laws. Retrieved from http://www.ncsl.org/research/health/breastfeeding-state-laws.aspx

Neiswanger, K., McNeil, D. W., Foxman, B., Govil, M., Cooper, M. E., ...\& Marazita, M. L. (2015). Oral health in a sample of pregnant women from Northern Appalachia (20112015). International Journal of Dentistry, 2015, 469376. doi:10.1155/2015/469376.

Nelson, A. M. (2007). Maternal- newborn nurses' experiences of inconsistent professional breastfeeding support. Journal of Advanced Nursing, 60, 29-38. doi:10.1111/j.13652648t.2007.04373.x

Newhook, J., T., Newhook, L. A., Midodzi, W. K., Murphy Goodridge, J., Burrage, L., Gill, N., ... \& Twells, L. (2017). Poverty and breastfeeding: Comparing determinants of early breastfeeding cessation incidence in socioeconomically marginalized and privileged populations in the FiNaL Study. Health Equity, 1, 96-102. doi:10.1089/heq.2016.0028.

Newton, F. (2014). "The new era of communication among Americans." GallupNews. 
Newton, K., Chaudhuri, J., Grossman, X., \& Merewood, A. (2009). Factors associated with exclusive breastfeeding among Latina women giving birth at an inner-city baby-friendly hospital. Journal of Human Lactation, 25, 28-33. doi:10.1177/0890334408329437

Nickel, N. C., Labbok, M. H., Hudgens, M. G., \& Daniels, J. L. (2013). The extent that noncompliance with the ten steps to successful breastfeeding influences breastfeeding duration. Journal of Human Lactation, 29, 59-70. doi:10.1177/0890334412464695

Noel- Weiss, J., Rupp, A., Cragg, B., Bassett, V., \& Woodend, A. K. (2006). Randomized controlled trial to determine effects of prenatal breastfeeding workshop on maternal breastfeeding self-efficacy and breastfeeding duration. Journal of Obstetric, Gynecologic, \& Neonatal Nursing, 35, 616-624. doi:10.1111/j.1552-6909.2006.00077.x

Nunnally, J. C., \& Bernstein, I. (1994). Psychometric theory. 3rd ed. New York, NY: McGrawHill.

O’Brien, M., Buikstra, E., Fallon, T., \& Hegney, D. (2009). Exploring the influence of psychological factors on breastfeeding duration, phase 1: Perceptions of mothers and clinicians. Journal of Human Lactation, 25, 55-63. doi:10.1177/0890334408326071

Odom, E. C., Li, R., Scanlon, K. S., Perrine, C. G., \& Grummer-Strawn, L. (2013). Reasons for earlier than desired cessation of breastfeeding. Pediatrics, e726-e732. doi:10.1542/peds.2012-1295

Olsen, Y., \& Sharfstein, J. M. (2014). Confronting the stigma of opioid use disorder-and its treatment. JAMA, 311, 1393-1394. doi:10.1001/jama.2014.2147.

Osterman, R. L., Carle, A. C., Ammerman, R. T., \& Gates, D. (2014). Single-session motivational intervention to decrease alcohol use during pregnancy. Journal of Substance Abuse Treatment, 47, 10-19. doi:10.1016/j.jsat.2014.01.009 
Osterman, R., \& Dyehouse, J. Effects of a motivational interviewing intervention to decrease prenatal alcohol use. (2012). Western Journal of Nursing Research, 34, 434-454. doi:10.1177/0193945911402523

Parry, K. C., Tully, K. P., Moss, S. L., \& Sullivan, C. S. (2017). Innovative prenatal breastfeeding education curriculum: Ready, Set, BABY. Journal of Nutrition Education and Behavior, 49, S214-S216. doi:10.1016/j.jneb.2017.05.348

Patient Protection and Affordable Care Act, 42 U.S.C. $§ 18001$ et seq. (2010).

Peres, K. G., Cascaes, A. M., Nascimento, G. G., \& Victora, C. G. (2015). Effect of breastfeeding on malocclusions: A systematic review and meta- analysis. Acta Paediatrica, 104(S467), 54-61. doi:10.1111/apa.13103

Pérez-Escamilla, R., \& Sellen, D. (2015). Equity in breastfeeding: Where do we go from here? Journal of Human Lactation, 31, 12-14. doi:10.1177/0890334414561062

Phillips, R. M., Goldstein, M., Hougland, K., Nandyal, R., Pizzica, A., Santa-Donato, A., ... \& Yost, E. (2013). Multidisciplinary guidelines for the care of late preterm infants. Journal of Perinatology, 33, S5-S22. doi:10.1038/jp.2013.53

Polk, D. E., Kim, S., Manz, M., \& Weyant, R. J. (2015). Is there an Appalachian disparity in dental caries in Pennsylvania schoolchildren? Community Dentistry and Oral Epidemiology, 43, 24-32. doi:10.1111/cdoe.12135

Quinn, G. P., Detman, L. A., Bell-Ellison, B. A. (2008). Missed appointments in perinatal care: Response variations in quantitative versus qualitative instruments. The Journal of Medical Practice Management: MPM, 23, 307-313.

Rasouli, M., Atash Sokhan, G., Keramat, A., Khosravi, A., Fooladi, E., \& Mousavi, S. A. (2017). The impact of motivational interviewing on participation in childbirth preparation 
classes and having a natural delivery: a randomised trial. BJOG: An International Journal of Obstetrics \& Gynaecology, 124, 631-639. doi:10.1111/1471-0528.14397

Redshaw, M., Malouf, R., Gao, H., \& Gray, R. (2013). Women with disability: The experience of maternity care during pregnancy, labour and birth and the postnatal period. BMC Pregnancy and Childbirth, 13, 174. doi:10.1186/1471-2393-13-174

Reeve, J. R., Gull, S. E., Johnson, M. H., Hunter, S., \& Streather, M. (2004). A preliminary study on the use of experiential learning to support women's choices about infant feeding. European Journal of Obstetrics \& Gynecology and Reproductive Biology, 113, 199-203. doi:10.1016/j.ejogrb.2003.07.002

Resnicow, K., Jackson, A., Wang, T., De, A. K., McCarty, F., Dudley, W. N., \& Baranowski, T. (2001). A motivational interviewing intervention to increase fruit and vegetable intake through black churches: Results of the eat for life trial. American Journal of Public Health, 91, 1686-1693. doi:10.2105/AJPH.91.10.1686

Retrieved from http://news.gallup.com/poll/179288/new-era-communicationamericans.aspx

Reynolds, D., Hennessy, E., \& Polek, E. (2014). Is breastfeeding in infancy predictive of child mental well- being and protective against obesity at 9 years of age? Child: Care, Health and Development, 40, 882-890. doi:10.1111/cch.12126

Riordan, J., \& Wambach, K. (2010). Breastfeeding and human lactation. Sudbury, MA: Jones \& Bartlett Learning.

Robiquet, P., Zamiara, P., Rakza, T., Deruelle, P., Mestdagh, B., Blondel, G.. . Subtil, D. (2016). Observation of skin-to-skin contact and analysis of factors linked to failure to breastfeed 
within 2 hours after birth. Breastfeeding Medicine, 11, 126-132.

doi:10.1089/bfm.2015.0160.

Rojjanasrirat, W., Nelson, E. L., \& Wambach, K. A. (2012). A pilot study of home-based videoconferencing for breastfeeding support. Journal of Human Lactation, 28, 464-467. doi: $10.1177 / 0890334412449071$

Rosen, I. M., Krueger, M. V., Carney, L. M., \& Graham, J. A. (2008). Prenatal breastfeeding education and breastfeeding outcomes. American Journal of Maternal/Child Nursing, 33, 315-319. doi:10.1097/01.NMC.0000334900.22215.ec

Rosenberger, W. F. and Lachin, J. M. (2016) Covariate-Adaptive Randomization, in Randomization in Clinical Trials: Theory and Practice ( $2^{\text {nd }}$ ed., pp. 169-188). Hoboken, NJ: John Wiley \& Sons, Inc.

Rowe-Murray, H. J., \& Fisher, J. R. (2002). Baby friendly hospital practices: Cesarean section is a persistent barrier to early initiation of breastfeeding. Birth, 29, 124-131. doi:10.1046/j.1523-536X.2002.00172.x

Rubak, S., Sandbæk, A., Lauritzen, T., \& Christensen, B. (2005). Motivational interviewing: A systematic review and meta-analysis. British Journal of General Practice, 55, 305-312.

Russ, K. A. (2010). Working with clients of Appalachian culture. VISTAS Online. Retrieved from http://counselingoutfitters.com/vistas/vistas10/Article_69.pdf

Rutledge, S. E. (2007). Single-session motivational enhancement counseling to support change toward reduction of HIV transmission by HIV positive persons. Archives of Sexual Behavior, 3, 313-319. doi:10.1007/s10508-006-9077-8 
Ryan, A. S., \& Zhou, W. (2006). Lower breastfeeding rates persist among the Special Supplemental Nutrition Program for Women, Infants, and Children participants, 19782003. Pediatrics, 117, 1136-1146. doi:10.1542/peds.2005-1555

Ryser, F. G. (2004). Breastfeeding attitudes, intention, and initiation in low-income women: The effect of the best start program. Journal of Human Lactation, 20, 300-305. doi:10.1177/0890334404266985

Sakalidis, V. S., Williams, T. M., Hepworth, A. R., Garbin, C. P., Hartmann, P. E., Paech, M. J...Geddes, D. T. (2013). A comparison of early sucking dynamics during breastfeeding after cesarean section and vaginal birth. Breastfeeding Medicine, 8, 79-85. doi:10.1089/bfm.2012.0018

Saltzman, J., Cole, N., Bost, K. K., Fiese, B. H., \& Donovan, S. (2017). Responsive feeding and breastfeeding moderate the association between maternal attachment security and overeating in 18-month old infants in the STRONG Kids 2 cohort. The FASEB Journal, 31, 169-174. doi:10.1096/fj.1530-6860

Satcher, D., \& Higginbotham, E. J. (2008). The public health approach to eliminating disparities in health. American Journal of Public Health, 98, 400-403. doi:10.2105/AJPH.2007.123919

Schilling, R. F., El-Bassel, N., Finch, J. B., Roman, R. J., \& Hanson, M. (2002). Motivational interviewing to encourage self-help participation following alcohol detoxification. Research on Social Work Practice, 12, 711-730.

doi:10.1177/104973102237469 
Schmied, V., Beake, S., Sheehan, A., McCourt, C., \& Dykes, F. (2011). Women's perceptions and experiences of breastfeeding support: A metasynthesis. Birth, 38, 49-60. doi:10.1111/j.1523-536X.2010.00446.x

Schwarz, E. B., Ray, R. M., Stuebe, A. M., Allison, M. A., Ness, R. B., Freiberg, M. S., \& Caulety, J. A. (2009). Duration of lactation and risk factors for maternal cardiovascular disease. Obstetrics and Gynecology, 113, 974-982.

Scott, J. A., Binns, C. W., Oddy, W. H., \& Graham, K. I. (2006). Predictors of breastfeeding duration: Evidence from a cohort study. Pediatrics, 117, e646-e655. doi:10.1542/peds.2005-1991

Scott, J. A., Shaker, I., \& Reid, M. (2004). Parental attitudes towards breastfeeding: Their association with feeding outcome at hospital discharge. Birth, 31, 125-131. doi:10.1111/j.0730-7659.2004.00290.x.

Shah, P. S. (2010). Parity and low birth weight and preterm birth: a systematic review and metaanalyses. Acta Obstetricia et Gynecologica Scandinavica, 89, 862-875. doi:10.3109/00016349.2010.486827

Shanyinde, M., Pickering, R. M., \& Weatherall, M. (2011). Questions asked and answered in pilot and feasibility randomized controlled trials. BMC Medical Research Methodology, 11, 117. doi:10.1186/1471-2288-11-117

Shaw, G. M. (2011). Having children after cancer: How to make informed choices before and after treatment and build the family of your dreams. Berkeley, CA: Crown Publishing Group.

Sheard, N. (1988). The role of breast milk in the development of the gastrointestinal tract. Nutrition Reviews. 48, 1-8 
Sittlington, J., Stewart-Knox, B., Wright, M., Bradbury, I., \& Scott, J. A. (2007). Infant-feeding attitudes of expectant mothers in Northern Ireland. Health Education Research, 22, 561570. doi:10.1093/her/cyl113

Snyder, A., \& Thatcher, E. (2014). From the trunk of a volkswagen beetle: A mobile nursing clinic in Appalachia. Family \& Community Health, 37, 239-247. doi:10.1097/FCH.0000000000000028

Somerville, S., Dedman, K., Hagan, R., Oxnam, E., Wettinger, M., Byrne, S.,...\& Page, A. C. (2014). The Perinatal Anxiety Screening Scale: Development and preliminary validation. Archives of Women's Mental Health 17, 443-454. doi:10.1007/s00737-0140425-8

Sparks, P. J. (2010). Rural-urban differences in breastfeeding initiation in the United States. Journal of Human Lactation, 26, 118-129. doi:10.1177/0890334409352854

Special Supplemental Nutrition Program for Women, Infants and Children (WIC): Revisions in the WIC Food Packages; Final Rule, 79 Fed. Reg. 12274 (March 4, 2014). (To be codified at 7 C. F. R. pt. 246.

Stearns, C. A. (1999). Breastfeeding and the good maternal body. Gender \& Society, 13, 308325. doi:10.1177/089124399013003003

Steinberg, M. (2011). Clinical perspectives on motivational interviewing in diabetes care. Diabetes Spectrum, 24, 179-181. doi:10.2337/diaspect.24.3.179

Steinberg, M., \& Miller, W. R. (2015). Motivational Interviewing in diabetes care. New York, NY: Guilford.

Stuebe, A. (2009). The risks of not breastfeeding for mothers and infants. Reviews in Obstetrics and Gynecology, 2, 222-231. doi:10.3909/riog0093 
Stuebe, A. M., \& Schwarz, E. B. (2010). The risks and benefits of infant feeding practices for women and their children. Journal of Perinatology, 30, 155-162. doi:10.1038/jp.2009.107

Stuebe, A. M., Michels, K. B., Willett, W. C., Manson, J. E., Rexrode, K., \& Rich-Edwards, J. W. (2009). Duration of lactation and incidence of myocardial infarction in middle to late adulthood. American Journal of Obstetrics and Gynecology, 200, 138e1-138e8. doi:10.1016/j.ajog.2008.10.001

Stuebe, A. M., Schwarz, E. B., Grewen, K., Rich-Edwards, J. W., Michels, K. B., Foster, E. M., Curhan, G., \& Forman, J. (2011). Duration of lactation and incidence of maternal hypertension: A longitudinal cohort study. American Journal of Epidemiology, 174, 1147-1158. doi:10.1093/aje/kwr227.

Substance Abuse and Mental Health Services Administration. (2016). Medication-assisted treatment. Retrieved from https://www.samhsa.gov/medication-assisted-treatment

Suresh, K. (2011). An overview of randomization techniques: an unbiased assessment of outcome in clinical research. Journal of Human Reproductive Sciences, 4, 8-11. doi:10.4103/0974-1208.82352

Tabachnick, B. G., \& Fidell, L. S. (2013). Using multivariate statistics. (6 ${ }^{\text {th }}$ Ed.). New York, NY: Pearson.

Tarrant, M., \& Dodgson, J. E. (2007). Knowledge, attitudes, exposure, and future intentions of Hong Kong university students toward infant feeding. Journal of Obstetric, Gynecologic, \& Neonatal Nursing, 36, 243-254. doi:10.1111/j.1552-6909.2007.00144.x 
Taveras, E. M., Capra, A. M., Braveman, P. A., Jensvold, N. G., Escobar, G. J., \& Lieu, T. A. (2003). Clinician support and psychosocial risk factors associated with breastfeeding discontinuation. Pediatrics, 112, 108-115. doi:10.1542/peds.112.1.108

Thomas-Jackson, S. C., Bentley, G. E., Keyton, K., Reifman, A., Boylan, M., \& Hart, S. L. (2016). In-hospital breastfeeding and intention to return to work influence mothers' breastfeeding intentions. Journal of Human Lactation, 32, 76-83.

doi:10.1177/0890334415597636

Thompson, D. R., Chair, S. Y., Chan, S. W., Astin, F., Davidson, P. M., \& Ski, C. F. (2011).

Motivational interviewing: A useful approach to improving cardiovascular health? Journal of Clinical Nursing, 20, 1236-1244. doi:10.1111/j.13652702.2010.03558.x

Thomson, G., Ebisch- Burton, K., \& Flacking, R. (2015). Shame if you do-shame if you don't: Women's experiences of infant feeding. Maternal \& Child Nutrition, 11, 33-46. doi:10.1111/mcn. 12148

Tsai, L. C., \& Doan, T. J. (2016). Breastfeeding among mothers on opioid maintenance treatment: A literature review. Journal of Human Lactation, 32, 521-529. doi:10.1177/0890334416641909

Tuthill, E. L., Butler, L. M., Pellowski, J. A., McGrath, J. M., Cusson, R. M., Gable, R. K., \& Fisher, J. D. (2017). Exclusive breast-feeding promotion among HIV-infected women in South Africa: An Information-Motivation-Behavioural Skills model-based pilot intervention. Public Health Nutrition, 1-10. doi:10.1017/S1368980016003657

Tyler, T. R., \& Schuller, R. A. (1991). Aging and attitude change. Journal of Personality and Social Psychology, 61, 689-697. 
Ugurlu, M., \& Yavan, T. (2016). The effectiveness of breastfeeding education: An integrative review. Journal of Behavioral Health, 5, 182-190. doi:10.5455/jbh.20160224063449

Unar-Munguía, M., Torres-Mejía, G., Colchero, M. A., \& González de Cosío, T. (2017).

Breastfeeding mode and risk of breast cancer: A dose-response meta-analysis. Journal of Human Lactation, 33, 422-434. doi:10.1177/0890334416683676

Urbaniak, G. C., \& Plous, S. (2013). Research randomizer (Version 4.0) [Computer software]. Retrieved from http://www.randomizer.org/

U.S. Department of Agriculture. (2015). About WIC-WIC at a glance. Retrieved from http://www.fns.usda.gov/wic/about-wic-wic-glance

U.S. Department of Health and Human Services (2011). The Surgeon General's call to action to support breastfeeding. Washington, DC: Office of the Surgeon General.

U.S. Preventive Services Task Force (2016). Primary care interventions to support breastfeeding: U.S. Preventive services task force recommendation statement. JAMA, 316, 1688-1693. doi:10.1001/jama.2016.14697

Vennemann, M. M., Bajanowski, T., Brinkmann, B., Jorch, G., Yücesan, K., Sauerland, C., \& Mitchell, E. A. (2009). Does breastfeeding reduce the risk of sudden infant death syndrome? Pediatrics, 123, e406-e410. doi:10.1542/peds.2008-2145

Victora, C. G., Bahl, R., Barros, A. J. D., França, G. V. A., Horton, S., Krasevec, J., Murch, S...Rollins, N. C. (2016). Breastfeeding in the 21st century: Epidemiology, mechanisms, and lifelong effect. The Lancet, 387, 475-490. doi:10.1016/s0140-6736(15)01024-7

Vinha, P. P., \& de Mello-Filho, F. V. (2017). Evidence of a Preventive Effect of Breastfeeding on Obstructive Sleep Apnea in Children and Adults. Journal of Human Lactation, 33, 448-453. doi:10.1177/0890334416682006 
Wagner, C. L., \& Greer, F. R. (2008). Prevention of rickets and Vitamin D deficiency in infants, children, and adolescents. Pediatrics, 122, 1142-1152. doi:10.1542/peds.2008-1862.

Wallenborn, J. T., Ihongbe, T., Rozario, S., \& Masho, S. W. (2017). Knowledge of breastfeeding recommendations and breastfeeding duration: A survival analysis on Infant Feeding Practices II. Breastfeeding Medicine, 12, 156-162. doi:10.1089/bfm.2016.0170

Wallis, A. B., Brinzaniuc, A., Chereches, R., Oprescu, F., Sirlincsn, E., David, I., ...\& Dungy, C. I. (2008). Reliability and validity of the Romanian version of a scale to measure infant feeding attitudes and knowledge. Acta Padiatrica, 97, 1194-1199. doi:10.1111/j.16512227.2008.00914.x

Wampold, B. E. (2015). How important are the common factors in psychotherapy? An update. World Psychiatry, 14, 270-277. doi:10.1002/wps.20238

Welch, G., Rose, G., \& Ernst, D. (2006). Motivational interviewing and diabetes: what is it, how is it used, and does it work?. Diabetes Spectrum, 19, 5-11. doi:10.2337/diaspect.19.1.5

Wiener, R., \& Wiener, M. A. (2011). Breastfeeding prevalence and distribution in the USA and Appalachia by rural and urban setting. Rural and Remote Health, 11, 1713.

Wight, N. E. (2015). Breastfeeding the NICU infant: What to expect. Clinical Obstetrics and Gynecology, 58, 840-854. doi:10.1097/GRF.0000000000000140

Wilhelm, S. L., Aguirre, T. M., Koehler, A. E., \& Rodehorst, T. K. (2015). Evaluating Motivational Interviewing to promote breastfeeding by rural Mexican-American mothers: The challenge of attrition. Issues in Comprehensive Pediatric Nursing, 38, 7-21. doi:10.3109/01460862.2014.971977 
Wilhelm, S. L., Rodehorst, T. K., Stepans, M. B. F., Hertzog, M., \& Berens, C. (2008). Influence of intention and self-efficacy levels on duration of breastfeeding for midwest rural mothers. Applied Nursing Research, 21, 123-130. doi:10.1016/j.apnr.2006.10.005

Wilhelm, S. L., Stepans, M. B. F., Hertzog, M., Callahan Rodehorst, T. K., \& Gardner, P. (2006). Motivational interviewing to promote sustained breastfeeding. Journal of Obstetric, Gynecologic, \& Neonatal Nursing, 35, 340-348.

doi:10.1177/0890334409352904

Wilson-Clay, B. (1996). Clinical uses of nipple shields. Journal of Human Lactation, 12, 279285.

Woodman, K., Bayne, F., \& MacDonald, A. (2014). The pragmatic use of evidence to inform breastfeeding peer support guidance for Scotland. The Lancet, 384, S88. doi:10.1016/S0140-6736(14)62214-5

Wooldridge, J., \& Hall, W. A. (2003). Posthospitalization breastfeeding patterns of moderately preterm infants. Journal of Perinatal Neonatal Nursing, 17, 50-64.

World Health Organization. (2009). Infant and young child feeding: Model chapter for textbooks for medical students and allied health professionals. Retrieved from https://www.ncbi.nlm.nih.gov/books/NBK148955/

World Health Organization. (2016). Exclusive breastfeeding. Retrieved from http://www.who.int/nutrition/topics/exclusive_breastfeeding/en/

World Health Organization. (2017a). Infant and young child feeding data by country. Retrieved from http://www.who.int/nutrition/databases/infantfeeding/countries/en/ 
World Health Organization. (2017b). National implementation of the baby-friendly hospital initiative by country. Retrieved from http://apps.who.int/iris/bitstream/ 10665/255197/1/9789241512381-eng.pdf?ua=1

Wouk, K., Tully, K. P., \& Labbok, M. H. (2017). Systematic review of evidence for BabyFriendly Hospital Initiative Step 3: Prenatal breastfeeding education. Journal of Human Lactation, 33, 50-82. doi:10.1177/0890334416679618

Yao, N., Matthews, S. A. and Hillemeier, M. M. (2012). White infant mortality in Appalachian states, 1976-1980 and 1996-2000: Changing patterns and persistent disparities. The Journal of Rural Health, 28, 174-182. doi:10.1111/j.1748-0361.2011.00385.x

Young, I. M. (2003). Breasted experience: The look and the feeling. In R. Weitz (Ed.), The politics of women's bodies (pp. 152-163). New York: Oxford University Press.

Zimmerman, D. R., \& Guttman, N. (2001). "Breast is best”: Knowledge among low-income mothers is not enough. Journal of Human Lactation, 17, 14-19.

doi: $10.1177 / 0890334401017$

Zunza, M., Cotton, M. F., Mbuagbaw, L., Lester, R., \& Thabane, L. (2017). Interactive weekly mobile phone text messaging plus motivational interviewing in promotion of breastfeeding among women living with HIV in South Africa: Study protocol for a randomized controlled trial. Trials, 18, 331. doi:10.1186/s13063-017-2079- 
Table 1

Interagency Group for Action on Breastfeeding Categories and Corresponding Items in the Postpartum Interview (Labbok \& Krasovec, 1990; Labbok \& Starling, 2012)

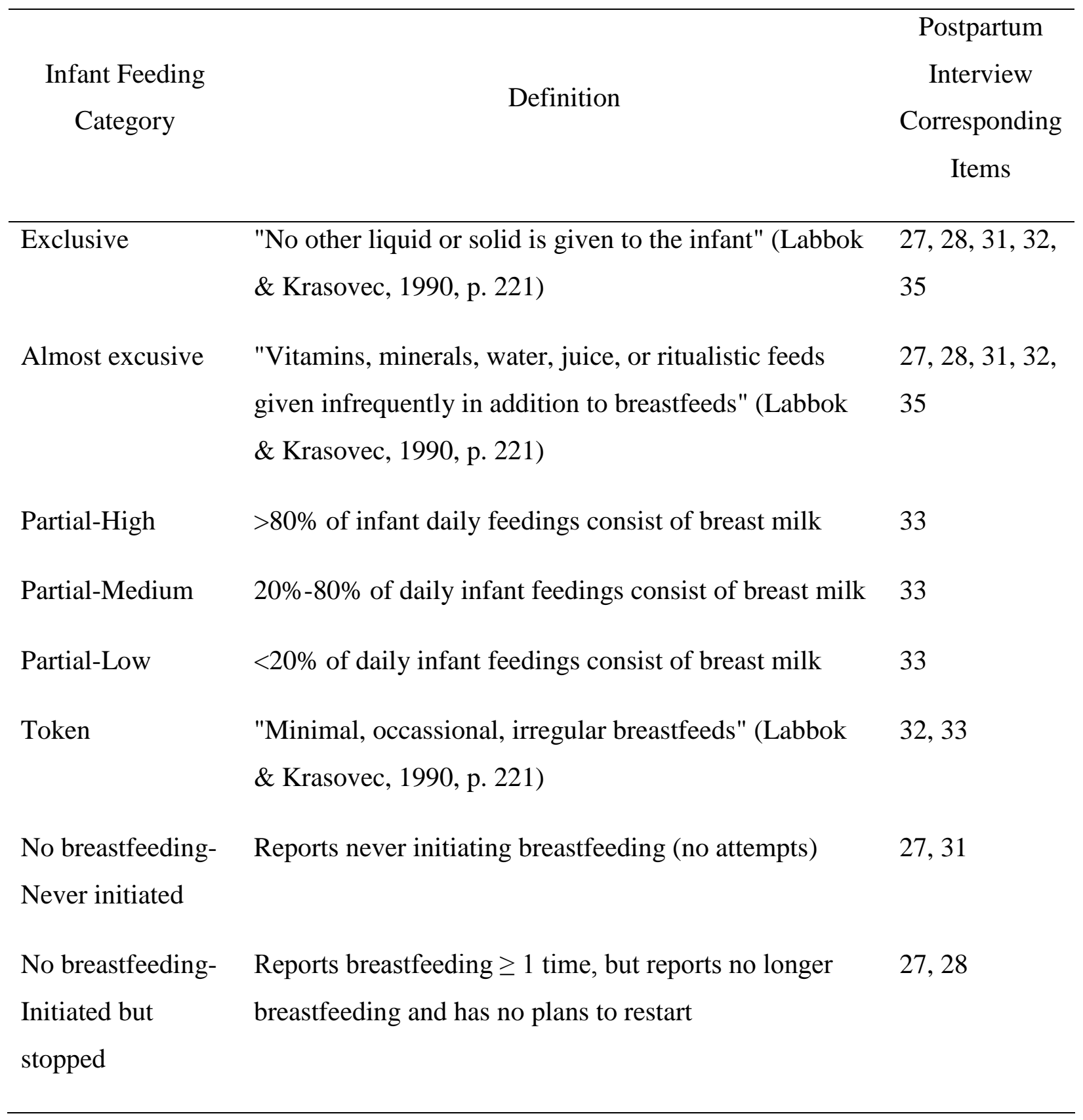

Note. In the present study, "Exclusive" breastfeeding includes infants receiving vitamins or medications recommended by a healthcare provider. "Partial-Medium" was further broken down into sub-categories of 20\%-39\% breastmilk, 40\%-59\% breastmilk, and 60\%-79\% breastmilk. 
Table 2

Total Number (and Percentage) for Recruitment and In-Person Session Information

\begin{tabular}{|c|c|c|c|}
\hline & $\begin{array}{l}\text { Overall } \\
(N=81)\end{array}$ & $\begin{array}{c}\text { MI } \\
(n=41)\end{array}$ & $\begin{array}{c}\mathrm{PE} \\
(n=40)\end{array}$ \\
\hline \multicolumn{4}{|l|}{ Recruitment Source } \\
\hline Word of mouth & $16(19.8)$ & $9(22.0)$ & $7(17.5)$ \\
\hline Clinics & $14(17.3)$ & $7(17.1)$ & $7(17.5)$ \\
\hline Facebook & $33(40.7)$ & $16(39.0)$ & $17(42.5)$ \\
\hline WVU Listservs & $9(11.1)$ & $5(12.2)$ & $4(10.0)$ \\
\hline Craigslist & $1(1.2)$ & $1(2.4)$ & - \\
\hline WIC & $5(6.2)$ & $2(4.9)$ & $3(7.5)$ \\
\hline Prenatal Yoga & $3(3.7)$ & $1(2.4)$ & $2(5.0)$ \\
\hline \multicolumn{4}{|l|}{ Location of Visit } \\
\hline Participant Home & $38(46.9)$ & $14(34.2)$ & $24(60.0)$ \\
\hline Quin Curtis Center & $35(43.2)$ & $25(61.0)$ & $10(25.0)$ \\
\hline Other & $8(9.9)$ & $2(4.9)$ & $6(15.0)$ \\
\hline \multicolumn{4}{|l|}{ Therapist } \\
\hline A & $51(63.0)$ & $26(63.4)$ & $25(62.5)$ \\
\hline $\mathrm{B}$ & $30(37.0)$ & $15(36.6)$ & $15(37.5)$ \\
\hline
\end{tabular}

Note. $\mathrm{MI}=$ Motivational Interviewing; $\mathrm{PE}=$ Psychoeducation. "Clinics" refer to WVU Family Medicine, WVU Obstetrics and Gynecology, and the Chestnut Ridge Center COAT Clinics. 
Table 3

Total Number/Mean (and Percentage) for Participant Residence Location

\begin{tabular}{|c|c|c|c|c|}
\hline & & $\begin{array}{l}\text { Overall } \\
(N=81)\end{array}$ & $\begin{array}{c}\text { MI } \\
(n=41)\end{array}$ & $\begin{array}{c}\mathrm{PE} \\
(n=40)\end{array}$ \\
\hline \multicolumn{5}{|l|}{ State } \\
\hline \multicolumn{5}{|c|}{ County } \\
\hline \multirow[t]{2}{*}{ Ohio } & & $1(1.2)$ & $1(2.4)$ & - \\
\hline & Belmont & $1(1.2)$ & $1(2.4)$ & - \\
\hline \multirow[t]{5}{*}{ Pennsylvania } & & $11(14.6)$ & $6(14.6)$ & $5(12.5)$ \\
\hline & Allegheny & $4(4.9)$ & $2(4.9)$ & $2(5.0)$ \\
\hline & Fayette & $1(1.2)$ & $1(2.4)$ & \\
\hline & Green & $5(6.2)$ & $2(4.9)$ & $3(7.5)$ \\
\hline & Washington & $1(1.2)$ & $1(2.4)$ & - \\
\hline \multirow[t]{9}{*}{ West Virginia } & & $68(84.0)$ & 34 (82.9) & $35(87.5)$ \\
\hline & Harrison & $5(6.2)$ & $5(12.2)$ & $1(2.5)$ \\
\hline & Kanawha & $2(2.5)$ & $1(2.4)$ & $1(2.5)$ \\
\hline & Marion & $10(12.4)$ & $5(12.2)$ & $5(12.5)$ \\
\hline & Marshall & $1(1.2)$ & - & $1(2.5)$ \\
\hline & Monongalia & $44(54.3)$ & $22(53.7)$ & $22(55.0)$ \\
\hline & Preston & $2(2.5)$ & $1(2.4)$ & $1(2.5)$ \\
\hline & Taylor & $2(2.5)$ & - & $2(5.0)$ \\
\hline & Upshur & $3(3.7)$ & $1(2.4)$ & $2(5.0)$ \\
\hline
\end{tabular}

Note. $\mathrm{MI}=$ Motivational Interviewing; $\mathrm{PE}=$ psychoeducation. 
Table 4

Summary of Missing Data per Participant

\begin{tabular}{|c|c|c|c|c|c|c|}
\hline Participant & Pre-IIFAS & Pre-IFKF & Post-IFKF & Post-KIDI & $\begin{array}{l}\text { Post- } \\
\text { BBAS }\end{array}$ & $\begin{array}{l}\text { Postpartum } \\
\text { Phone Call }\end{array}$ \\
\hline 1 & & & $\mathrm{X}$ & & & \\
\hline 18 & & & & & & $X$ \\
\hline 36 & $X$ & & & & & \\
\hline 47 & $X$ & & & & & \\
\hline 50 & & & & $X$ & & \\
\hline 51 & & & & & & $X$ \\
\hline 59 & $X$ & & & & & \\
\hline 61 & & & & $X$ & & \\
\hline 64 & & & & $X$ & & \\
\hline 66 & $X$ & & & & & \\
\hline 78 & & $X$ & $X$ & & & \\
\hline 80 & & & & $X$ & & \\
\hline 81 & & & & & $\mathrm{X}$ & \\
\hline Total/ & & & & & & \\
\hline $\begin{array}{l}\text { Percentage } \\
\text { of Sample }\end{array}$ & $4 / 4.94$ & $1 / 1.23$ & $2 / 2.47$ & $4 / 4.94$ & $1 / 1.23$ & $2 / 2.47$ \\
\hline
\end{tabular}

Note. Listwise deletion was used for self-report measures, such that participant data for a measure was considered "missing" if she skipped $\geq 1$ items within the measure. 
Table 5

Skewness and Kurtosis of Dependent Variables

\begin{tabular}{|c|c|c|}
\hline Dependent Measure & Skewness $(S E)$ & Kurtosis $(S E)$ \\
\hline KIDI—Pre & $-0.06(0.27)$ & $-0.50(0.53)$ \\
\hline KIDI-Post & $-0.82(0.27)$ & $0.70(0.54)$ \\
\hline IIFAS_-Pre & $-0.29(0.27)$ & $1.18(0.53)$ \\
\hline IIFAS_Post & $-0.38(0.27)$ & $1.23(0.53)$ \\
\hline IFKF_-Pre & $-0.60(0.27)$ & $-0.39(0.53)$ \\
\hline IFKF_-Post* & $-1.84(0.27)$ & $4.90(0.53)$ \\
\hline $\begin{array}{l}\text { Transformed IFKF_-Post } \\
\text { (Square root) }\end{array}$ & $0.88(0.27)$ & $1.94(0.54)$ \\
\hline BBAS-Pre & $-0.94(0.27)$ & $0.44(0.53)$ \\
\hline BBAS_Post & $-1.02(0.27)$ & $.61(0.53)$ \\
\hline Perceived behavioral control—Pre & $-1.19(0.27)$ & $1.47(0.53)$ \\
\hline Perceived behavioral control —Post & $-1.60(0.27)$ & $-1.60(0.53)$ \\
\hline Importance-Pre* & $-1.58(0.27)$ & $2.38(0.53)$ \\
\hline $\begin{array}{l}\text { Transformed Importance-Pre } \\
\text { (Square root) }\end{array}$ & $0.88(0.27)$ & $0.11(0.53)$ \\
\hline Importance-_Post* & $-2.02(0.27)$ & $4.06(0.53)$ \\
\hline $\begin{array}{l}\text { Transformed Importance - Post } \\
\text { (Square root) }\end{array}$ & $1.26(0.27)$ & $1.24(0.53)$ \\
\hline Intended Length of breastfeeding (months) - Pre & $0.46(0.27)$ & $1.54(0.53)$ \\
\hline Intended Length of breastfeeding (months)_-Post & $0.58(0.27)$ & $1.46(0.53)$ \\
\hline Perceived behavioral control-PP & $-0.90(0.29)$ & $0.74(0.57)$ \\
\hline Importance-PP & $-1.32(0.29)$ & $1.64(0.57)$ \\
\hline Intended length of breastfeeding (months)—PP* & $0.79(0.29)$ & $1.08(0.57)$ \\
\hline
\end{tabular}

Note . Pre = immediately before intervention; Post = immediately after intervention; $\mathrm{PP}=$ one month postpartum.

*Skewness/kurtosis values $>2$ and therefore was subject to transformation. 
Table 6

Correlations Among Dependent Variable Instruments

\begin{tabular}{|c|c|c|c|c|c|c|c|c|}
\hline & $\begin{array}{c}\text { KIDI- } \\
\text { Pre }\end{array}$ & $\begin{array}{l}\text { KIDI- } \\
\text { Post }\end{array}$ & $\begin{array}{c}\text { IIFAS- } \\
\text { Pre }\end{array}$ & $\begin{array}{c}\text { IIFAS- } \\
\text { Post }\end{array}$ & $\begin{array}{c}\text { IFKF- } \\
\text { Pre }\end{array}$ & $\begin{array}{c}\text { IFKF- } \\
\text { Post }\end{array}$ & $\begin{array}{c}\text { BBAS- } \\
\text { Pre }\end{array}$ & $\begin{array}{c}\text { BBAS- } \\
\text { Pre }\end{array}$ \\
\hline KIDI- & 1.00 & $.60 * *$ & .04 & -.01 & .16 & $.27 *$ & -.01 & -.01 \\
\hline \multicolumn{9}{|l|}{ Pre } \\
\hline KIDI- & & 1.00 & -.06 & -.19 & -.06 & .10 & -.08 & -.08 \\
\hline \multicolumn{9}{|l|}{ Post } \\
\hline IIFAS- & & & 1.00 & $.74 * *$ & $.45^{* *}$ & .23 & $.43 * *$ & $.42 * *$ \\
\hline \multicolumn{9}{|l|}{ Pre } \\
\hline IIFAS- & & & & 1.00 & $.26^{*}$ & $.45^{*}$ & $.37^{*}$ & $.45 * *$ \\
\hline \multicolumn{9}{|l|}{ Post } \\
\hline IFKF- & & & & & 1.00 & $.52 * *$ & .18 & .16 \\
\hline \multicolumn{9}{|l|}{ Pre } \\
\hline IFKF- & & & , & & & 1.00 & .17 & .21 \\
\hline \multicolumn{9}{|l|}{ Post } \\
\hline BBAS- & & & & & & & 1.00 & $.89 * *$ \\
\hline \multicolumn{9}{|l|}{ Pre } \\
\hline BBAS- & & & & & & & & 1.00 \\
\hline Post & & & & & & & & \\
\hline
\end{tabular}

Note. KIDI $=$ Knowledge of Infant Development Inventory; IIFAS = Iowa Infant Feeding

Attitudes Scale; IFKF = Infant Feeding Knowledge Form; BBAS = Brief Breastfeeding Attitudes Scale.

${ }^{*} p \leq .05, * * p \leq .01$ 
Table 7

Total Number/Mean (and Percentages or Standard Deviations) and Statistical Test Results for Baseline Demographic Characteristics

\begin{tabular}{lccccc}
\hline & $\begin{array}{c}\text { Overall } \\
(N=81)\end{array}$ & $\begin{array}{c}\text { MI } \\
(n=41)\end{array}$ & $\begin{array}{c}\text { PE } \\
(n=40)\end{array}$ & $t / \chi^{2}$ & $P$ \\
\hline Age, Mean (SD) & $27.86(5.24)$ & $27.51(6.04)$ & $28.23(4.31)$ & 0.61 & .544 \\
Household annual income (\$), Mean & 83,234 & 81,396 & 72,478 & - & \\
(SD) & $(72,509)$ & $(50,813)$ & $(47,461)$ & 0.78 & .437
\end{tabular}

Household annual income, range

$\begin{array}{rccc}\text { Less than } \$ 10,000 & 4(4.9) & 2(4.9) & 2(5.0) \\ \$ 10,000-\$ 14,999 & 3(3.7) & 1(2.4) & 2(5.0) \\ \$ 15,000-\$ 24,999 & 5(6.2) & 2(4.9) & 3(7.5) \\ \$ 25,000-\$ 34,999 & 8(9.9) & 4(9.8) & 4(10.0) \\ \$ 35,000-\$ 49,999 & 5(6.2) & 2(4.9) & 3(7.5) \\ \$ 50,000-\$ 74,999 & 11(13.6) & 5(12.2) & 6(15.0) \\ \$ 75,000-\$ 99,999 & 17(21.0) & 11(26.8) & 6(15.0) \\ \geq \$ 100,000 & 25(30.9) & 13(31.7) & 12(30.0)\end{array}$

$\begin{array}{llllll}\text { Employed } & 69(76.5) & 36(78.0) & 33(82.5) & 0.17 & .753 \\ \text { Earns } \geq 50 \% \text { household income } & 34(42.0) & 15(36.6) & 19(47.5) & 1.47 & .332 \\ \text { Worked } \geq 35 \text { hours/week } & 50(60.2) & 28(68.3) & 22(55.0) & 1.39 & .323\end{array}$

Plans to work during baby's $1^{\text {st }}$ year

$65(80.3) \quad 33(80.5) \quad 32(80.0) \quad 0.29 \quad .770$

Total weeks paid maternity leave,

Mean (SD)

$2.44(3.40) \quad 2.38(2.78) \quad 2.52(4.07) \quad 0.15 \quad .886$

$\begin{array}{llllll}\text { Planned maternity leave }>9 \text { weeks } \quad 41(50.6) & 22(53.7) & 19(47.5) & 0.09 & .806\end{array}$

Perceived employer to be "very

supportive" of breastfeeding

$\begin{array}{lllll}32(39.5) & 19(46.3) & 13(32.5) & 1.01 & .437\end{array}$ 
Table 7 continued

\begin{tabular}{|c|c|c|c|c|c|}
\hline & $\begin{array}{l}\text { Overall } \\
(N=81)\end{array}$ & $\begin{array}{c}\text { MI } \\
(n=41)\end{array}$ & $\begin{array}{c}\mathrm{PE} \\
(n=40)\end{array}$ & $t / \chi^{2}$ & $P$ \\
\hline Hispanic ethnicity (Participant) & $7(8.6)$ & $6(14.6)$ & $1(2.5)$ & 3.78 & .109 \\
\hline \multicolumn{6}{|l|}{ Race-Participant } \\
\hline African American & $3(3.7)$ & - & $3(7.5)$ & & \\
\hline Asian & $1(1.2)$ & - & $1(2.5)$ & & \\
\hline Hispanic & $3(3.7)$ & $2(4.9)$ & $1(2.5)$ & & \\
\hline Multi-racial & $1(1.2)$ & - & $1(2.5)$ & & \\
\hline White & $72(88.9)$ & $38(92.7)$ & $34(85.0)$ & & \\
\hline Other & $1(1.2)$ & $1(2.4)$ & - & & \\
\hline Hispanic ethnicity (Baby's father) & $6(7.4)$ & $4(9.8)$ & $2(5.0)$ & 0.67 & .675 \\
\hline \multicolumn{6}{|l|}{ Race_-Baby's Father } \\
\hline African American & $2(2.5)$ & $2(4.9)$ & - & & \\
\hline Asian & $1(1.23)$ & - & $1(2.5)$ & & \\
\hline Hispanic & $3(3.7)$ & $1(2.4)$ & $2(5.0)$ & & \\
\hline Multi-racial & - & - & - & & \\
\hline White & $73(90.1)$ & $37(90.2)$ & $36(90.0)$ & & \\
\hline Has health insurance & $80(98.8)$ & $41(100.0)$ & $39(97.5)$ & 1.04 & .494 \\
\hline Has Medicaid & $18(22.2)$ & $8(19.5)$ & $10(25.0)$ & 0.43 & .597 \\
\hline $\begin{array}{l}\text { Years Education-Participant, } \\
\text { Mean (SD) }\end{array}$ & $\begin{array}{l}16.05 \\
(3.01)\end{array}$ & $\begin{array}{l}16.09 \\
(3.00)\end{array}$ & $16.01(3.05)$ & -0.11 & .914 \\
\hline $\begin{array}{l}\text { Years Education-Baby's father, } \\
\text { Mean (SD) }\end{array}$ & $\begin{array}{l}15.88 \\
(3.37)\end{array}$ & $\begin{array}{l}15.93 \\
(2.81)\end{array}$ & $15.83(3.90)$ & -0.14 & .893 \\
\hline Enrolled in WIC & $16(19.8)$ & $6(14.6)$ & $10(25.0)$ & & \\
\hline \# Children in home, Mean (SD) & $0.41(0.72)$ & $0.39(0.75)$ & $0.43(0.70)$ & 0.22 & .830 \\
\hline
\end{tabular}

Note $. \mathrm{MI}=$ Motivational Interviewing, $\mathrm{PE}=$ psychoeducation. Statistical tests for categorical income, participant race, and race of baby's father not calculated due to small cell sizes. Long term relationship $=$ married or cohabitating with partner. Length of maternity leave referred to uncomplicated vaginal delivery. 
Table 8

Total Number/Mean (and Percentages or Standard Deviations) and Statistical Test Results for Baseline Breastfeeding-related Experiences and Perceptions

\begin{tabular}{|c|c|c|c|c|c|}
\hline & $\begin{array}{l}\text { Overall } \\
(N=81)\end{array}$ & $\begin{array}{c}\mathrm{MI} \\
(n=41)\end{array}$ & $\begin{array}{c}\mathrm{PE} \\
(n=40)\end{array}$ & $t / \chi^{2}$ & $p$ \\
\hline Breastfed as baby (participant) & $53(65.4)$ & $29(70.7)$ & $24(60.0)$ & 1.96 & .220 \\
\hline \multicolumn{6}{|l|}{ Perceived others to favor exclusive } \\
\hline \multicolumn{6}{|l|}{ breastfeeding } \\
\hline Baby's father & $45(55.6)$ & $21(51.2)$ & $24(60.0)$ & 0.63 & .505 \\
\hline Baby's maternal grandmother & $38(46.9)$ & $25(61.0)$ & $18(45.0)$ & 1.80 & .255 \\
\hline Baby's paternal grandmother & $37(45.7)$ & $20(48.8)$ & $17(42.5)$ & 0.80 & .497 \\
\hline Prenatal healthcare provider & $48(59.3)$ & $23(56.1)$ & $25(62.5)$ & 0.53 & .501 \\
\hline \multirow{2}{*}{\multicolumn{6}{|c|}{$\begin{array}{l}\text { Importance of others' opinions, Mean } \\
\text { (SD) }\end{array}$}} \\
\hline & & & & & \\
\hline Baby's father & $3.56(0.81)$ & $3.68(0.72)$ & $3.43(0.87)$ & -1.45 & .151 \\
\hline Baby's maternal grandmother & $3.05(0.13)$ & $2.98(1.17)$ & $3.13(1.09)$ & 0.59 & .861 \\
\hline Baby's paternal grandmother & $2.42(1.07)$ & $2.42(1.13)$ & $2.41(1.02)$ & -0.04 & .965 \\
\hline Prenatal healthcare provider & $3.17(0.99)$ & $3.28(1.04)$ & $3.05(0.94)$ & -1.00 & .320 \\
\hline Ever breastfed before & $21(25.9)$ & $12(29.3)$ & $9(22.5)$ & 0.48 & .614 \\
\hline Vicarious breastfeeding, Mean (SD) & $2.18(0.85)$ & $2.20(0.91)$ & $2.16(0.79)$ & -0.22 & .828 \\
\hline
\end{tabular}

Note. $\mathrm{MI}=$ Motivational Interviewing, $\mathrm{PE}=$ psychoeducation. Importance of others' opinions was ranked on a scale from $1=$ not at all important, $2=$ not very important, $3=$ somewhat important, 4 = very important. Vicarious breastfeeding experience was measured with the item "How many of your friends and relatives have breastfed their babies?" $0=$ None, $1=1-2,2=3$ $5,4=>5$. 
Table 9

Total Number/Mean (and Percentages or Standard Deviations) and Statistical Test Results for Baseline Health and Mental Health Variables

\begin{tabular}{|c|c|c|c|c|c|}
\hline & $\begin{array}{l}\text { Overall } \\
(N=81)\end{array}$ & $\begin{array}{c}\text { MI } \\
(n=41)\end{array}$ & $\begin{array}{c}\text { PE } \\
(n=40)\end{array}$ & $t / \chi^{2}$ & $p$ \\
\hline Body Mass Index, Mean (SD) & $\begin{array}{l}28.17 \\
(8.29)\end{array}$ & $\begin{array}{l}27.97 \\
(7.97)\end{array}$ & $\begin{array}{l}28.37 \\
(8.70)\end{array}$ & 0.213 & .832 \\
\hline Pregnancy complications & $21(25.9)$ & $11(26.8)$ & $10(25.0)$ & 0.10 & .804 \\
\hline Gestational diabetes & $10(12.3)$ & $6(14.6)$ & $4(10.0)$ & 0.40 & .737 \\
\hline High blood pressure & $3(3.7)$ & - & $3(7.5)$ & 3.19 & .116 \\
\hline Smokes cigarettes & $5(6.2)$ & $1(2.4)$ & $4(10.0)$ & 2.00 & .201 \\
\hline $\begin{array}{l}\text { Among smokers, total cigarettes/day, } \\
\text { Mean (SD) }\end{array}$ & $\begin{array}{r}12.40 \\
(15.84)\end{array}$ & $10.00(\mathrm{n} / \mathrm{a})$ & $\begin{array}{r}13.00 \\
(18.22)\end{array}$ & 0.15 & .892 \\
\hline Weeks pregnant, Mean (SD) & $\begin{array}{l}31.78 \\
(4.32)\end{array}$ & $\begin{array}{l}31.65 \\
(4.41)\end{array}$ & $\begin{array}{l}32.00 \\
(4.42)\end{array}$ & 0.45 & .651 \\
\hline Primipara & $56(69.1)$ & $28(68.3)$ & $28(70.0)$ & 0.03 & 1.00 \\
\hline Had specific birth plan & $61(75.3)$ & $29(70.7)$ & $32(80.0)$ & 0.94 & .441 \\
\hline \multicolumn{6}{|l|}{ Prenatal healthcare provider } \\
\hline $\begin{array}{r}\text { Medical doctor } \\
\text { Midwife }\end{array}$ & $\begin{array}{l}60(74.1) \\
20(24.7)\end{array}$ & $\begin{array}{l}34(82.9) \\
13(31.7)\end{array}$ & $\begin{array}{l}26(65.0) \\
7(17.5)\end{array}$ & 2.82 & .123 \\
\hline \# previous pregnancies, Mean (SD) & $0.77(1.04)$ & $0.68(0.85)$ & $0.85(1.21)$ & 0.72 & .473 \\
\hline \# previous live births, Mean (SD) & $0.43(0.74)$ & $0.42(0.71)$ & $0.45(0.78)$ & 0.21 & .831 \\
\hline Unplanned pregnancy & $30(37.0)$ & $17(41.5)$ & $13(32.5)$ & 0.70 & .492 \\
\hline History of miscarriage & $15(18.5)$ & $6(14.6)$ & $9(22.5)$ & 0.83 & .404 \\
\hline History of abortion & $5(6.2)$ & $4(9.8)$ & $1(2.5)$ & 1.84 & .359 \\
\hline Ever been in therapy & $36(44.4)$ & $18(43.9)$ & $18(45.0)$ & 0.01 & 1.00 \\
\hline History of trauma (excluding abuse) & $3(3.7)$ & $1(2.4)$ & $2(5.0)$ & 0.43 & .606 \\
\hline History of abuse & $22(27.2)$ & $4(9.8)$ & $7(17.5)$ & 1.31 & .343 \\
\hline $\begin{array}{l}\text { Perinatal Anxiety Screening Scale, } \\
\text { Mean (SD) }\end{array}$ & $\begin{array}{c}19.16 \\
(14.16)\end{array}$ & $\begin{array}{r}18.60 \\
(12.39)\end{array}$ & $\begin{array}{r}19.78 \\
(16.06)\end{array}$ & 0.36 & .720 \\
\hline
\end{tabular}


Table 9 continued

\begin{tabular}{lccccc}
\hline & $\begin{array}{c}\text { Overall } \\
(N=81)\end{array}$ & $\begin{array}{c}\text { MI } \\
(n=41)\end{array}$ & $\begin{array}{c}\text { PE } \\
(n=40)\end{array}$ & $t / \chi^{2}$ & $p$ \\
\hline History of anxiety & $23(28.4)$ & $12(29.3)$ & $11(27.5)$ & 0.06 & 1.00 \\
History of depression & $22(27.2)$ & $12(29.3)$ & $10(25.0)$ & 0.25 & .803 \\
History of other mental disorder & $4(4.9)$ & $2(4.9)$ & $2(5.0)$ & 0.003 & 1.00 \\
\hline
\end{tabular}

Note. $\mathrm{MI}=$ Motivational Interviewing; $\mathrm{PE}=$ psychoeducation. Specific pregnancy complications, past medical histories, and specific medications of participants available in Appendix Y. 
Table 10

Total Number/Mean (and Percentages or Standard Deviations) and Statistical Test Results for One Month Postpartum Baby and Birth Variables

\begin{tabular}{lccccc}
\hline & $\begin{array}{c}\text { Overall } \\
(N=79)\end{array}$ & $\begin{array}{c}\text { MI } \\
(n=40)\end{array}$ & $\begin{array}{c}\text { PE } \\
(n=39)\end{array}$ & $t / \chi^{2}$ & $P$ \\
\hline Took breastfeeding class during & $36(45.6)$ & $17(42.5)$ & $19(48.7)$ & 0.31 & .654 \\
$\begin{array}{l}\text { pregnancy } \\
\text { Gestational weeks, Mean (SD) }\end{array}$ & 38.75 & $39.44(1.32)$ & 38.04 & & \\
& $(4.45)$ & & $(6.16)$ & & \\
Cesarean section & $24(30.4)$ & $12(30.0)$ & $12(30.78)$ & 0.01 & 1.00 \\
Epidural & $62(78.5)$ & $31(77.5)$ & $31(79.5)$ & 0.20 & .781 \\
Delivery location & & & & \\
$\quad$ Ruby Memorial Hospital & $42(53.2)$ & $20(50.0)$ & $22(56.4)$ & & \\
Monongalia General Hospital & $19(24.1)$ & $11(27.5)$ & $8(20.5)$ & & \\
$\quad$ United Hospital Center & $5(6.3)$ & $2(5.0)$ & $3(7.7)$ & &
\end{tabular}

Birth attendant

$\begin{array}{rccccc}\text { Medical doctor } & 60(76.0) & 31(77.5) & 29(74.4) & 0.11 & .797\end{array}$

$\begin{array}{lllll}\text { Skin to skin ("Kangaroo Care") } & 67(84.8) & 36(90.0) & 31(79.5) & 1.69\end{array}$

"Rooming-in" all nights in hospital $\quad 68(86.1) \quad 36(90.0) \quad 32(82.1) \quad 1.04 \quad 348$

$\begin{array}{lccccc}\text { APGAR score, Mean (SD) } & 8.15 & 8.09(1.04) & 8.19 & 0.24 & .816\end{array}$

$\begin{array}{llllll}\text { Sex of baby }(\% \text { male }) & 40(50.6) & 21(52.5) & 19(48.7) & 0.32 & .655\end{array}$

$\begin{array}{llllll}\text { Baby length (inches), Mean (SD) } & 20.18 & 20.42 & 19.95 & -1.95 & .061\end{array}$

$\begin{array}{lccccc}\text { Baby weight (pounds), Mean (SD) } & 7.62 & 7.77(1.16) & 7.47 & -1.23 & .224\end{array}$

$\begin{array}{llllll}\text { NICU admission } & 7(8.9) & 1(2.5) & 6(15.4) & 4.06 & .057\end{array}$ 
Table 10 continued

\begin{tabular}{|c|c|c|c|c|c|}
\hline & $\begin{array}{l}\text { Overall } \\
(N=79)\end{array}$ & $\begin{array}{c}\text { MI } \\
(n=40)\end{array}$ & $\begin{array}{c}\mathrm{PE} \\
(n=39)\end{array}$ & $t / \chi^{2}$ & $P$ \\
\hline \# Nights in hospital after birth & $2.56(1.21)$ & $2.33(0.53)$ & $\begin{array}{c}2.80 \\
(1.61)\end{array}$ & 1.70 & .093 \\
\hline Baby has long-term health problem & $1(1.3)$ & - & $1(2.6)$ & 0.99 & 1.00 \\
\hline Baby received formula in hospital & $26(32.9)$ & $10(25.0)$ & $16(41.0)$ & 2.08 & .230 \\
\hline Baby Vitamin D supplement & $22(27.2)$ & $16(39.0)$ & $6(15.0)$ & 5.96 & $.023^{*}$ \\
\hline $\begin{array}{l}\text { Total breastfeeding problems, } \\
\text { Mean (SD) }\end{array}$ & $2.35(1.70)$ & $2.32(1.42)$ & $\begin{array}{c}2.82 \\
(1.81)\end{array}$ & 0.81 & .422 \\
\hline $\begin{array}{l}\text { Received breastfeeding support in } \\
\text { hospital }\end{array}$ & $75(94.9)$ & $36(90.0)$ & $39(100.0)$ & 3.12 & .240 \\
\hline \multicolumn{6}{|l|}{ Source of support (could select $>1$ ) } \\
\hline Lactation consultant & $55(69.6)$ & $27(67.5)$ & $28(71.8)$ & & \\
\hline Nurse or midwife & $22(27.9)$ & $15(37.5)$ & $7(18.0)$ & & \\
\hline Doctor & $5(6.3)$ & $2(5.0)$ & $3(7.7)$ & & \\
\hline Other & $23(29.1)$ & $14(35.0)$ & $9(23.1)$ & & \\
\hline \multicolumn{6}{|l|}{$\begin{array}{l}\text { Perceived others to favor exclusive } \\
\text { breastfeeding }\end{array}$} \\
\hline Hospital staff & $62(84.8)$ & 29 (72.5) & $33(84.62)$ & 1.91 & .250 \\
\hline Participant's doctor & $45(57.0)$ & $22(55.0)$ & $23(58.97)$ & 0.13 & .821 \\
\hline Baby's pediatrician & $45(57.0)$ & $24(60.0)$ & $21(53.85)$ & 0.18 & .819 \\
\hline Breastfeeding valence & $3.47(1.11)$ & $3.60(1.11)$ & $\begin{array}{c}3.32 \\
(1.11)\end{array}$ & -1.09 & .279 \\
\hline Postpartum depression & $9(11.39)$ & $4(10.0)$ & $5(12.82)$ & 0.16 & .737 \\
\hline Smokes cigarettes & $5(6.33)$ & $2(5.0)$ & $3(7.69)$ & 0.24 & .675 \\
\hline $\begin{array}{l}\text { Among smokers, total } \\
\text { cigarettes/day, Mean (SD) }\end{array}$ & 8.7 (7.55) & $\begin{array}{l}10.00 \\
(0.00)\end{array}$ & $\begin{array}{c}7.83 \\
(10.54)\end{array}$ & -0.28 & .801 \\
\hline
\end{tabular}


Table 10 continued

\begin{tabular}{lccccc}
\hline & Overall & MI & PE & & \\
& $(N=79)$ & $(n=40)$ & $(n=39)$ & & \\
\hline Returned to work & $3(3.8)$ & $1(2.5)$ & $2(5.1)$ & 0.40 & 0.610 \\
\hline
\end{tabular}

Note. $\mathrm{MI}=$ Motivational Interviewing $; \mathrm{PE}=$ psychoeducation; $\mathrm{NICU}=$ Neonatal intensive care unit. Statistical tests not calculated for delivery location and type of breastfeeding assistance due to small cell sizes. Only about one-third of the sample reported knowing baby's APGAR score $(n=27,34.2 \%)$. "Breastfeeding class" refers to a formal class outside of routine prenatal visits that was solely or partially focused on breastfeeding. Breastfeeding valence measured with item, "how much did you like breastfeeding on a scale of $1-5,1=$ very much disliked it, $5=$ really liked it.

$* p \leq .05$ 
Table 11

Total Number/Mean (and Percentages or Standard Deviations) and Statistical Test Results for Baseline Dependent Variables

\begin{tabular}{|c|c|c|c|c|c|}
\hline & $\begin{array}{l}\text { Overall } \\
(N=81)\end{array}$ & $\begin{array}{c}\text { MI } \\
(n=41)\end{array}$ & $\begin{array}{c}\text { PE } \\
(n=40)\end{array}$ & $t \chi^{2}$ & $p$ \\
\hline IFKF & $\begin{array}{l}10.15 \\
(1.34)\end{array}$ & $\begin{array}{l}10.07 \\
(1.49)\end{array}$ & $\begin{array}{l}10.23 \\
(1.18)\end{array}$ & 0.52 & .603 \\
\hline IIFAS & $\begin{array}{l}63.79 \\
(7.42)\end{array}$ & $\begin{array}{l}63.97 \\
(6.31)\end{array}$ & $\begin{array}{l}63.61 \\
(8.50)\end{array}$ & -0.22 & .829 \\
\hline KIDI & $\begin{array}{c}63.09 \\
(10.91)\end{array}$ & $\begin{array}{c}61.83 \\
(10.47)\end{array}$ & $\begin{array}{c}64.37 \\
(11.33)\end{array}$ & 1.05 & .297 \\
\hline BBAS & $\begin{array}{l}17.48 \\
(2.32)\end{array}$ & $\begin{array}{l}17.98 \\
(2.02)\end{array}$ & $\begin{array}{l}16.98 \\
(2.52)\end{array}$ & -1.98 & .052 \\
\hline $\begin{array}{l}N(\%) \text { intending to exclusively } \\
\text { breastfeed }\end{array}$ & $73(90.1)$ & $\begin{array}{c}38 \\
(92.7)\end{array}$ & $\begin{array}{c}35 \\
(87.5)\end{array}$ & 1.11 & .555 \\
\hline $\begin{array}{l}\text { Duration of intended } \\
\text { breastfeeding in months }\end{array}$ & $\begin{array}{l}10.65 \\
(4.31)\end{array}$ & $\begin{array}{l}10.42 \\
(4.62)\end{array}$ & $\begin{array}{l}10.90 \\
(4.02)\end{array}$ & 0.50 & .616 \\
\hline $\begin{array}{l}\text { Importance of breastfeeding for } \\
\text { intended duration }\end{array}$ & $\begin{array}{c}8.16 \\
(2.28)\end{array}$ & $\begin{array}{c}8.54 \\
(1.90)\end{array}$ & $7.78(2.59)$ & -1.51 & .134 \\
\hline $\begin{array}{l}\text { Perceived behavioral control of } \\
\text { breastfeeding for intended } \\
\text { duration }\end{array}$ & $\begin{array}{c}7.44 \\
(2.36)\end{array}$ & $\begin{array}{c}7.93 \\
(1.86)\end{array}$ & $6.95(2.72)$ & -1.89 & .062 \\
\hline
\end{tabular}

Note. KIDI: Knowledge of Infant Development Inventory; IIFAS: Iowa Infant Feeding Attitudes Scale; IFKF: Infant Feeding Knowledge Form; BBAS: Brief Breastfeeding Attitudes Scale 
Table 12

Total Number/Mean (and Percentages or Standard Errors of the Mean) and Statistical Results for One Month Postpartum Feeding Characteristics

\begin{tabular}{|c|c|c|c|c|c|}
\hline Feeding Characteristic & $\begin{array}{l}\text { Overall } \\
(N=79)\end{array}$ & $\begin{array}{c}\text { MI } \\
(n=40)\end{array}$ & $\begin{array}{c}\mathrm{PE} \\
(n=39)\end{array}$ & $t / \chi^{2}$ & $p$ \\
\hline Breastfeeding, initiated & $77(97.5)$ & $\begin{array}{c}40 \\
(100.0)\end{array}$ & $37(94.9)$ & 2.11 & .241 \\
\hline Breastfeeding, any current & $69(85.2)$ & $38(92.7)$ & $31(79.5)$ & 4.30 & $.048^{*}$ \\
\hline Breastfeeding, exclusive & $43(53.1)$ & $23(56.1)$ & $20(51.3)$ & 0.31 & .654 \\
\hline Fed expressed milk in past week & $52(65.8)$ & $28(70.0)$ & $24(61.5)$ & 0.016 & .561 \\
\hline $\begin{array}{l}\% \text { feedings breast milk in past week, } \\
\text { Mean (SE) }\end{array}$ & $\begin{array}{l}77.10 \\
(3.95)\end{array}$ & $\begin{array}{l}83.17 \\
(4.49)\end{array}$ & $\begin{array}{l}70.88 \\
(6.44)\end{array}$ & -1.57 & .120 \\
\hline \multicolumn{6}{|l|}{ IGAB Category } \\
\hline Exclusive & $43(53.1)$ & $\begin{array}{c}23 \\
(56.1)\end{array}$ & $20(50.00)$ & & \\
\hline Almost exclusive & - & - & - & & \\
\hline Partial-High, $\geq 80 \%$ & $13(16.1)$ & $8(19.5)$ & $5(12.5)$ & & \\
\hline Partial-Medium, 60\% - 79\% & $6(7.4)$ & $3(7.3)$ & $3(7.5)$ & & \\
\hline Partial-Medium, 40\% - 59\% & $5(6.2)$ & $3(7.3)$ & $2(5.0)$ & & \\
\hline Partial-Medium, 20\% - 39\% & $1(1.2)$ & - & $1(2.5)$ & & \\
\hline Partial-Low, $0 \%-19 \%$ & - & - & - & & \\
\hline Token & $1(1.2)$ & $1(2.4)$ & - & & \\
\hline Not breastfeeding-Never initiated & $2(2.5)$ & - & $2(5.0)$ & & \\
\hline Not breastfeeding-initiated but stopped & $8(9.9)$ & $2(4.9)$ & $6(15.0)$ & & \\
\hline $\begin{array}{l}\text { Estimated baby age at time of } \\
\text { breastfeeding cessation, Mean (SE) }\end{array}$ & $\begin{array}{l}10.31 \\
(0.66)\end{array}$ & $\begin{array}{l}9.69 \\
(0.89)\end{array}$ & $\begin{array}{l}10.88 \\
(0.90)\end{array}$ & 0.93 & .354 \\
\hline
\end{tabular}


Table 12 continued

\begin{tabular}{lccccc}
\hline \multicolumn{1}{c}{ Feeding Characteristic } & Overall & MI & PE & $t / \chi^{2}$ & $P$ \\
& $(N=79)$ & $(n=40)$ & $(n=39)$ & & \\
\hline Perceived behavioral control of & $7.73(0.26)$ & 8.03 & 7.36 & 1.25 & .251 \\
continued breastfeeding, Mean (SE) & & $(0.36)$ & $(0.38)$ & & \\
Importance of continued & $8.42(0.22)$ & 8.28 & 8.54 & -0.58 & .564 \\
breastfeeding, Mean (SE) & & $(0.35)$ & $(0.28)$ & & \\
\hline
\end{tabular}

Note. $\mathrm{MI}=$ Motivational Interviewing; $\mathrm{PE}=$ psychoeducation. Statistical test not conducted for IGAB categories due to small cell sizes.

$* p \leq .05$ 
Considered for Participation, $N=269$

Refused, $N=25$

- $\quad$ Not interested $=11$

- Too busy $=14$

Did not respond to eligibility screener or call to schedule appointment, $N=38$
Not eligible, $N=104$

- Twins $=5$

- Too far away $=20$

- Does not reside in target geographic region $=6$

- Under age $18=2$

- Illicit drug use $=6$

- Too early in pregnancy $=25$

- Too close to due date $=7$

- Multiparous (initial enrollment) $=31$

Scheduled for in-person session, $N=102$

Did not attend, $N=21$

- $\quad$ Cancelled by participant $=14$

- Cancelled by therapist (illness) $=3$

- No-show = 1

- Delivered baby prior to appointment $=3$

Lost to followup, $n=1$
Psychoeducation, $n=40$

Attended in-person session and randomized into groups, $N=81$

Motivational Interviewing, $n=41$

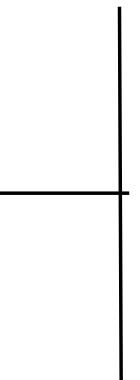




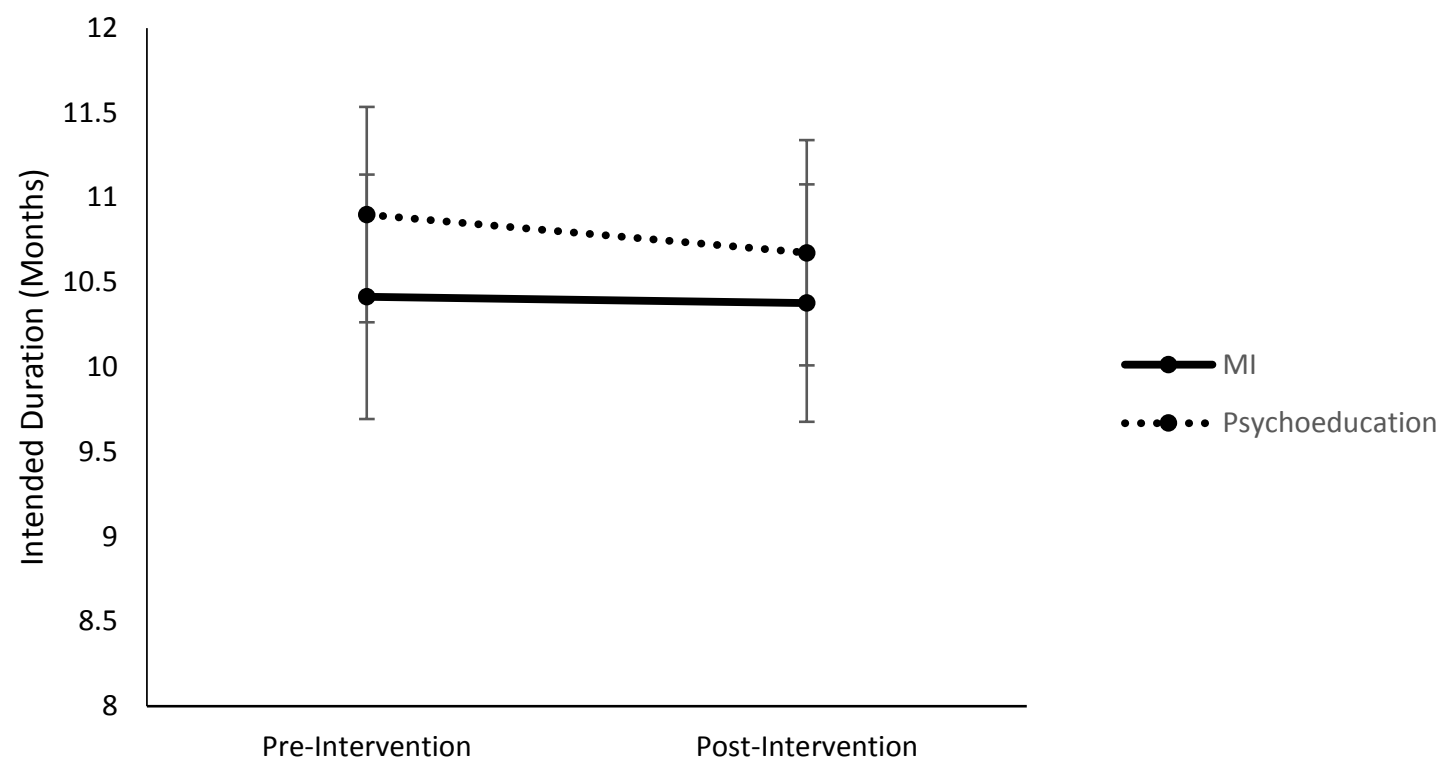

Figure 2. Mean ( \pm 1 SEM) intended breastfeeding duration in months at pre- and postintervention for Motivational Interviewing and psychoeducation groups. 


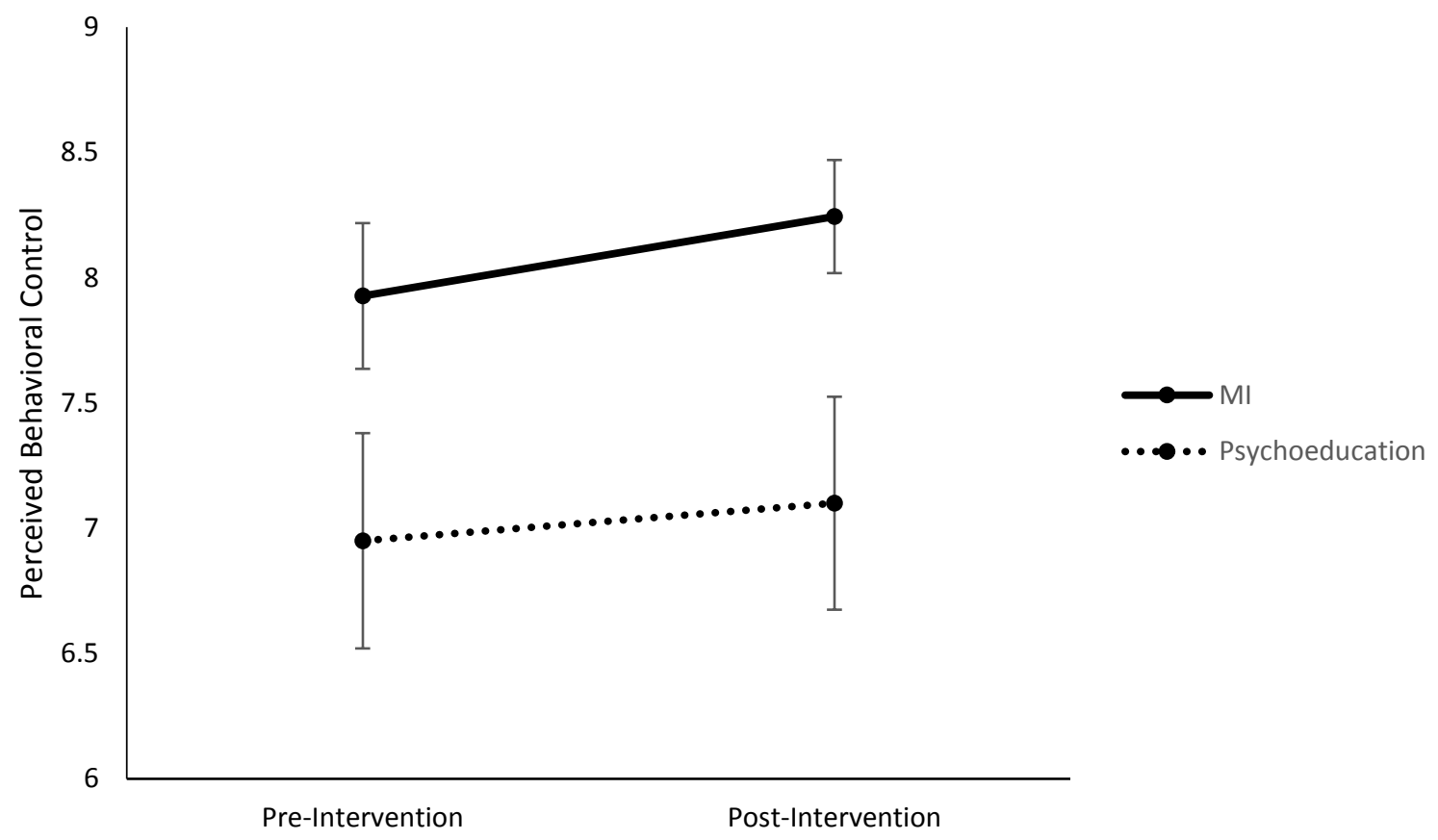

Figure 3. Mean ( \pm 1 SEM) perceived behavioral control of breastfeeding at pre- and postintervention for Motivational Interviewing and psychoeducation groups. 


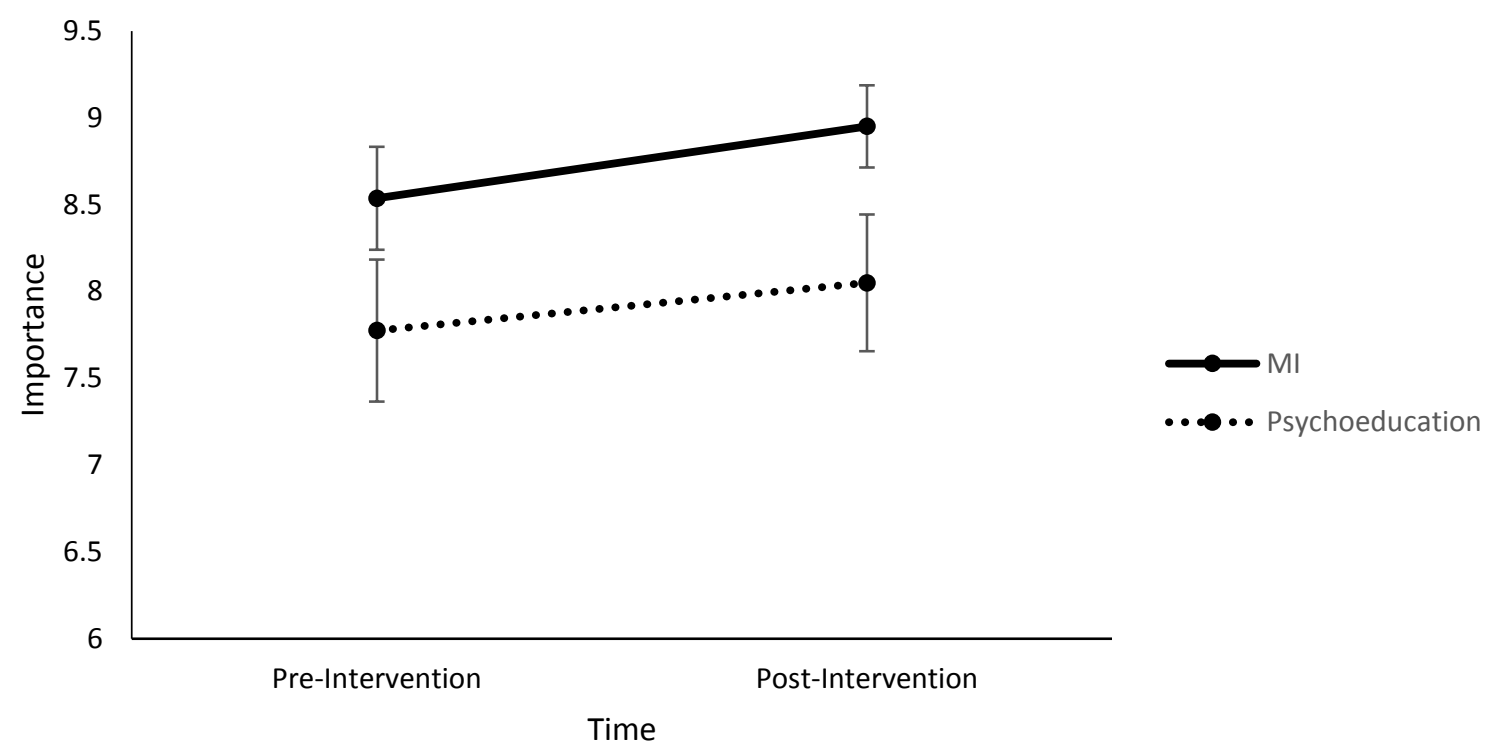

Figure 4. Mean ( \pm 1 SEM) perceived breastfeeding importance at pre- and post-intervention for Motivational Interviewing and psychoeducation groups. 


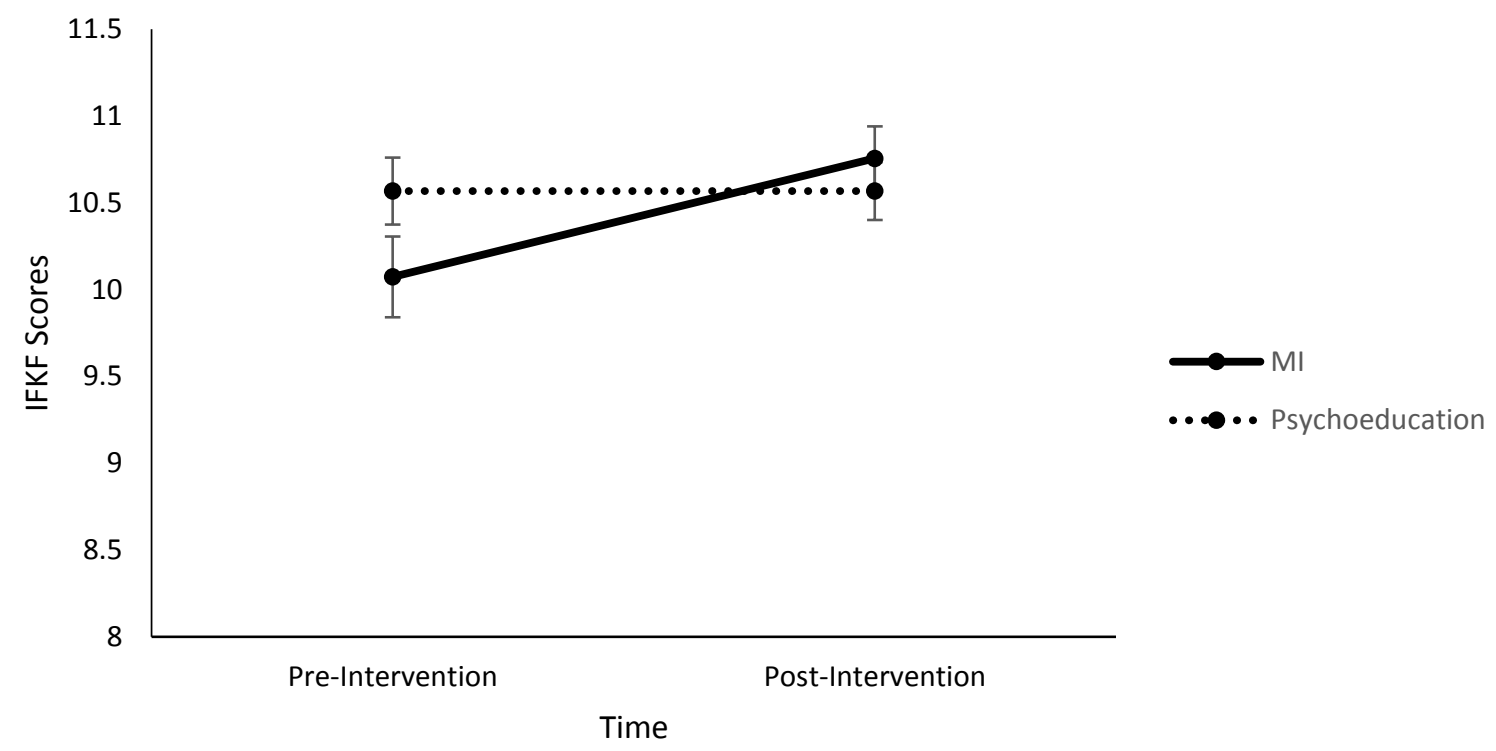

Figure 5. Mean ( \pm 1 SEM) Infant Feeding Knowledge Form (IFKF) scores at pre- and postintervention for Motivational Interviewing and psychoeducation groups. 


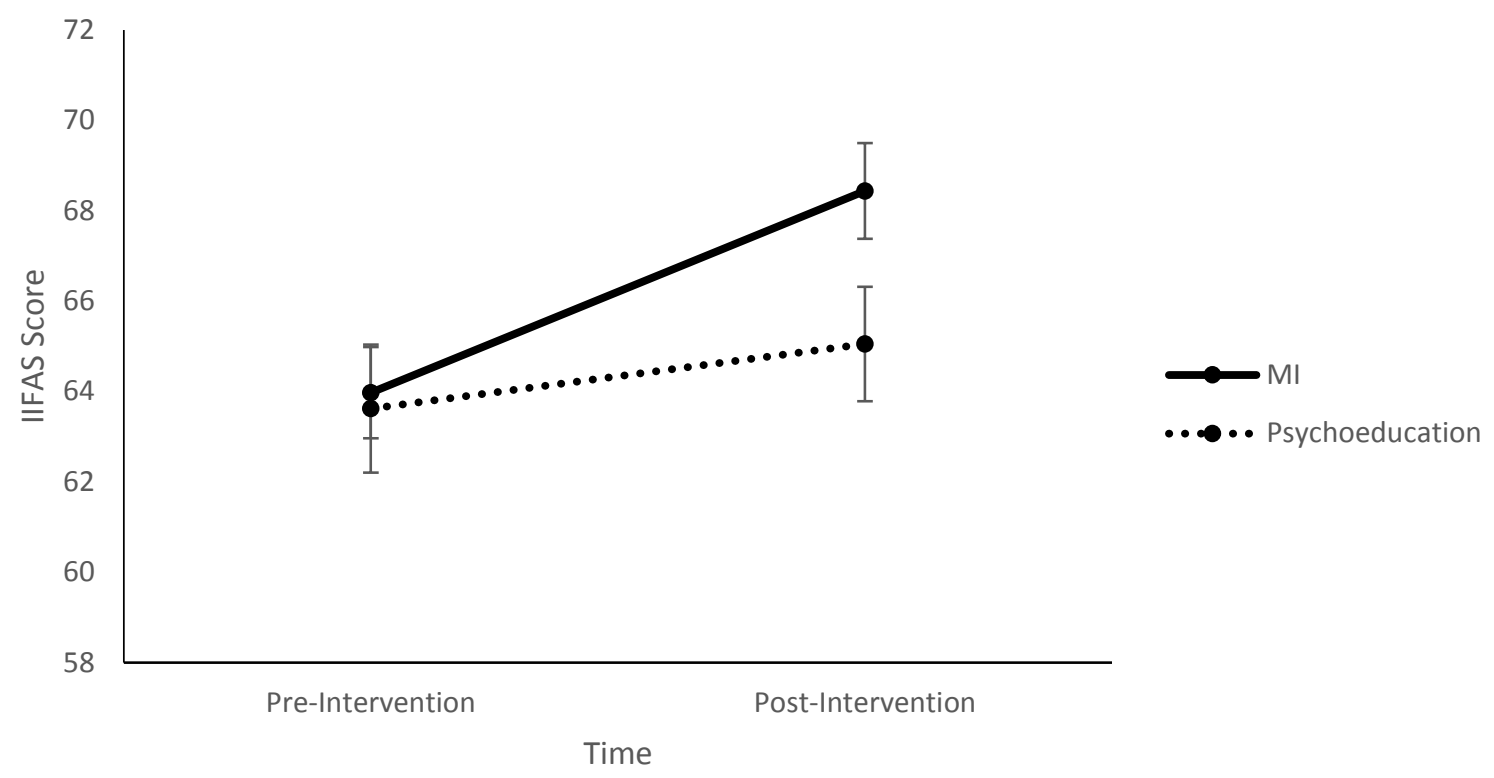

Figure 6. Mean ( \pm 1 SEM) Iowa Infant Feeding Attitudes Scale (IIFAS) scores at pre- and postintervention for Motivational Interviewing and psychoeducation groups. 


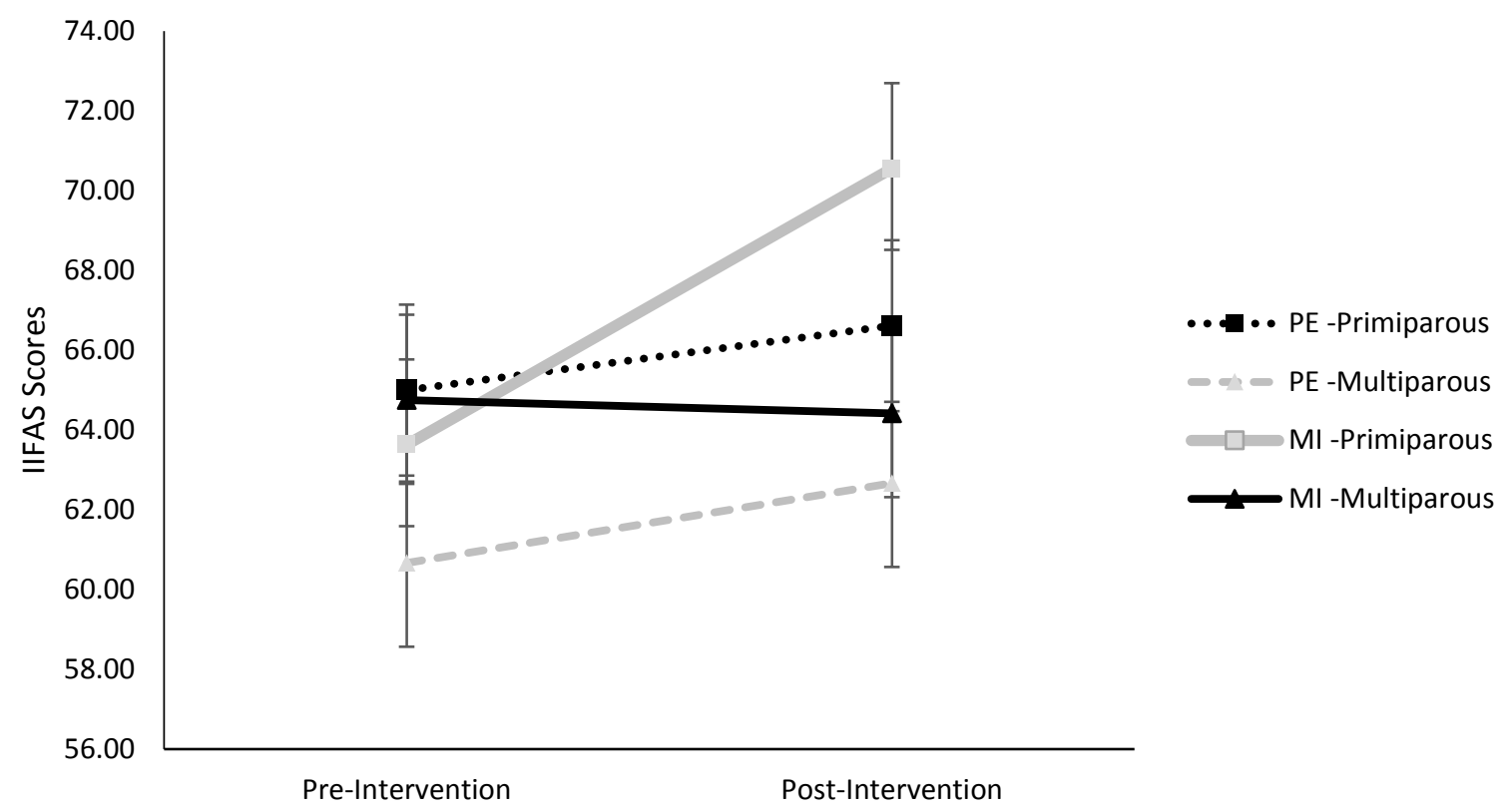

Figure 7. Mean ( \pm 1 SEM) Iowa Infant Feeding Attitudes Scale (IIFAS) scores at pre- and postintervention for Motivational Interviewing and psychoeducation groups by parity status. $\mathrm{MI}=$ Motivational Interviewing; PE = psychoeducation. 


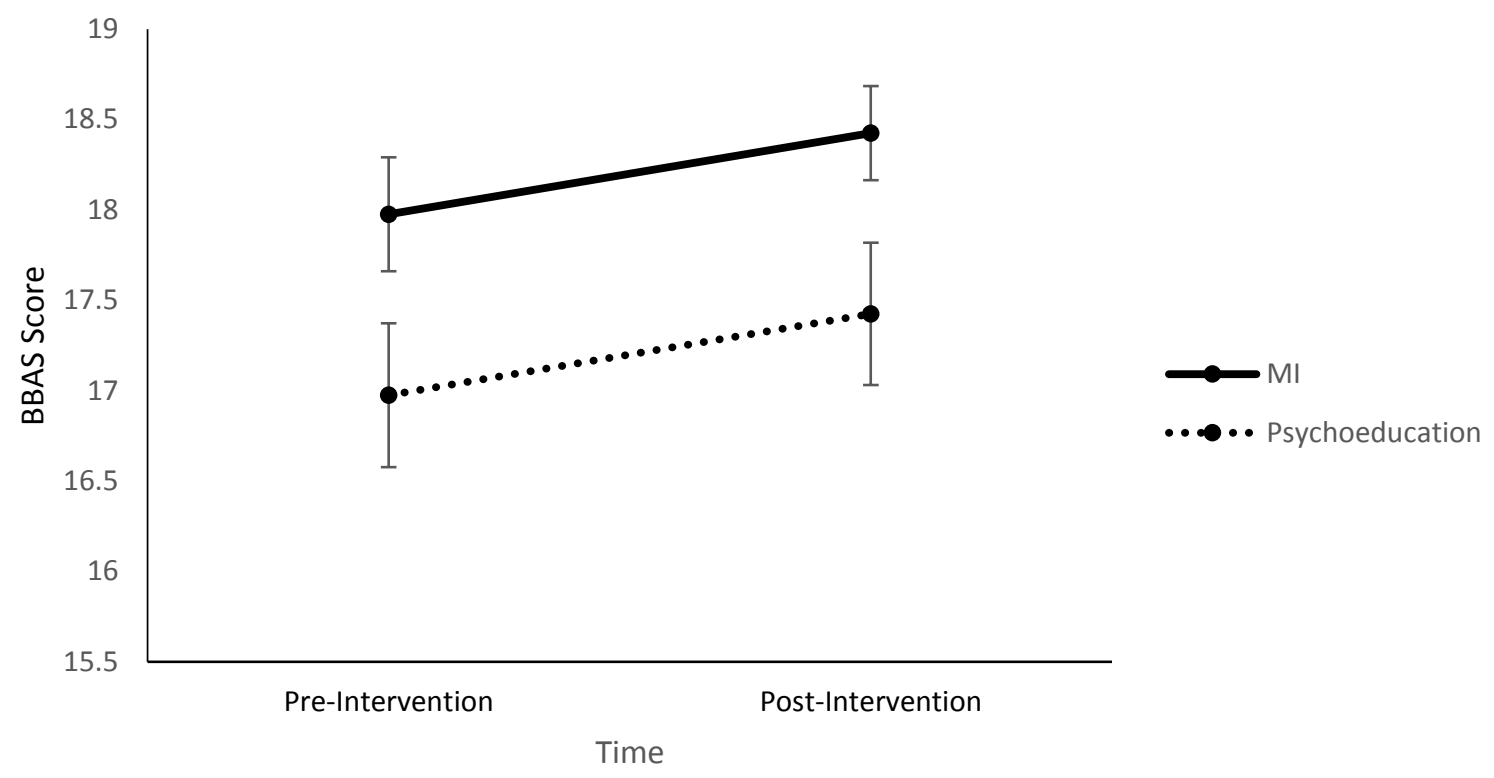

Figure 8. Mean ( \pm 1 SEM) Brief Breastfeeding Attitudes Scale (BBAS) scores at pre- and postintervention for Motivational Interviewing and psychoeducation groups. 


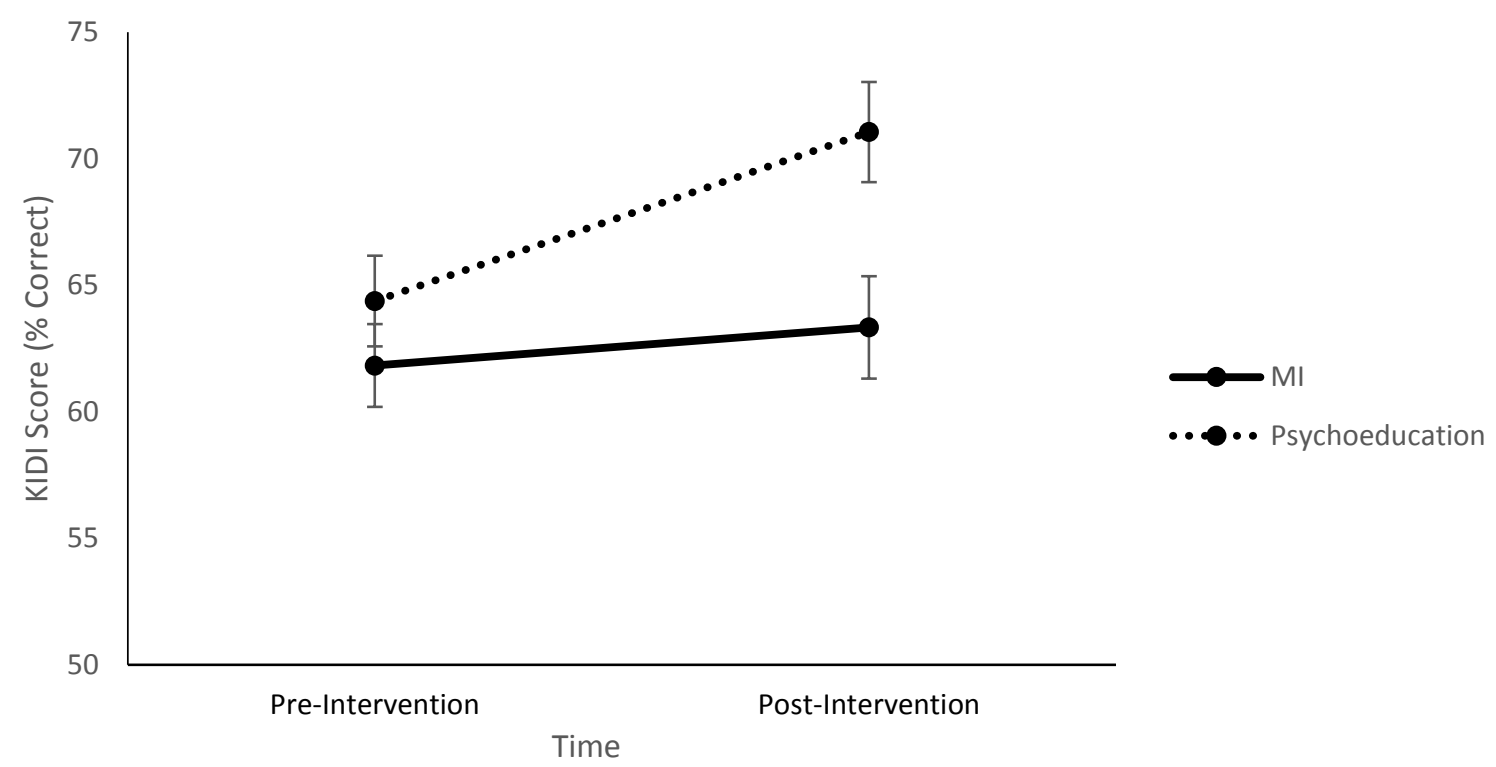

Figure 9. Mean ( \pm 1 SEM) Knowledge of Infant Development Inventory (KIDI) scores at preand post-intervention for Motivational Interviewing and psychoeducation groups. 


\section{Appendix A}

\section{Exclusion and Inclusion Criteria}

\section{Exclusion Criteria}

1. Multiple pregnancy (i.e., twins, triplets, etc.)

2. Has any of the following conditions

○ Developmental or intellectual disability

○ Schizophrenia

○ Any infectious disease (e.g., tuberculosis, Human immunodeficiency virus (HIV)/Acquired immunodeficiency syndrome (AIDS), Hepatitis C)

- Human T-cell lymphotropic virus type I or type II

○ History of lumpectomy or radiation to breast

- Any other known condition of participant or fetus (e.g., congenital organ deformity) that may hinder breastfeeding

3. Actively uses or is dependent upon any of the following substances (Moretti, Lee, \& Ito, 2000):
$\circ$ Heroin
- Cocaine
○ Methamphetamines
- Marijuana
○ Phencyclidine (PCP)
○ Non-prescription opioids (e.g., morphine, oxycodone, hydrocodone)

4. Undergoing any of the following medications/treatments (American Academy of Pediatrics, 2001): 
- Antiretrovirals

○ Cancer chemotherapy (e.g., antimetabolites)

○ Radiation therapy

- Acebutolol

○ Atenolol

○ Bromocriptine

○ Aspirin (salicylates)

- Ergotamine

○ Lithium

○ Phenobarbital

- Primidone

○ Sulfasalazine (salicylazosulfapyridine)

\section{Inclusion Criteria}

1. Age 18 years or older

2. Female

3. Pregnant

4. At least 28 weeks pregnant

5. Able to read, write, speak, and understand English

6. Has access to a phone (either mobile or landline)

7. Lives within driving distance of Morgantown, WV, or is willing to travel to a location that is within driving distance to Morgantown, WV 
Appendix B

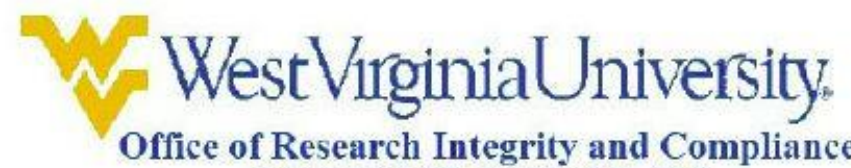

RA6 CHESNUT RIDESROAD MORGANTOWN, WTV 26596

\title{
Approval Letter Expedited
}

\author{
Action Date \\ 07/06/2017 \\ To \\ Daniel McNeil \\ From \\ WVU Office of Research Integrity and Compliance \\ Approval Date \\ $08 / 15 / 2016$ \\ Expiration Date \\ 07/05/2018 \\ Subject \\ Protocol Approval Letter \\ Protocol Number \\ 1605123038R001 \\ Title \\ Effects of prenatal motivational interviewing on infant feeding
}

The above-referenced research study was reviewed by the West Virginia University Institutional Review Board IRB and was approved in accordance with 46 CFR $46.101 \mathrm{~b}$

It has been determined that this study is of minimal risk and meets the criteria as defined by the expedited categories listed below:

- Category 7. Research on individual or group characteristics or behavior (including, but not limited to, research on perception, cognition, motivation, identity, language, communication, cultural beliefs or practices, and social behavior) or research employing survey, interview, oral history, focus group, program evaluation, human factors evaluation, or quality assurance methodologies. [NOTE: Some research in this category may be exempt from the DHHS regulations for the protection of human subjects. See Exempt Categories and 45 CFR 46.101(b)(2) and (b)(3). This listing refers only to research that is not exempt.]

- Please submit a Notify IRB Request to Close Enrollment. This action needs to be taken on any protocol, when enrollment is completed. The protocol can always be reopened to enrollment at a later date. Follow these directions to submit a Request to Close Enrollment: (1) Open the protocol, in 'edit' mode, and go to the Protocol Actions page. In the Available Actions list, select the Request to Close Enrollment action. (2) In the 'Reason' field, please enter the total number of subjects enrolled and the reason for closing enrollment. (3) Attach any relevant information, such as a memo, in the Attachment section of this action. (4) Click the submit button to send the request to the IRB. Upon submission, the KC system will send a notification to the investigator(s) whenever the Request to Close Enrollment action was submitted to the WVU IRB. 


\section{Appendix C}

\section{Prenatal Interview}

This interview consists of a series of questions about you, your family, your health, and your pregnancy. This interview will take about 30 minutes to complete.

\section{DEMOGRAPHICS}

1. Are you or the baby's father of Hispanic, Latino, or Spanish descent?

You, the baby's mother:

$\square$ No, not of Hispanic, Latino, or Spanish descent

$\square$ Yes, Mexican, Mexican American, Chicano

$\square$ Yes, Puerto Rican

$\square$ Yes, Cuban

Yes, other:

The baby's father:

$\square$ No, not of Hispanic, Latino, or Spanish descent

$\square$ Yes, Mexican, Mexican American, Chicano

$\square$ Yes, Puerto Rican

$\square$ Yes, Cuban

$\square$ Yes, other:

2. What is your and the baby's father's race? MARK ALL THAT APPLY.

You, the baby's mother:

White/Caucasian

Black/African American

Hispanic

Asian 
Native American

Other:

The baby's father:

$\square$ White/Caucasian

Black/African American

Hispanic

Asian

Native American

Other:

3. What is your marital status?
$\square$ Single
$\square$ Separated
Divorced
Widowed
Married
$\square$ Live-in partner
$\square$ Other

4. How many years of education do you have?

YEARS

(For example, High School Diploma $=12$ years, College Degree $=16$ years $)$

5. How many years of education does the baby's father have? YEARS

$($ For example, High School Diploma $=12$ years, College Degree $=16$ years $)$

6. What is your yearly household income? That is, how much money does your entire family earn each year? \$ 
7. Ok, so you're saying that your yearly household income is , so it is in the range of [insert appropriate range from those listed below]. Is that correct? IF YES, SELECT APPROPRIATE RANGE. IF NO, GO BACK TO QUESTION 6 TO CLARIFY.

$\square$ Less than $\$ 10,000$

$\$ 10,000$ to $\$ 14,999$

$\$ 15,000$ to $\$ 24,999$

$\$ 25,000$ to $\$ 34,999$

$\$ 35,000$ to $\$ 49,999$

$\$ 50,000$ to $\$ 74,999$

$\$ 75,000$ to $\$ 99,999$

$\square \$ 100,000$ or more

\section{HeAlth AND HeAlth CARE}

8. When is your baby due? Month:

Day:

9. Who provides your prenatal care: MARK ALL THAT APPLY.

An obstetrician

A family doctor, general practitioner, internist, or other physician

A midwife or nurse midwife

Another type of health care provider

I am not getting prenatal care from a health professional $\rightarrow$ GO TO QUESTION 11 .

10. How many weeks pregnant were you when you went for your first prenatal visit?

4 weeks or less

$\square 5$ to 8 weeks 
9 to 12 weeks

13 to 18 weeks

19 to 24 weeks

$\square 25$ weeks or more

11. Are you covered by any kind of health insurance or any kind of health care plan, such as insurance obtained through an employer or a government program like Medicaid?

Yes

\section{No $\rightarrow$ GO TO QUESTION 13}

12. What kind of health insurance do you have? (If participant is unsure, prompt her by asking if she knows what it says on her medical card, or if she has her medical card with her).

$\square$ West Virginia Medicaid

$\square$ Other state's Medicaid

Private

Medicare

13. In the past month, were you enrolled in the WIC program or did you get WIC food or vouchers for yourself or for any of your family? WIC is a program that gives food to pregnant and nursing women, babies, and young children. MARK ALL THAT APPLY.

Yes, I was enrolled or got WIC food for myself

Yes, my child was enrolled or got WIC food

No

14. What was your weight just before you got pregnant?

POUNDS

15. How tall are you? FEET and INCHES

16. What is your age? YEARS 
17. On the average, how many cigarettes do you smoke a day now?

WRITE “O” IF PARTICIPANT DENIES SMOKING CIGARETTES PER DAY

18. How many people not including yourself smoke inside your home most days? Include family members, friends, and anyone else.

$\square \quad 0$
$\square \quad 1$
$\square \quad 2$
$\square \quad 3$
$\square \quad 4$ or more

19. Have you had gestational diabetes with this pregnancy?

$\square$ Yes

$\square$ No

$\square$ Don't know

20. Do you currently have high blood pressure?

$\square$ Yes

$\square \quad$ No

$\square$ Don't know

21. Any other pregnancy complications?

$\square$ Yes, Explain

$\square$ No

\section{EMPLOYMENT}

22. Did you work for pay at any time from the 3 months before you became pregnant up to the present time? 


\section{1] Yes}

$\square \quad$ No $\rightarrow$ GO TO QUESTION 28

23. About how much of your family's income comes from the money you earn from work? If you are no longer working, answer for the time you were working. If you have reduced your work hours because of your pregnancy, answer for the time before you reduced your hours.

$\square$ Less than half

\section{About half}

More than half

24. Do you work for pay now?

Yes, the same number of hours as before pregnancy

$\square$ Yes, but with reduced hours

$\square$ Yes, but on leave until after the baby's birth $\rightarrow$ GO TO QUESTION 26

No $\rightarrow$ GO TO QUESTION 28

25. How many hours per week do you usually work at this job now? If you work at two or more jobs, answer for the total number of hours you work.

1 to 9 hours per week

10 to 19 hours per week

20 to 29 hours per week

30 to 34 hours per week

35 to 40 hours per week

More than 40 hours per week

26. Thinking of work leave that you can use for maternity leave, how many weeks are you eligible for if you have no complications? Please tell me the number of weeks of leave you 
are eligible for in each of the following categories. If you have no leave that you can use for maternity leave, just say “none."

WEEKS OF FULLY PAID LEAVE
WEEKS OF PARTIALLY PAID LEAVE
WEEKS OF UNPAID LEAVE

27. In your opinion, how supportive of breastfeeding is your place of employment?

$\square \quad$ Not at all supportive

$\square \quad$ Not too supportive

$\square$ Somewhat supportive

$\square$ Very supportive

28. Do you plan to work for pay during your baby's first year?
Yes
$\square \quad$ No $\rightarrow$ Go TO QUESTION 33

29. How many weeks after the baby is born do you plan to return to work?

$\square$ Fewer than 4 weeks

$\square \quad 4$ to 6 weeks

$\square \quad 7$ to 9 weeks

$\square \quad 10$ to 12 weeks

$\square \quad 13$ to 16 weeks

$\square \quad 17$ to 20 weeks

$\square \quad 21$ to 30 weeks

$\square \quad$ More than 30 weeks 
30. How many hours per week do you plan to work?

1 to 9 hours per week

$\square 10$ to 19 hours per week

20 to 29 hours per week

$\square 30$ to 34 hours per week

35 to 40 hours per week

$\square$ More than 40 hours per week

31. How many hours per week would you prefer to work when you return to work?

$\square \quad 1$ to 9 hours per week

10 to 19 hours per week

20 to 29 hours per week

30 to 34 hours per week

35 to 40 hours per week

$\square$ More than 40 hours per week

$\square \quad$ Would prefer not to work

32. What will you do with your baby while you are working? MARK ALL THAT APPLY.

$\square$ My baby will be cared for by a family member

$\square \quad$ My baby will be cared for by someone not in my family

$\square \quad$ I will keep my baby with me while I work outside my home

$\square \quad$ I will keep my baby with me while I work at home

$\square \quad$ I have not decided yet

33. What is your job or occupation?

34. Was your current pregnancy planned? 


\section{1) Yes}

$\square$ No

35. What method do you plan to use to feed your new baby in the first few weeks?

$\square$ Breastfeed only (baby will not be given formula)

$\square$ Formula feed only $\rightarrow$ GO TO QUESTION 41

$\square$ Both breast and formula feed

$\square$ Don't know yet $\rightarrow$ GO TO QUESTION 38

36. Do you plan to continue breastfeeding after you return to work?
Yes
No

$\square$ Do not plan to work after the baby's birth

37. How old do you think your baby will be when you completely stop breastfeeding? WEEKS OR_ MONTHS $\rightarrow$ GO TO QUESTION 39

38. If you do decide to breastfeed, how old do you think your baby will be when you completely stop breastfeeding? WEEKS OR MONTHS

39. (If you do decide to breastfeed) How important is it to you that you breastfeed until your baby is [age listed in question 37 or 38), on a scale of 0 to 10 , where 0 means not at all important and 10 means extremely important?

40. (If you do decide to breastfeed) On the same scale of 0 to 10 , how confident are you about being able breastfeed until your baby is [age listed in item 37 or 38$]$ ? 
41. How do the following people think your baby should be fed in the first weeks?

\begin{tabular}{|l|l|l|c|c|c|}
\hline & $\begin{array}{c}\text { Only } \\
\text { breastfeed }\end{array}$ & $\begin{array}{c}\text { Only } \\
\text { formula } \\
\text { fed }\end{array}$ & $\begin{array}{c}\text { Both } \\
\text { breast and } \\
\text { formula } \\
\text { fed }\end{array}$ & $\begin{array}{c}\text { No } \\
\text { opinion or } \\
\text { don't } \\
\text { know }\end{array}$ & $\begin{array}{c}\text { No one in } \\
\text { this } \\
\text { category }\end{array}$ \\
\hline Baby's father & & & & & \\
\hline Your mother & & & & & \\
\hline $\begin{array}{l}\text { Your baby's paternal } \\
\text { grandmother }\end{array}$ & & & & & \\
\hline Your obstetrician or other doctor & & & & \\
\hline
\end{tabular}

Do you have anything you want to add about any of these people?

42. How important are the following people's opinions in your decision about how to feed your baby?

\begin{tabular}{|l|l|l|l|l|l|}
\hline & $\begin{array}{c}\text { Not at all } \\
\text { important }\end{array}$ & $\begin{array}{c}\text { Not very } \\
\text { important }\end{array}$ & $\begin{array}{c}\text { Somewhat } \\
\text { important }\end{array}$ & $\begin{array}{c}\text { Very } \\
\text { important }\end{array}$ & $\begin{array}{c}\text { No one in } \\
\text { this } \\
\text { category }\end{array}$ \\
\hline Baby's father & & & & & \\
\hline Your mother & & & & & \\
\hline $\begin{array}{l}\text { Your baby's paternal } \\
\text { grandmother }\end{array}$ & & & & & \\
\hline Your obstetrician or other doctor & & & & & \\
\hline
\end{tabular}

Do you have anything you want to add about any of these people and/or their opinions?

43. About how many of your friends and relatives have breast fed their babies?

One or two

Three to five

More than five

$\square$ None have breastfed 
$\square \quad$ None have children $\rightarrow$ Go TO QUESTION 45

$\square$ Don't know

44. About how many of your friends and relatives have never breastfed their infants at all?

$\square \quad$ One or two

$\square \quad$ Three to five

$\square \quad$ More than five

$\square \quad$ None -all with babies have breastfed

$\square$ Don't know

45. When you were babies, were you and the baby's father ever breastfed?

You, the baby's mother:

$\square$ Yes

$\square \quad$ No

$\square$ Don’t know

The baby's father:

Yes

$\square$ No

$\square$ Don't know

46. How many other babies have you had or adopted when younger than 12 months old? Do not include the baby you are expecting.

Other babies had

Babies adopted

IF PARTICIPANT HAS GIVEN BIRTH TO OTHER BABIES OR ADOPTED OTHER BABIES, ALSO ASK ITEMS 59-63 ON P. 12 
47. How many times have you been pregnant, not including your current pregnancy? Pregnancies

48. How many abortions have you had?

Abortions

49. How many miscarriages have you had?

Miscarriages

50. Do you have a plan regarding your birth (i.e., preferences or desires)?

$\square$ Yes, specify:

$\square$ No

51. Are you taking any medications?

$\square$ Yes, specify:

$\square$ No

52. Do you have any current or past medical conditions?

$\square$ Yes, specify:

$\square$ No

53. Have you ever been diagnosed with an anxiety disorder?

$\square$ Yes

$\square$ No

54. Do you have a history of being abused physically, emotionally, or sexually?

$\square$ Yes, specify:

$\square$ No

55. Do you have a history of any other type of trauma?

$\square$ Yes, specify: 
$\square \quad$ No

56. Have you ever been diagnosed with depression?

$\square$ Yes

$\square \quad$ No

57. Have you ever been diagnosed with any other mental disorders?

$\square$ Yes, specify:

$\square \quad$ No

58. Have you ever sought counseling or therapy for an emotional or psychological concern?

$\square$ Yes, specify:

ㄱo

\section{FOR MULTIPARAE WOMEN ONLY}

59. What are the ages of your children (both \#1 and \#2) now?

60. Which of these children did you breastfeed? (e.g., \#1, \#2, etc.)

61. How long did you breastfeed each of them? (i.e., less than 1 month, 1-2 months, 3-4 months, 5-6 months, 7-8 months, 9-10 months, 11-12 months, more than 12 months)?

Birth or adopted Breastfed? How old child when stopped?

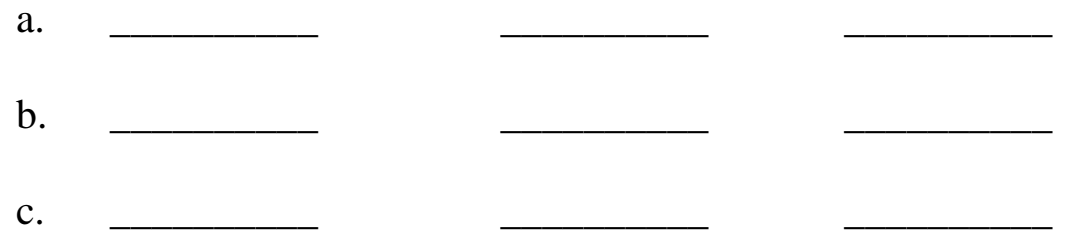

62. Are there any other babies that you breastfed (e.g., a sister's child), and when and how long?

63. How many children (under age 18) live in your home now? 


\section{Appendix D}

Iowa Infant Feeding Attitudes Scale

Instructions: For each of the following statements, please indicate how much you agree or disagree by circling the number that most closely corresponds to your opinion. You may choose any number from 1 to 5 .

\begin{tabular}{|c|c|c|c|c|c|}
\hline & $\begin{array}{l}\text { Strongly } \\
\text { Disagree }\end{array}$ & Disagree & Neutral & Agree & $\begin{array}{c}\text { Strongly } \\
\text { Agree }\end{array}$ \\
\hline $\begin{array}{l}\text { The nutritional benefits } \\
\text { of breast milk last only } \\
\text { until the baby is } \\
\text { weaned from breast } \\
\text { milk. }\end{array}$ & 1 & 2 & 3 & 4 & 5 \\
\hline $\begin{array}{l}\text { 2. Formula-feeding is } \\
\text { more convenient than } \\
\text { breast-feeding. }\end{array}$ & 1 & 2 & 3 & 4 & 5 \\
\hline $\begin{array}{l}\text { 3. Breast-feeding increases } \\
\text { mother-infant bonding. }\end{array}$ & 1 & 2 & 3 & 4 & 5 \\
\hline $\begin{array}{l}\text { 4. Breast milk is lacking in } \\
\text { iron. }\end{array}$ & 1 & 2 & 3 & 4 & 5 \\
\hline $\begin{array}{l}\text { 5. Formula-fed babies are } \\
\text { more likely to be } \\
\text { overfed than are breast- } \\
\text { fed babies. }\end{array}$ & 1 & 2 & 3 & 4 & 5 \\
\hline $\begin{array}{l}\text { 6. Formula-feeding is the } \\
\text { better choice if a } \\
\text { mother plans to work } \\
\text { outside the home. }\end{array}$ & 1 & 2 & 3 & 4 & 5 \\
\hline $\begin{array}{l}\text { 7. Mothers who formula- } \\
\text { feed miss one of the } \\
\text { great joys of } \\
\text { motherhood. }\end{array}$ & 1 & 2 & 3 & 4 & 5 \\
\hline $\begin{array}{l}\text { 8. Women should not } \\
\text { breast-feed in public } \\
\text { places such as } \\
\text { restaurants. }\end{array}$ & 1 & 2 & 3 & 4 & 5 \\
\hline $\begin{array}{l}\text { 9. Babies fed breast milk } \\
\text { are healthier than } \\
\text { babies who are fed } \\
\text { formula. }\end{array}$ & 1 & 2 & 3 & 4 & 5 \\
\hline
\end{tabular}




\begin{tabular}{|c|c|c|c|c|c|}
\hline & $\begin{array}{l}\text { Strongly } \\
\text { Disagree }\end{array}$ & Disagree & Neutral & Agree & $\begin{array}{c}\text { Strongly } \\
\text { Agree }\end{array}$ \\
\hline $\begin{array}{l}\text { 10. Breast-fed babies are } \\
\text { more likely to be } \\
\text { overfed than formula- } \\
\text { fed babies. }\end{array}$ & 1 & 2 & 3 & 4 & 5 \\
\hline $\begin{array}{l}\text { 11. Fathers feel left out if a } \\
\text { mother breast-feeds. }\end{array}$ & 1 & 2 & 3 & 4 & 5 \\
\hline $\begin{array}{l}\text { 12. Breast milk is the ideal } \\
\text { food for babies. }\end{array}$ & 1 & 2 & 3 & 4 & 5 \\
\hline $\begin{array}{l}\text { 13. Breast milk is more } \\
\text { easily digested than } \\
\text { formula. }\end{array}$ & 1 & 2 & 3 & 4 & 5 \\
\hline $\begin{array}{l}\text { 14. Formula is as healthy } \\
\text { for an infant as breast } \\
\text { milk. }\end{array}$ & 1 & 2 & 3 & 4 & 5 \\
\hline $\begin{array}{l}\text { 15. Breast-feeding is more } \\
\text { convenient than } \\
\text { formula-feeding. }\end{array}$ & 1 & 2 & 3 & 4 & 5 \\
\hline $\begin{array}{l}\text { 16. Breast milk is less } \\
\text { expensive than } \\
\text { formula. }\end{array}$ & 1 & 2 & 3 & 4 & 5 \\
\hline $\begin{array}{l}\text { 17. A mother who drinks } \\
\text { alcohol once a week } \\
\text { should not breast-feed } \\
\text { her baby }\end{array}$ & 1 & 2 & 3 & 4 & 5 \\
\hline
\end{tabular}




\section{Appendix E \\ Brief Breastfeeding Attitudes Scale}

Directions: Read each statement carefully and circle the number that best describes your opinion/belief.

For me to feed my baby only breastmilk for the first six months of the baby's life would be:

1. Unpleasant

1
2

1

2. Unenjoyable

1

(1)

2

\section{Inconvenient}

1

2

4. Unhealthy

1
2

3

4

\section{Pleasant}

4

5

\section{Enjoyable}

4

5

\section{Convenient}

5

\section{Healthy}

5 


\section{Appendix F \\ Infant Feeding Knowledge Form}

Instructions: Indicate whether you agree or disagree with the following items.

1. Breastfeeding should be started as soon as possible after the baby is born.
Agree
Disagree

2. Alcohol and caffeine are passed from the mother's body to breast milk.

$$
\text { Agree Disagree }
$$

3. Breastfeeding helps prevent infections and allergies in the baby.

Agree Disagree

4. Most women make enough breast milk to adequately feed the baby.

$$
\text { Agree Disagree }
$$

5. Breastfeeding alone provides sufficient nutrition in the first 6 months of life for the baby.

$$
\text { Agree Disagree }
$$

6. Babies who are formula fed have more illness than babies who are breastfed.

$$
\text { Agree Disagree }
$$

7. Women who have breastfed have lowered risk of breast and ovarian cancer.

$$
\text { Agree Disagree }
$$

8. A woman who has small breasts cannot breastfeed.

$$
\text { Agree Disagree }
$$

9. Breast milk and formula are the same.

$$
\text { Agree Disagree }
$$

10. If breastfeeding, a woman cannot return to work.

$$
\text { Agree Disagree }
$$

11. Breastfeeding prevents a woman from returning to her pre-pregnancy weight.

$$
\text { Agree Disagree }
$$

12. Breastfeeding women should avoid eating certain foods.

$$
\text { Agree Disagree }
$$


Appendix G

Readiness to Change Rulers

Not at all

Extremely

Important

Important

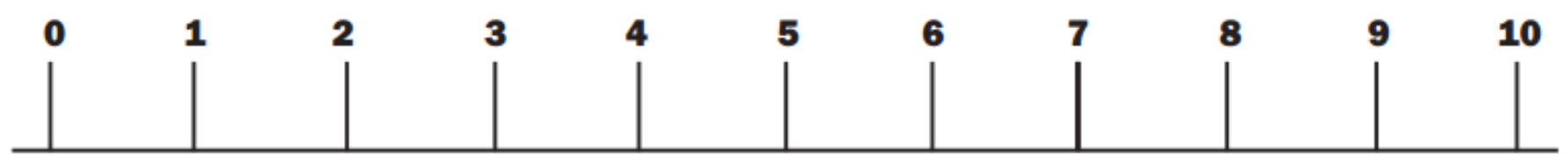

Not at all

Extremely

Confident

Confident

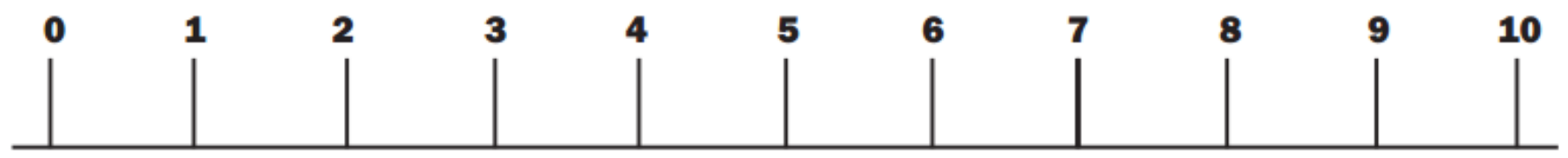




\section{Appendix $\mathrm{H}$}

Post-Intervention Intentions, Importance, and Perceived Behavioral Control

1. What method do you plan to use to feed your new baby in the first few weeks?

Breastfeed only (baby will not be given formula)

Formula feed only $\rightarrow$ STOP. END THIS PORTION OF THE SESSION.

Both breast and formula feed $\rightarrow$

Don't know yet $\rightarrow$ Go TO QUESTION 3

2. How old do you think your baby will be when you completely stop breastfeeding? WEEKS OR MONTHS

3. If you do decide to breastfeed, how old do you think your baby will be when you completely stop breastfeeding?

\section{WEEKS OR _ _ MONTHS}

4. (If you do decide to breastfeed) How important is it to you that you breastfeed until your baby is [age listed in item 2 or 3], on a scale of 0 to 10 , where 0 means not at all important and 10 means extremely important?

5. (If you do decide to breastfeed) On the same scale of 0 to 10 , how confident are you about being able breastfeed until your baby is [age listed in item 2 or 3]?

\section{Sham Questions 6-8 for Psychoeducation Group Only}

6. Name three things you learned today that you did not know before.

7. Would you recommend these handouts to a friend? Why or why not.

8. What are you most looking forward to about the birth of your baby? 


\section{Appendix I}

\section{Postpartum Interview}

Hi! My name is from the WVU Mother and Infant Health Study. How are you doing? Is this a good time to talk? It will take about 15 minutes. (If yes, continue; if no, schedule another time to conduct phone interview). This interview consists of a series of questions about you, your new baby, and you and your new baby's health. Once you finish the interview, I'll confirm your address and send you your gift card in the mail. Do you have any questions? (If yes, answer questions. If no, continue with interview).

1. Is your baby a boy or a girl?

Boy

\section{Girl}

Congratulations!

2. What was your baby's length at birth? INCHES

3. What was your baby's weight at birth? POUNDS and OUNCES

4. What was your baby's APGAR score?

5. Where was your baby delivered?

6. In the past month, were you enrolled in the WIC program or did you get WIC food or vouchers for yourself or for any of your children? WIC is a program that gives food to pregnant and nursing women, babies, and young children. MARK ALL THAT APPLY.

Yes, I was enrolled or got WIC food for myself

Yes, my child was enrolled or got WIC food

No

7. Other than your participation in this study, when you were pregnant with this baby, did you attend any classes or support groups that discussed breastfeeding your baby? 
$\square$ Yes, a class on breastfeeding

Yes, a childbirth or baby care class that included breastfeeding

$\square \quad$ Yes, a support group for breastfeeding

$\square$ No

8. Have you experienced or are you currently experiencing postpartum depression?

- Yes

$\square \quad$ No

$\square \quad$ Not sure

9. Are you taking any medications? If so, please list:

10. Do you work for pay now?

$\square$ Yes, the same number of hours as before I got pregnant

Yes, but with reduced hours

$\square \quad$ Yes, but on maternity leave

No $\rightarrow$ Go TO QUESTION 11

11. How many hours per week do you usually work at this job now? If you work at two or more jobs, answer for the total number of hours you work?

$\square \quad 1$ to 9 hours per week

10 to 19 hours per week

- 20 to 29 hours per week

30 to 34 hours per week

35 to 40 hours per week 
More than 40 hours per week

12. Which type of health professional was your birth attendant?

An obstetrician

A family doctor, general practitioner, internist, or other physician

A midwife or nurse midwife

Another type of health care provider

No health professional was present

13. How was your baby delivered?

Vaginally and not induced

Vaginally and induced

A planned cesarean

An unplanned or emergency cesarean

14. At how many weeks gestation was your baby born? WEEKS

15. Which of the following medications did you have during labor or delivery? MARK ALL THAT APPLY.

General anesthesia (you were put to sleep)

A spinal or epidural

Demerol or Stadol

Nitrous oxide (gas breathed through a mask or mouthpiece while remaining conscious)

Pudendal block or other local blocks (injections into the vagina or cervix before the birth) 
Other pain medication or don't know which pain medication

No pain medication

16. Was your baby admitted to the NICU?

Yes

$\square$ No

If "yes", how many nights did you spend in the NICU?

17. How many nights were you in the hospital or birth center after your baby was born? NIGHTS

18. While you were in the hospital for delivery of this baby, did anyone help you with breastfeeding by showing you how or talking to you about breastfeeding?

Yes

No

If yes, who helped you with breastfeeding? MARK ALL THAT APPLY

Doctor

Nurse

Peer counselor

Lactation consultant (who was it?)

Family member(s)

Friend(s)

Breastfeeding support group member

Someone else (specify)

19. Did you have "skin to skin" contact or "KangarooCare" time with your baby within the first couple hours after he/she was born? 
$\square \quad$ Yes

$\square$ No

$\square$ Don't know/not sure

20. While you were in the hospital or birth center, did your baby stay in your room day and night, except for doctor visits, bathing, or other treatments?

$\square$ Yes, all the time

Yes, some nights but not all

No

21. Was your baby brought to you for feeding during the night?

$\square$ Yes

$\square$ No

22. Does your baby have any serious, long-term medical problems?

$\square$ Yes, specify:

$\square \quad$ No

23. On the average, how many cigarettes do you smoke a day now? WRITE "O" IF PARTICIPANT DENIES SMOKING.

CIGARETTES PER DAY

24. Did your baby receive formula while in the hospital?

$\square$ Yes

$\square$ No

$\square \quad$ Not sure

25. Who is your baby's pediatrician/doctor? 
26. In your opinion, which statement best describes the attitude of the following people about feeding your baby?

\begin{tabular}{|l|l|l|l|l|l|}
\hline & $\begin{array}{c}\text { Favored } \\
\text { breastfeeding } \\
\text { only }\end{array}$ & $\begin{array}{c}\text { Favored } \\
\text { formula } \\
\text { feeding only }\end{array}$ & $\begin{array}{c}\text { Favored } \\
\text { mix } \\
\text { preference for } \\
\text { either method }\end{array}$ & $\begin{array}{c}\text { Don’t } \\
\text { know }\end{array}$ \\
\hline Your doctor & & & & & \\
\hline Baby's doctor & & & & & \\
\hline $\begin{array}{l}\text { Staff of } \\
\text { hospital or } \\
\text { birth center }\end{array}$ & & & & & \\
\hline
\end{tabular}

27. Did you ever breastfeed or try to breastfeed your baby, either in the hospital or birth center, or after you went home?

$$
\begin{aligned}
& \text { Yes } \\
& \text { No ( } \rightarrow \text { GO TO SECTION “NON-BREASTFEEDERS”) }
\end{aligned}
$$

28. When you left the hospital or birth center, how were you feeding your baby?

Breastfeeding only

Formula feeding only

Both breast and formula feeding

29. Using 1 to mean "Disliked Very Much" and 5 to mean "Liked Very Much," how would you say you felt about breastfeeding during the first week you were breastfeeding?

\begin{tabular}{|c|c|c|c|c|}
\hline 1 & & & 5 \\
Disliked & 2 & 3 & 4 & Liked \\
Very & & & Very \\
Much & & & & Much \\
\hline
\end{tabular}


30. Did you have any of the following problems breastfeeding your baby during your first 2 weeks of breastfeeding? MARK ALL THAT APPLY

My baby had trouble sucking or latching on

I didn't have enough milk

My baby choked

My nipples were sore, cracked, or bleeding

My baby wouldn't wake up to nurse regularly enough

My breasts were overfull (engorged)

My baby was not interested in nursing

I had a yeast infection of the breast

My baby got distracted

I had a clogged milk duct

My baby nursed too often

$\square$ My breasts were infected or abscessed

It took too long for my milk to come in

My breasts leaked too much

I had trouble getting the milk flow to start

$\square \quad$ I had some other problem

My baby didn't gain enough weight or lost too much weight

I had no problems

31. Have you completely stopped breastfeeding and pumping milk for your baby?

Yes $\rightarrow$ Go TO SECTION “INITIATED, BUT NOT CURRENTLY BREASTFEEDING AT 1 MONTH POSTPARTUM") 
$\square \quad$ No

32. In the past 7 days, how often was your baby fed each food listed below? Include feedings by everyone who feeds the baby and include snacks and night-time feedings.

\begin{tabular}{|l|l|l|}
\hline & $\begin{array}{c}\text { Feedings Per } \\
\text { Day }\end{array}$ & $\begin{array}{c}\text { Feedings Per } \\
\text { Week }\end{array}$ \\
\hline Breast milk & & \\
\hline Formula & & \\
\hline Other (PLEASE SPECIFY) & & \\
\hline
\end{tabular}

33. Overall, about what percentage of your baby's feedings are breastmilk?
$0-20 \%$
$20-40 \%$
- 40-60\%
$60-80 \%$
$\square \quad 80-100 \%$

34. (If Fed Formula): How old was your baby when he or she was first fed formula?

$\square \quad 1$ day or less

$\square \quad 2$ to 6 days

$\square \quad 7$ to 13 days

$\square \quad 14$ to 20 days

$\square$ More than 20 days

$\square \quad$ Never fed formula 
35. Which of the following was your baby given in vitamin or mineral drops at least 3 days a week during the past 2 weeks? If your baby was given drops that contained more than one of the items listed, please mark each of the separate items. MARK ALL THAT APPLY.

Fluoride

\section{Vitamin D}

Iron

\section{Other vitamins}

None of these

36. About how long does an average breastfeeding last?

Less than 10 minutes

10 to 19 minutes

20 to 29 minutes

30 to 39 minutes

40 to 49 minutes

50 or more minutes

37. In an average 24-hour period, what is the LONGEST time for you, the mother, between breastfeedings or expressing milk? Please count the time from the start of one breastfeeding or expressing session to the start of the next. Please think of time between feedings during both night and day to find the longest time.

HOURS AND MINUTES

38. How many times in the past 7 days was your baby fed expressed or pumped breast milk to drink? (Write in 0 if your baby was not fed expressed or pumped milk to drink.) TIMES 
39. How old do you think your baby will be when you completely stop breastfeeding? MONTHS

40. How important is it to you that you breastfeed until your baby is [age listed in prior item], on a scale of 0 to 10 , where 0 means not at all important and 10 means extremely important?

41. On the same scale of 0 to 10 , how confident are you about being able breastfeed until your baby is [age listed in prior item]?

INITIATED, BUT NOT CURRENTLY BREASTFEEDING AT ONE MONTH POSTPARTUM

42. Did you breastfeed as long as you wanted to?

$$
\text { Yes }
$$

$$
\text { No }
$$

43. How old was your baby when you completely stopped breastfeeding and pumping milk? DAYS (if younger than 2 weeks) OR WEEKS

44. What were your reasons behind your decision to stop breastfeeding your baby? MARK ALL THAT APPLY.

My baby had trouble sucking or latching on

My baby became sick and could not breastfeed

My baby began to bite

My baby lost interest in nursing or began to wean him or herself

My baby was old enough that the difference between breast milk and formula no longer mattered

Breast milk alone did not satisfy my baby 
I thought that my baby was not gaining enough weight

A health professional said my baby was not gaining enough weight

I had trouble getting the milk flow to start

I didn't have enough milk

My nipples were sore, cracked, or bleeding

My breasts were overfull or engorged

My breasts were infected or abscessed

My breasts leaked too much

Breastfeeding was too painful

Breastfeeding was too tiring

I was sick or had to take medicine

Breastfeeding was too inconvenient

I did not like breastfeeding

I wanted to be able to leave my baby for several hours at a time

I wanted to go on a weight loss diet

I wanted to go back to my usual diet

I wanted to smoke again or more than I did while breastfeeding

I had too many household duties

I could not or did not want to pump or breastfeed at work

Pumping milk no longer seemed worth the effort that it required

I was not present to feed my baby for reasons other than work

I wanted or needed someone else to feed my baby

Someone else wanted to feed the baby 
I did not want to breastfeed in public

I wanted my body back to myself

I became pregnant or wanted to become pregnant again

45. In the past 7 days, how often was your baby fed each food listed below? Include feedings by everyone who feeds the baby and include snacks and night-time feedings.

\begin{tabular}{|l|l|l|}
\hline & $\begin{array}{c}\text { Feedings Per } \\
\text { Day }\end{array}$ & $\begin{array}{c}\text { Feedings Per } \\
\text { Week }\end{array}$ \\
\hline Breast milk & & \\
\hline Formula & & \\
\hline Other (PLEASE SPECIFY) & & \\
\hline
\end{tabular}

\section{NON-BREASTFEEDER SECTION}

46. What were your reasons for your decision not to breastfeed your baby? MARK ALL THAT $A P P L Y$.

My baby was sick and could not breastfeed

I thought I would not have enough milk

A health professional said I should not breastfeed for medical reasons

I was sick or had to take medicine

I believe that formula is as good as breastfeeding or that formula is better

I thought that breastfeeding would be too inconvenient

I tried breastfeeding before and didn't like it or it didn't work out

I wanted to be able to leave the baby for several hours at a time

I wanted to go on a weight loss diet

I wanted to go back to my usual diet 
$\square \quad$ I wanted to smoke again or smoke more than I should while breastfeeding

I had too many household duties

I planned to go back to work or school

I wanted or needed someone else to feed my baby

$\square$ Someone else wanted to feed the baby

$\square \quad$ I wanted my body back to myself

The baby's father didn't want me to breastfeed

The baby's grandmother didn't want me to breastfeed

I wanted to use contraception that can't be used while breastfeeding

47. In the past 7 days, how often was your baby fed each food listed below? Include feedings by everyone who feeds the baby and include snacks and night-time feedings.

\begin{tabular}{|l|l|l|}
\hline & $\begin{array}{c}\text { Feedings } \\
\text { Per Day }\end{array}$ & $\begin{array}{c}\text { Feedings Per } \\
\text { Week }\end{array}$ \\
\hline Breast milk & & \\
\hline Formula & & \\
\hline Other (PLEASE SPECIFY) & & \\
\hline
\end{tabular}




\section{Appendix $\mathbf{J}$}

\section{Participant Eligibility Screener}

1. Are you under the age of 18 ?

2. To the best of your knowledge are you less than 28 weeks pregnant?

3. To the best of your knowledge, are you having a multiple birth (twin or triplets)?

4. Do you have any trouble reading, writing, speaking, or understanding English?

\section{If "YES" to any of these four questions, EXCLUDE.}

Do you have any of the following conditions?

Developmental or intellectual disability

Schizophrenia

Infectious disease, including but not limited to: tuberculosis HIV/AIDS, Hepatitis C

Human T-cell lymphotropic virus type I or type II

History of lumpectomy or radiation to breast

Any other condition that would prevent you from breastfeeding

\section{If "YES" to any of these conditions, EXCLUDE.}

Are you currently using any of the following drugs or medicines?

Heroin

Cocaine

Methamphetamines

Marijuana

Phencyclidine (PCP)

Non-prescription opioids (e.g., morphine, oxycodone, hydrocodone)

Antiretrovirals

Cancer chemotherapy (e.g., antimetabolites)

Radiation therapy

Acebutolol

Atenolol

Bromocriptine

Aspirin (salicylates)

Ergotamine

Lithium

Phenobarbital

Primidone

Sulfasalazine (salicylazosulfapyridine)

If "YES" to any of these drugs or medicines, EXCLUDE. 
Appendix K

Participant Logbook

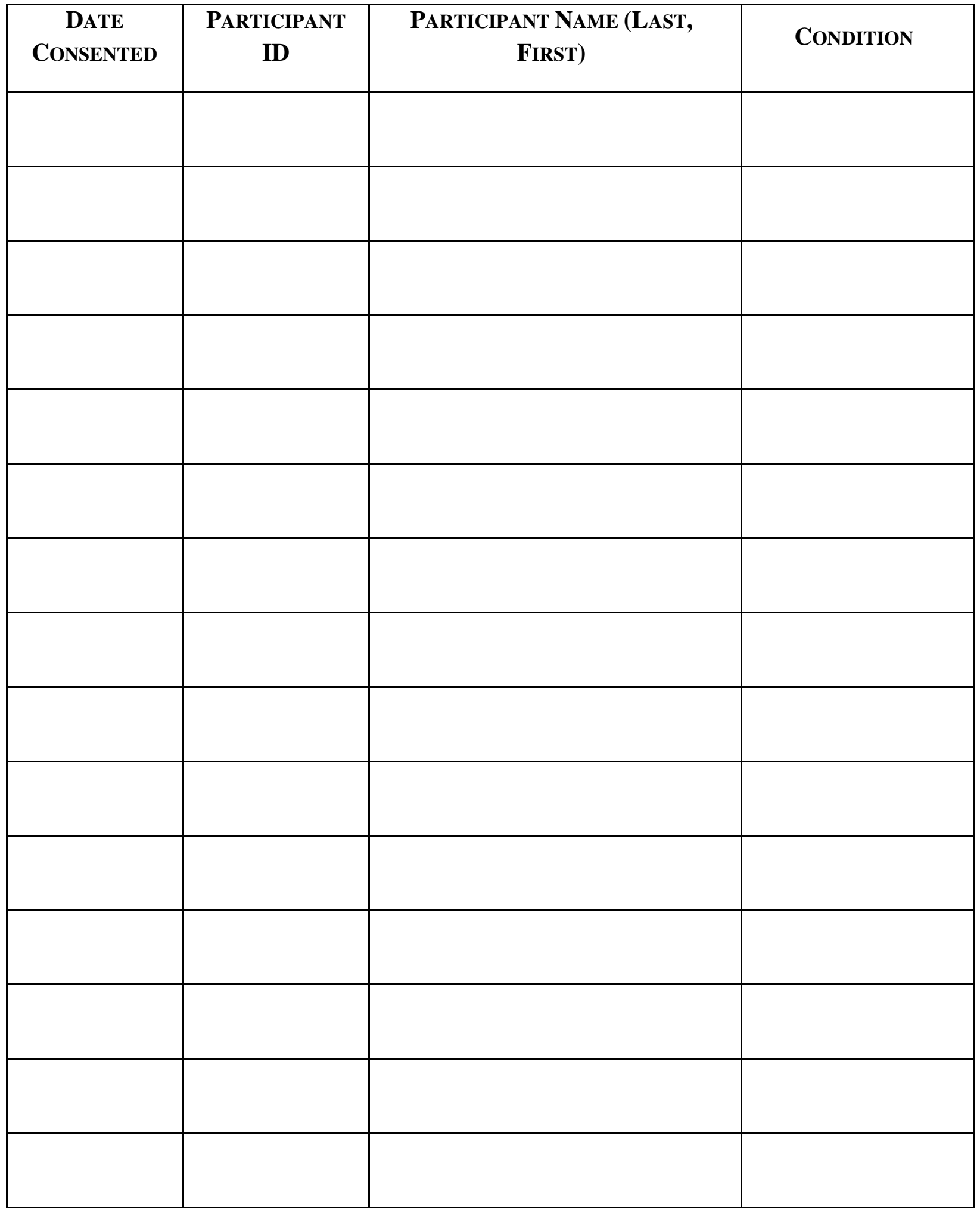


Appendix L

Call Screen and Contact Log

Participant Name:

Participant Phone:

Alternate Phone:

E-mail:

Address:

Mode of Initial Contact: $\square$ E-mail $\quad \square$ Phone $\quad \square$ In-person Contact $\quad \square$ Other

How did you hear about us?

Inclusion Criteria:

Exclusion Criteria:

\section{Contact Log}

\begin{tabular}{|l|l|l|l|l|}
\hline Date & RA & $\begin{array}{c}\text { Mode of } \\
\text { Contact }\end{array}$ & Reason & Notes \\
\hline & & & & \\
\hline & & & & \\
\hline & & & & \\
\hline & & & & \\
\hline & & & & \\
\hline
\end{tabular}

Scheduled:

$\square$ Not Scheduled

Notes: 
Appendix M

Refusal Logbook

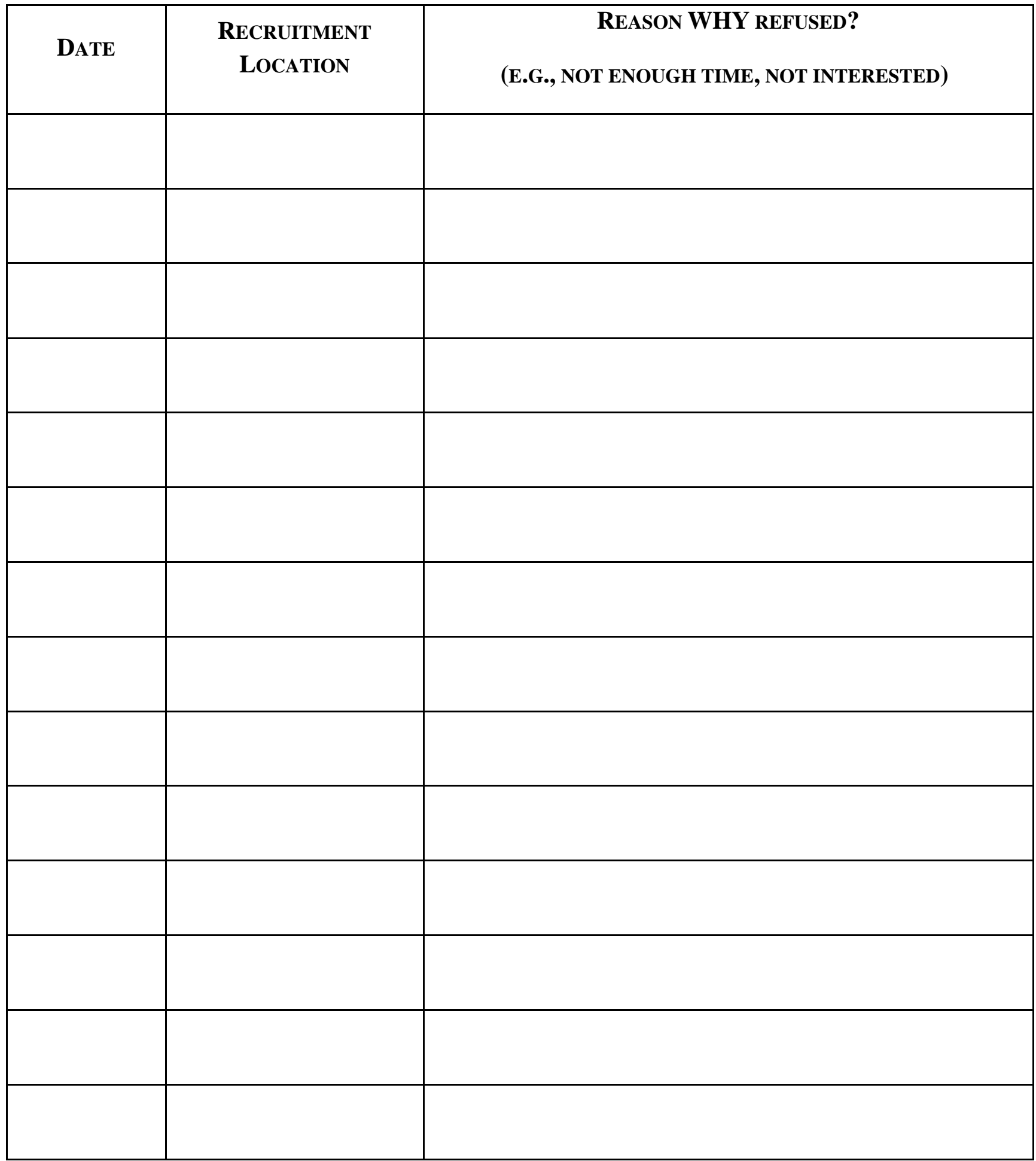




\section{Appendix N}

\section{Certainty Randomization Item}

How certain are you that you will feed your baby only breastmilk for your baby's first six months?

Completely
certain that I
will NOT feed
$\begin{gathered}\text { my baby only } \\ \text { breastmilk for } \\ \text { six months }\end{gathered}$




\section{Appendix O}

Coding Sheets

Name of Coder:

\section{Group}

Date of Coding:

\begin{tabular}{|c|c|c|}
\hline Topic & 口 & $\begin{array}{c}\text { Record time of first occurrence on video } \\
\text { (e.g., 10:00 = ten minutes) }\end{array}$ \\
\hline Breastfeeding initiation & $\square$ & \\
\hline Breastfeeding duration & $\square$ & \\
\hline Breastfeeding exclusivity & $\square$ & \\
\hline Benefits of breastfeeding & $\square$ & \\
\hline Dedication to baby's health & $\square$ & \\
\hline Readiness to Change Ruler-Confidence & $\square$ & \\
\hline Readiness to Change Ruler-Importance & $\square$ & \\
\hline Perceived barriers & $\square$ & \\
\hline
\end{tabular}




\section{Psychoeducation Group}

Name of Coder:

Date of Coding:

\begin{tabular}{|c|c|c|}
\hline Topic & $\nabla$ & $\begin{array}{l}\text { Record time of first occurrence on } \\
\text { video (e.g., 10:00 = ten minutes) }\end{array}$ \\
\hline Define "developmental milestone" & $\square$ & \\
\hline 0-3 months of age developmental issues & $\square$ & \\
\hline AAP Breastfeeding guidelines & $\square$ & \\
\hline Breastfeeding information handout & $\square$ & \\
\hline 3-6 months of age developmental issues & $\square$ & \\
\hline 6-9 months of age developmental issues & $\square$ & \\
\hline 9-12 months of age developmental issues & $\square$ & \\
\hline 12-15 months of age developmental issues & $\square$ & \\
\hline
\end{tabular}




\section{Appendix P \\ Results of "Sham Questions" for Psychoeducation Condition}

Nearly all $(n=38,95.0 \%)$ of the participants in the Psychoeducation Condition answered these questions. Not all participants, however, provided answers for all of the "sham" questions. Therapists did not probe for additional information from participants. Although the participants were asked to name three new things they learned from the Psychoeducation session, only 24 participants $(60.0 \%)$ provided answers for all three new things. As such, the "three new things" questions yielded 93 answers from 38 participants. Participants most commonly stated that they learned specific ages at which babies can perform specific behaviors (e.g., rolling over, sitting up, gripping toys, speaking words, etc.). The next most popular answer was that they had learned about typical patterns of crying, colic, and ways to cope with baby's crying. The third most popular response was citing a fact learned from one of the research studies described in the handouts).

All 38 participants stated that they would recommend the "Zero to Three" handouts to a friend, with top reasons being that the handouts were "informative," "helpful," "straightforward," "organized," and "easy to understand." Several participants said that the handouts would be particularly helpful for first-time parents. Regarding to what the participants were most looking forward to, the top answers, were meeting the baby (e.g., seeing what baby looks like, observing baby's personality), holding/cuddling the baby, and having another child/seeing other child(ren) interact with a new sibling. 
Appendix Q

MI Session Topics "Bubble Sheet"
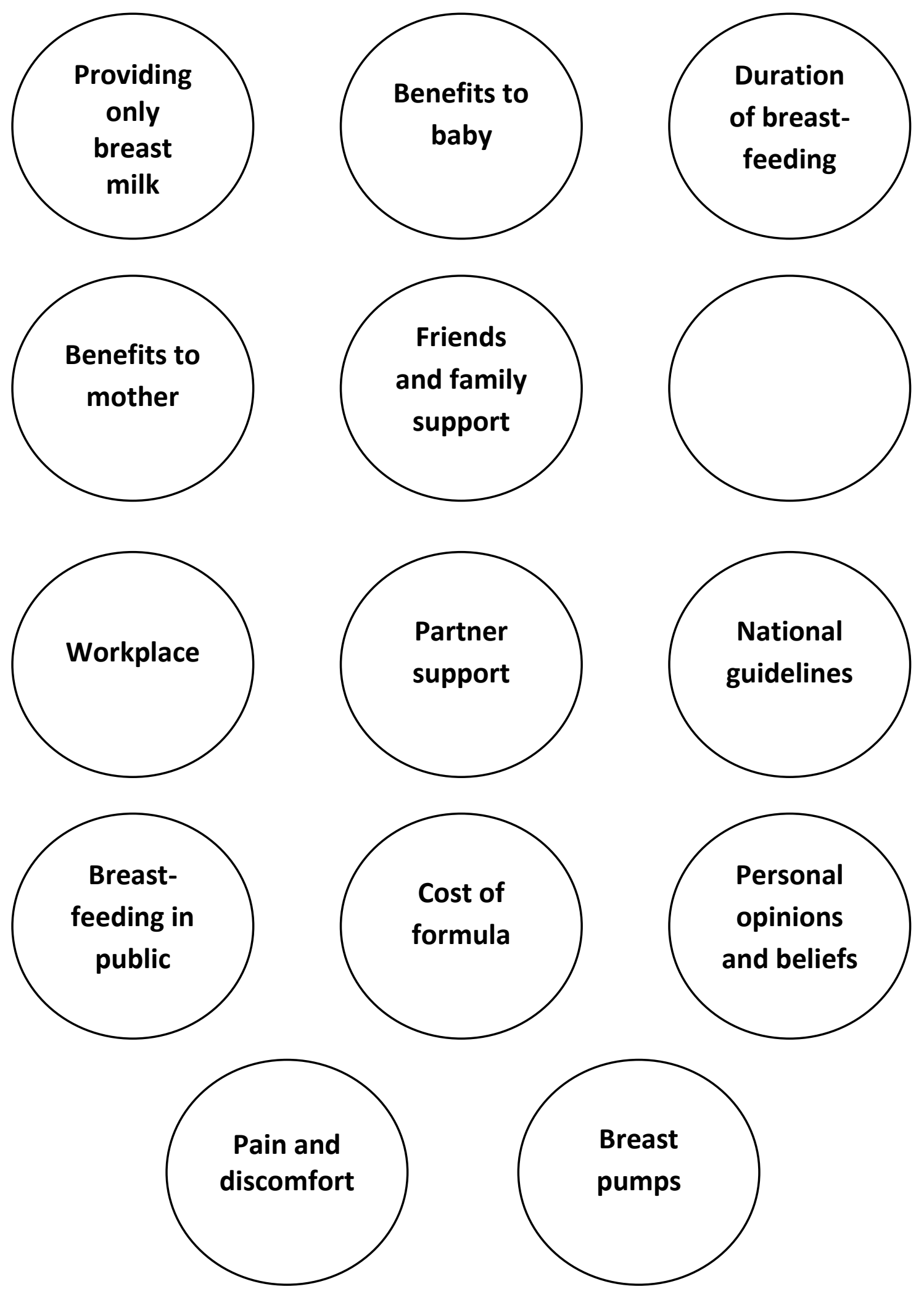


\section{Appendix R}

\section{Group Session Protocol}

\section{Session Duration: $45( \pm 5)$ minutes}

\section{Introductions ( 2 minutes)}

Thank you again for coming to participate in our study today. We appreciate your time and effort. Before we begin, I want to give you an overview of what we will be talking about today. We'll talk about breastfeeding your baby, other methods of feeding your baby, your thoughts on the topic. Today is really all about you and what you want to discuss.

\section{Participant-Centered Conversation ( 40 minutes)}

Although topics of each discussion were unique to each participant, and the total number of topics covered varied between participants, all sessions included the following components/talking points: breastfeeding initiation, breastfeeding duration, breastfeeding exclusivity, benefits of breastfeeding, participant's dedication to baby's health, and perceived barriers. The following figure depicts a basic structure for each session:

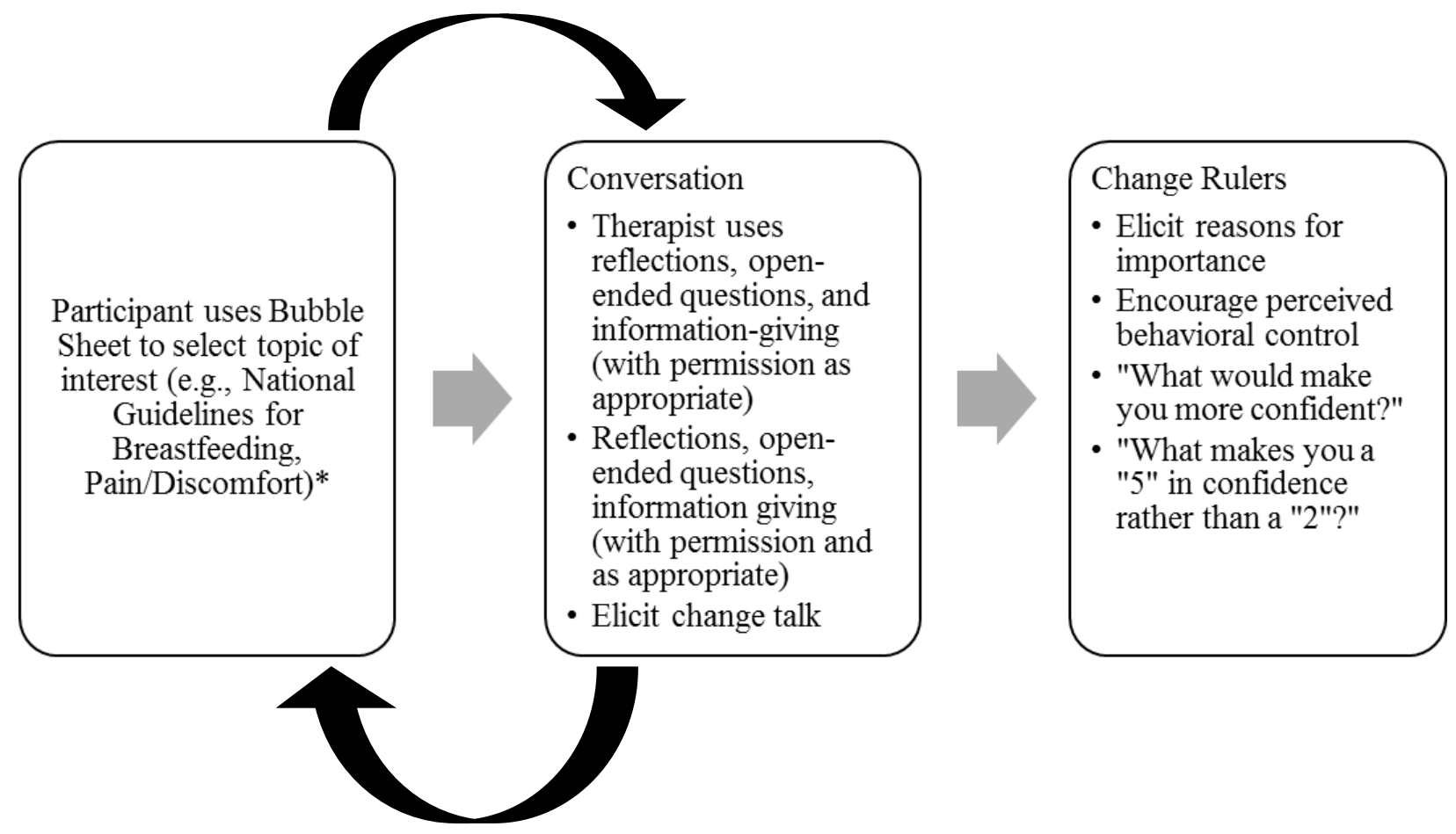




\author{
Appendix $S$ \\ Psychoeducation Group Session Protocol \\ Psychoeducation Session Duration: $45( \pm 5)$ minutes
}

\title{
Introductions ( 2 minutes)
}

Thank you again for coming to participate in our study today. We appreciate your time and effort. Before we begin, I want to give you an overview of what we will be talking about today. We'll talk about different developmental stages that you can expect with your baby. We will also talk briefly about breastfeeding.

\section{Developmental Milestones ( 5 minutes)}

First, let's talk about what developmental milestones. According to the U.S. National Library: "Developmental milestones are behaviors or physical skills seen in infants and children as they grow and develop. Rolling over, crawling, walking, and talking are all considered milestones. The milestones are different for each age range. There is a normal range in which a child may reach each milestone. For example, walking may begin as early as 8 months in some children. Others walk as late as 18 months and it is still considered normal. One of the reasons for wellchild visits to the health care provider in the early years is to follow your child's development. Most parents also watch for different milestones. Talk to your child's provider if you have concerns about your child's development. Closely watching a "checklist" or calendar of developmental milestones may trouble parents if their child is not developing normally. At the same time, milestones can help to identify a child who needs a more detailed check-up. Research has shown that the sooner the developmental services are started, the better the outcome. Examples of developmental services include: speech therapy, physical therapy, and developmental preschool." 


\section{Show handout 1 (0-3 months) and go over it ( 5-7 minutes)}

\section{Birth to 3 Months}

\section{Your Baby's Development}

The first 3 months are all about bables learning to feel comfortable, safe, and secure in the world. By responding to their signals and providing lots of love and comfort, you help them form a trusting bond with you. How are you helping your baby leam to feel safe and secure?

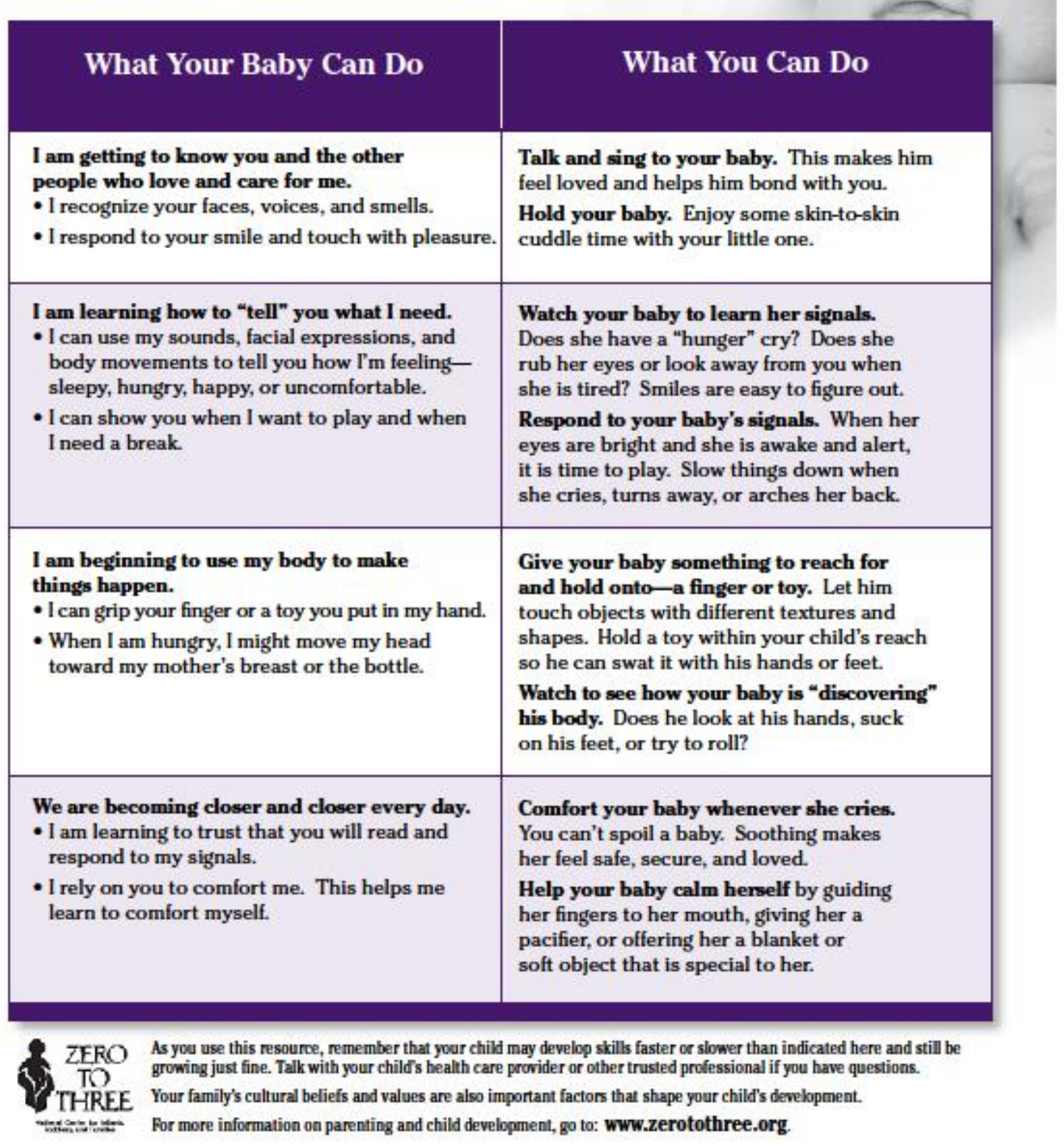




\section{Show handout 2 (early feeding) and go over it ( 5-7 minutes)}

\section{$\longrightarrow \cdots+$ When You Go Home $\downarrow \downarrow \downarrow \downarrow$}

\section{Building your milk supply:}

- Feed early and often, at the earliest signs of hunger.

- 8-12 feedings per 24 hours is expected, although these feedings may not follow a regular schedule.

- Avoid pacifiers or bottles, at least in the first 4 weeks.

- Frequent feeds, not formula Only use formula if there's a medical reason.

- Sleep near your baby, even at home. Learn to nurse lying down.

\section{Feed at the earliest signs of hunger:}

" Hands to mouth, sucking movements.

" Soft cooing, sighing sounds, or stretching

- Crying is a late sign of hunger: don't wait until then!

\section{Watch the baby, not the clock.}

- Alternate which breast you start with, or start with the breast that feels most full.

- Switch sides when swallowing slows or infant takes himself off.

- If's OK if baby doesn't take the second breast at every feed.

- Help baby open his mouth widely. If you're having trouble with latch, get help prompty.

- If the baby is sleqpy: skin-to-skin contact can encourage feeding - Remove baby's top and place him on your bare chest.

\section{Look for signs of milk transfer:}

- You can hear the baby swallowing or gulping.

- There are no dicking or smacking sounds.

- Baby no longer shows signs of hunger after a feed.

- Baby's body and hands are relaxed for a short time.

- You may feel milk let-down:

$\Delta$ You may feel relaxed, drowsy, or thirsty, and you may have tingling in your breasts.

$\Delta$ You may feel some contractions in your uterus, or your other breast may leak milk.

" You should feel strong tugging, but NOT persistent pain. Proper latch prevents pain:

$\Delta$ "chin-to-breast, chest-to-chest"

A "flip lips for a sipx" baby's lips flare outward

$\Delta$ wide open mouthr baby's mouth covers most of the areola (dark area of breast)—not just the nipple.

- Baby has adequate weight gain: follow up 2 days after you get home and again at 2 weeks.

\section{What goes in, must come out. Look for:}

- At least 3 poops per day by day 4 .

- Poops change from dark black to green/brown

to loose yellow as your millk comes in.

- At least 6 heavy/wet diapers after day 4 .

- Urine should be pale yellow as your milk comes in.

\section{Over time:}

- All babies have days when they nurse more frequently.

" Breast swelling normally lessens at about 7-10 days and it is NOT a sign of decreased milk supply.

- Your milk may look thin or bluish, but it contains plenty of nutrients.

\section{If you choose to share a bed with your baby:}

- Keep the bed away from walls on both sides so the baby won't get stuck.

" Avoid heavy blankets, comforters, or pillows.

" Avoid soft surfaces such as waterbeds, couches, and daybeds.

- Neither parent should be under the influence of alcohol, illegal drugs, or medications that would affect the ability to wake up.

- As with sleeping separately put the baby to sleep on his back.

" Do not allow the baby to sleep alone on an adult bed.

" Do not allow anyone except the baby's parents to share a bed with the baby.

- Because the risk of Sudden Infant Death Syndrome is higher in children of smokers, parents who smoke should not bedshare, but may sleep with the baby nearby.

\section{Tell your hospital what you think:}

" Let your hospital know if you had a good or bad experience with breastfeeding. Suggest they become Baby-Friendlyo. You'll be helping other moms!

If you have questions, persistent pain, or can't hear swallowing, ask for help right away!

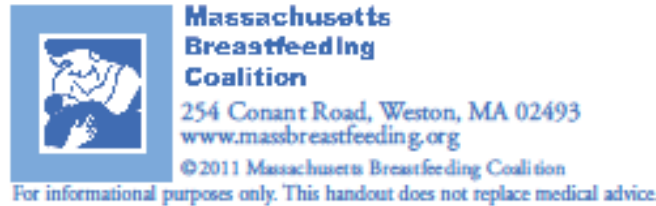


Show handout 3 (3-6 months) and go over it ( 5-7 minutes)

3 to 6 Months

\section{Your Baby's Development}

This time is all about parents and bables falling in love. Most bables are eating and sleeping more regularly. They are also responding more actively to parents and caregivers. Over the next few months, you will begin learning about your baby's preferences-what he likes and dislikes, how she prefers to sleep, eat and play. What are you leaming about your little one?

\section{What Your Baby Can Do}

I am learning to control my body.

- I push myself up to see the people I love and the things that interest me. I roll to try to get closer to you or to an interesting toy or object.

- I can sit with help and hold my head steady.

- I may start to rock back and forth on my hands and knees to get ready to crawl so I can get moving and explore.

I use my hands and fingers to explore.

- I reach for and grasp objects and toys.

I explore them with my fingers, hands, and mouth to figure out what they can do.

I communicate by using sounds, actions, and facial expressions.

-When you shake my rattle, I may smile and move my arms and legss to let you know I want to keep playing.

- I can make a few different sounds in response to your sounds-babbles, coos, and gurgles.

\section{What You Can Do}

Place your baby in different positions to help her develop new skills like rolling, creeping, and crawling.

- Make sure she gets time to play on both her back and stomach.

- Help her sit with support. This allows her to explore in new ways.

- Be sure she is always put to sleep on her back.

Offer your baby toys to explore that have different shapes, sizes, textures, and sounds. Show him ways to use these objects by shaking, banging, pushing, and dropping.

Watch and respond to your baby's signals. You are smiling-I think you like looking in the mirror. Do you want to look at yourself again?

Have back-and-forth "conversations" with your baby. When you reply to her babbles, she knows you care about what she is saying. This helps her learn to talk.
I am getting used to the world around me. - I may be starting to develop a more regular eating and sleeping schedule.

- I am beginning to notice daily routines. When you turn the lights down, I am learning it is time for sleep.
Create routines for your baby.

- Help him learn it's time for sleep by doing the same things in the same order each night, such as bath, books, feeding, and then a lullaby.

- Make up a song that you sing as you are getting ready to feed your baby. Each time he hears it, he'll know milk is coming. This may calm him and also help him learn to wait.

As you use this resource, remember that your child may develop skills faster or slower than indicated here and still be growing just fine. Talk with your child's health care prowider or other trusted professional if you have questions. 
Show handout 4 (6-9 months) and go over it ( 5-7 minutes)

\section{6 to 9 Months}

\section{Your Baby's Development}

This is a time of great fun for parents as they watch their babies become eager explorers who are thrilled to discover that they can make things happen. A 7-month-old knows, When I smille, mommy smiles back! A 9-month-old lifts her arms to tell her dad, I want you to pick me up. How is your baby making things happen?

\section{What Your Baby Can Do}

I am learning to think and solve problems.

-When a toy drops to the floor, I look to see where it went.

- I figure out how things work by copying what I see you and others do.

I can control my body.

- I can pick up small objects using my thumb and other fingers.

- I can sit on my own, which helps me explore in new ways.

- I may crawl or scoot to get around. I might even pull up on furniture to stand.

I am working hard to communicate with you.

- I babble a lot. When someone talks to me,

I make sounds back.

- I use my voice to express feelings, like joy and anger.

- I copy actions you make, like waving "byebye" and shaking my head "no-no."

\section{What You Can Do}

Comment on what your baby does to make things happen. You used your voice to let me know you wanted me to keep playing peek-boo. Let your baby explore interesting objectslike toys with buttons to push.

Begin letting your child practice picking up babysafe foods like slices of banana, if you'd like your child to learn to feed himself. Give your baby time to move around on his own. This builds muscle strength and coordination.
My personality is starting to show.

- I may love to meet new people or need time to feel comfortable with someone I don't know yet.

- I may like lots of sound and activity or I may prefer thingss to be more quiet and calm.

- I may be very active or more interested in watching.
Use words to describe your baby's feelings: You are mad that Daddy took away the crayon. You can chew on this rattle instead.

If your baby is looking at something, point at it and explain: That's a radio. It plays music.

Copy your baby's sounds and actions. If she waves, wave back and say Hello!

Notice how your baby likes to play and explore. Does she like to move or does she prefer to sit and watch the world around her?

See how your baby reacts to sounds, sights, and social activity. What does she seem to enjoy? What does she seem to dislike or get over whelmed by?

As you use this resource, remember that your child may develop skills faster or slower than indicated here and still be growing just fine. Talk with your child's health care prowider or other trusted professional if you have questions. 
Show handout 5 (9-12 months) and go over it ( 5-7 minutes)

\section{9 to 12 Months \\ Your Baby's Development}

Bables are becoming good communicators as they get closer to turning 1 year old. This makes it a delightful time for parents. Babies can use their actions and sounds to let loved ones know what they want, like handing a book to a parent so that she'll read it aloud. How does your baby "tell" you what he wants?

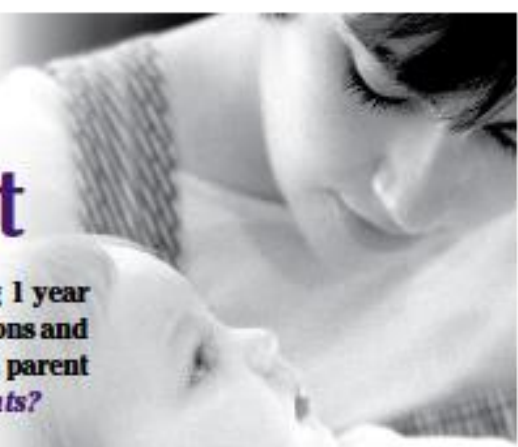

\section{What Your Baby Can Do}

I can understand more words than I can say.

- I am starting to understand what you say to me.

I can even follow simple directions like Go get the ball

- I tell you what I want with my sounds and body movements. I may say a word or two, like mama.

I can creep and crawl.

- I have found my own way of crawling -on my hands or knees, on my stomach, "crab crawling" by moving backwards and sideways, or even scooting on my bottom!

- I walk while holding on to furniture or your hand. I may even start walking on my own.

I know that things still exist even though

I can't see them-especially you!

- I may cry when you leave because I know you are still out there somewhere and I want you to come back!

\section{I love to do things over and over again.}

- This is how 1 practice and figure out how things work.

- Repetition also helps build my memory.

\section{What You Can Do}

Tell your baby what is happening and what you will do next: After your milk, it is time for a nap. This helps her learn language. Routines also let her know what to expect.

Put your baby's sounds and actions into words. You are pushing your food away. I think you are telling me you are all done.

Name things your baby looks at or points to: That's the moon. The moon comes out at night.

Give your baby lots of time and a safe place to practice new skills like crawling and walking.

Make a "trail of toys" in a childsafe place in your house. Line up several interesting objects (a wooden spoon, a plastic bowl, a brightly colored dishcloth) that your child can crawl to and explore.

Play hide-andseek games. This helps your baby learn that things that disappear also reappear.

Be sure to say good-bye to your baby. Never sneak out. This builds his trust in you and helps him learn to deal with difficult feelings.

Help your child take the next step in her play. If she is banging two blocks together, see if she'd like to try stacking them.

Offer your child a ball to toss, a rattle to shake, or a scarf to swing. These activities help children learn how thingss work. They also build the muscles in their hands that will help them learn to write. 
12 to 15 Months

\section{Your Child's Development}

This is a really fun time for parents, as 1-year-olds are able to explore the world in new ways and are eager to do things all by myself? They watch their loved ones very carefully and copy a lot of what they see. This is one of the most important ways toddlers learn how the world works. How do you see your chlld learning from watching you?

\section{What Your Toddler Can Do}

I'm moving!

- I may walk on my own or by holding your hand.

- I am learning to crawl up stairs but can't come down yet.

- I can throw a ball and turn pages in books.

I'm starting to talk and understand so much more.

- I may use some words like duhduh for dog-

- I can show you what I want through my actions. I may bang my high chair when I want more food.

- If you ask me to, I can point to a body part or a picture in a book.

\section{What You Can Do}

Give your child just enough help to reach his goal. If he wants to stand, let him hold your fingers for balance.

Support your child as he practices new skills like climbing stairs. Children need time to work on these new skills...safely!

Encourage your child to turn the pages when you read together.

Choose books about things that interest your child like animals or other children.

Build your child's vocabulary. If she points to or says bus, you can say: The school bus is driving down the street.

Name the people, places, and things that your child sees each day: That's a garbage truck taking our trash.

Play games that involve following directions: Throw the ball to me.

Involve your child in self-help tasks like washing his hands.

Follow your child's lead. Let him choose what toys or games to play.

I say no or show you in other ways that I want to do things on my own.

I love to imitate.

- I copy actions lve seen other people do, like stir a pot or talk on the phone.

Join in your child's play. If you see her putting a blanket on her toy bear, ask: Does Teddy need a bottle before bed? Give her objects to play with that she sees in "real life," like plastic dishes, a toy telephone, a small dust broom.

As you use this resourve, remember that your child may develop skills faster or slower than indicated here and still be growing just fine. Talk with your child's health care prowider or other trusted professional if you have questions. 


\section{Appendix T}

Research Participant Receipt

TITLE OF STUDY: WVU Health \& Infant Feeding Study

PRINCIPAL INVESTIGATOR: Sarah E. Hayes, $M S, M P H$

PARTICIPANT NAME:

PARTICIPANT SIGNATURE:

DATE:

Participant Permanent Address:

Participant Preferred Mailing Address:

$\square$ Same as above

OFFICE USE ONLY

RESEARCH STAFF SIGNATURE:

GIFT CARD \# _ _ GIFT CARD AMOUNT: \$ 


\section{Appendix U}

\section{Postpartum Phone Call Planning Form}

Phone 1:

Type:

Phone 2:

Type:

Phone 3:

Type:

Best times to call:

Can we leave a voicemail message? Yes

No

Can we leave a message with a family member/friend? Yes

No

Email address 1:

Email address 2:

Other methods by which we have permission to contact you? (e.g., social media)

Anything else we should know about contacting you?

\section{OFFICE USE ONLY}

Participant ID:

Due date:
Date of prenatal visit:

Target call date: 


\section{Appendix V}

\section{Email from Participant}

\section{Hi Sarah!}

Hope all is well! I exclusively nursed for 11.5 weeks before I had to fly out of town! I think this is due to our conversation! Thank you for helping me! I don't know if that was the projects [sic] intent or not but thank you! 


\section{Appendix W}

Supplementary Tables

Table A1

Frequency (and \%) of Participant Prenatal and Postpartum Medications or Medication Type

\begin{tabular}{lcccccc}
\hline & \multicolumn{3}{c}{ Prenatal } & \multicolumn{3}{c}{ Postpartum } \\
\hline & Overall & MI & PE & Overall & MI & PE \\
& $(n=81)$ & $(n=41)$ & $(n=40)$ & $(n=79)$ & $(n=40)$ & $(n=39)$ \\
\hline Allergy & $6(7.4)$ & $4(9.8)$ & $2(5.0)$ & $3(3.8)$ & $3(7.5)$ & - \\
Antacids & $4(4.9)$ & $2(4.9)$ & $2(5.0)$ & $1(1.3)$ & - & $1(2.6)$ \\
Antibiotics & $2(2.8)$ & $2(4.9)$ & - & $3(3.8)$ & $1(2.5)$ & $2(5.1)$ \\
Anti-depressant & $3(3.7)$ & $1(2.4)$ & $2(5.0)$ & $5(6.3)$ & $2(5.0)$ & $3(7.7)$ \\
Anti-nausea & $7(8.6)$ & $3(7.3)$ & $4(10.0)$ & - & - & - \\
Birth control & - & - & - & $4(5.1)$ & $2(5.0)$ & $2(5.1)$ \\
Blood pressure & - & - & - & $2(2.5)$ & $1(2.5)$ & $1(2.6)$ \\
Buprenorphine & $2(2.5)$ & - & $2(5.0)$ & $2(2.5)$ & $1(2.5)$ & $1(2.6)$ \\
Buspirone & $1(1.2)$ & $1(2.4)$ & - & - & - & - \\
Diabetes & $4(4.9)$ & $3(7.3)$ & $1(2.5)$ & - & - & - \\
Heparin & - & $1(2.4)$ & - & - & - & - \\
Levothyroxine & $5(6.7)$ & $2(4.9)$ & $3(7.5)$ & $1(1.3)$ & - & $1(2.6)$ \\
OTC pain reliever & $3(3.7)$ & $2(4.9)$ & $1(2.5)$ & $4(5.1)$ & $2(5.0)$ & $2(5.1)$ \\
Stool softener & $3(3.7)$ & - & $3(7.5)$ & $3(3.8)$ & $2(5.0)$ & $1(2.6)$ \\
Triptans & - & - & - & $1(1.3)$ & - & $1(2.6)$ \\
\hline
\end{tabular}

Note $. \mathrm{MI}=$ Motivational Interviewing $; \mathrm{PE}=$ psychoeducation. Medications listed here do not include prenatal vitamins; OTC $=$ over the counter. 
Table A2

Frequency (and \%) of History of Medical Conditions Reported by Participant

\begin{tabular}{lccc}
\hline & $\begin{array}{c}\text { Overall } \\
(n=81)\end{array}$ & $\begin{array}{c}\text { MI } \\
(n=41)\end{array}$ & $\begin{array}{c}\text { PE } \\
(n=40)\end{array}$ \\
\hline Anemia & $3(3.7)$ & $2(4.9)$ & $1(2.5)$ \\
Asthma & $1(3.7)$ & - & $1(2.5)$ \\
Cardiovascular problem & $4(4.9)$ & $1(2.4)$ & $3(7.5)$ \\
Chronic sinusitis & $1(3.7)$ & $1(2.4)$ & - \\
Cleft palate & $1(3.7)$ & $1(2.4)$ & - \\
Concussion & $1(3.7)$ & - & $1(2.5)$ \\
Herpes & $1(3.7)$ & - & $1(2.5)$ \\
Hypertension & $3(3.7)$ & $2(4.9)$ & $1(2.5)$ \\
Migraine & $3(3.7)$ & $2(4.9)$ & $1(2.5)$ \\
Musculoskeletal problem & $3(3.7)$ & $1(2.4)$ & $2(5.0)$ \\
Polycystic ovarian syndrome & $2(2.5)$ & - & $2(5.0)$ \\
Thyroid disorder & $6(7.4)$ & $4(9.8)$ & $2(5.0)$ \\
\hline
\end{tabular}

Note. $\mathrm{MI}=$ Motivational Interviewing; $\mathrm{PE}=$ psychoeducation. 
Table A3

Frequency (and \%) of Participant Pregnancy Complications at Baseline

\begin{tabular}{llcc}
\hline & $\begin{array}{c}\text { Overall } \\
(n=81)\end{array}$ & $\begin{array}{c}\text { MI } \\
(n=41)\end{array}$ & $\begin{array}{c}\text { PE } \\
(n=40)\end{array}$ \\
\hline Placenta previa & $2(2.5)$ & - & $2(5.0)$ \\
Low amniotic fluid & $1(3.7)$ & - & $1(2.5)$ \\
Severe cramping & $1(3.7)$ & - & $1(2.5)$ \\
Preterm labor & $1(3.7)$ & - & $1(2.5)$ \\
Breech position & $1(3.7)$ & - & $1(2.5)$ \\
Low progesterone & $1(3.7)$ & - & $1(2.5)$ \\
Hemorrhaging & $2(2.5)$ & $1(2.4)$ & $1(2.5)$ \\
Shortening of cervix & $1(3.7)$ & $1(2.4)$ & - \\
Sciatica & $1(3.7)$ & $1(2.4)$ & - \\
\hline
\end{tabular}

Note. $\mathrm{MI}=$ Motivational Interviewing; $\mathrm{PE}=$ psychoeducation. 